\title{
The World Federation of Societies of Biological Psychiatry (WFSBP) Guidelines for the Biological Treatment of Bipolar Disorders: Acute and Long-term Treatment of Mixed states in Bipolar Disorder
}

Heinz Grunze ${ }^{1,2 *}$, Eduard Vieta ${ }^{3}$, Guy M. Goodwin ${ }^{4}$, Charles Bowden ${ }^{5}$, Rasmus W. Licht ${ }^{6,7}$ Jean-Michel Azorin ${ }^{8}$, Hans-Jürgen Möller ${ }^{9}$, Siegfried Kasper ${ }^{10}$, WFSBP Task Force on Treatment Guidelines for Bipolar Disorders ${ }^{11}$

1 Newcastle University, Institute of Neuroscience, Newcastle upon Tyne, United Kingdom

2 Paracelsus Medical University, Nuremberg, Germany

3 Bipolar Disorders Programme, Institute of Neuroscience, Hospital Clinic, University of Barcelona, IDIBAPS, CIBERSAM, Barcelona, Catalonia, Spain

4 University of Oxford, Department of Psychiatry, Warneford Hospital, Oxford, United Kingdom

5 University of Texas Health Science Center, Dept. of Psychiatry, San Antonio, USA

6 Psychiatric Research Unit, Aalborg University Hospital, Psychiatry, Aalborg, Denmark

7 Clinical Department of Medicine, Aalborg University, Aalborg, Denmark

8 Hospital Ste. Marguerite, Marseille, France.

9 Ludwigs-Maximilian University, Department of Psychiatry and Psychotherapy, Munich, Germany

10 Medical University of Vienna, Department of Psychiatry and Psychotherapy, Vienna, Austria

11 WFSBP Task Force on Treatment Guidelines for Bipolar Disorders:

Siegfried Kasper (Chairman, Austria), Guy Goodwin (Co-Chairman, United Kingdom), Charles Bowden (Co-Chairman, USA), Heinz Grunze (Secretary, United Kingdom), HansJürgen Möller (WFSBP Past-President, Germany), Rasmus W. Licht (Denmark), Eduard Vieta (Spain). 
Hagop Akiskal (USA), José Luis Ayuso-Gutierrez (Spain), Jean-Michel Azorin (France), Michael Bauer (Germany), Per Bech (Denmark), Michael Berk (Australia), Istvan Bitter (Hungary), Graham Burrows (Australia), Joseph Calabrese (USA), Giovanni Cassano (Italy), Marcelo Cetkovich-Bakmas (Argentina), John C. Cookson (United Kingdom), I. Nicol Ferrier (United Kingdom), Maria Luisa Figueira (Portugal), Wagner F. Gattaz (Brazil), Frederik K. Goodwin (USA), Gerhard Heinze (Mexico), Chantal Henry (France), Teruhiko Higuchi (Japan), Robert M. Hirschfeld (USA), Cyril Höschl (Czech Republik), Edith Holsboer-Trachsler (Switzerland), Kay Redfield Jamison (USA), Cornelius Katona (UK), Martin Keller (USA), Lars Vedel Kessing (Denmark), E. Kostukova (Russia), Hever Kruger (Peru), Parmanand Kulhara (India), Veronica Larach (Chile), Veronica Larach (Chile), Odd Lingjaerde (Norway), Henrik Lublin (Denmark), Mario Maj (Italy), Julien Mendlewicz (Belgium), Roberto Miranda Camacho (Mexico), Philip Mitchell (Australia), S. Mosolov (Russia), Stuart Montgomery (United Kingdom), Charles Nemeroff (USA), Willem Nolen (The Netherlands), Timucin Oral (Turkey), Eugene S. Paykel (United Kingdom), Robert M.

Post (USA), Stanislaw Puzynski (Poland), Zoltan Rihmer (Hungary), Janusz K. Rybakowski (Poland),Simavi Vahip (Turkey), Per Vestergaard (Denmark), Peter C. Whybrow (USA), Kazuo Yamada (Japan), Lakshmi Yatham (Canada)

\section{Correspondence:}

Prof. Dr. Heinz Grunze

Institute of Neuroscience

Department of Psychiatry, RVI

Newcastle University

Newcastle upon Tyne NE1 4LP, United Kingdom

Tel: +44 1912825765 Fax: +44191222 6162

E-mail: Heinz.Grunze@ncl.ac.uk

Key words: mixed states, bipolar disorder, pharmacotherapy, physical treatment, guideline 


\section{Acknowledgements}

We would like to specially thank Mrs Monica Gratacos for her great support in the initial literature search.

\section{Financial Disclosures of principal authors}

Heinz Grunze received grants/ research support, consulting fees and honoraria within the last three years from BMS, Gedeon Richter, Genericon, Janssen-Cilag, Lundbeck, Otsuka, Pfizer, Servier.

Eduard Vieta received grants/research support, consulting fees and honoraria within the last three years from Ab-Biotics, Allergan, Angelini, AstraZeneca, Bristol-Myers Squibb, Dainippon Sumitomo Pharma, Eli Lilly, Farmindustria, Ferrer, Forest Research Institute, Gedeon Richter, Glaxo-Smith-Kline, Janssen, Johnson \& Johnson, Lundbeck, Otsuka, Pfizer Inc, Sanofi-Aventis, Servier, Sunovion, Takeda, and Tecnifarma.

Siegfried Kasper received grants/research support, consulting fees and/or honoraria within the last three years from Angelini, AOP Orphan Pharmaceuticals AG, AstraZeneca, Eli Lilly, Janssen, KRKA-Pharma, Lundbeck, Neuraxpharm, Pfizer, Pierre Fabre, Schwabe and Servier.

Guy Goodwin is a NIHR Senior Investigator, holds a grant from Wellcome Trust, holds shares in P1vital and has served as consultant, advisor or CME speaker for Angelini, Compass pathways, MSD, Lundbeck (/Otsuka or /Takeda), Medscape, P1Vital, Pfizer, Servier and Shire. The views expressed are those of the author(s) and not necessarily those of the NHS, the NIHR or the Department of Health.

Charles Bowden has no conflicts of interest to report.

Rasmus W. Licht received research grants, consulting fees and honoraria within the last three years from Bristol-Myers Squibb, Janssen Cilag, Lundbeck, Otsuka, Servier and Sunovion

Jean-Michel Azorin received grants/research support, consulting fees and honoraria within the last three years from Allergan, AstraZeneca, Janssen Cilag, Lundbeck, Otsuka, Servier, Takeda and Teva.

Hans-Jürgen Möller received grant/research support, consulting fees and honoraria within the last years from Eli Lilly, Lundbeck, Servier and Schwabe 


\section{Abbreviations}

AE Adverse event

AED Antiepileptic drug

AIMS Abnormal Involuntary Movement Scale

BAP British Association for Psychopharmacology

BARS Barnes Akathisia Rating Scale

BD-I Bipolar I disorder

BD-II Bipolar II disorder

CANMAT Canadian Network for Mood and Anxiety Treatments

CDRS-R Children’s Depression Rating Scale, Revised

CBT Cognitive behavioural therapy

CE Category of evidence this was CE in the prior guidelines

CGI-BP Clinical Global Impression- Bipolar

CI Confidence interval

DSM Diagnostic and Statistical Manual

DBS Deep brain stimulation

ECT Electroconvulsive therapy

eGFR Estimated glomerular filtration rate

EMS Extrapyramidal motor symptoms (EPS, for extrapyramidal symptoms, is more commonly used in the US.

ER $\quad$ Extended release

ESRS Extrapyramidal Symptoms Rating Scale

FE $\quad$ Further evidence

FDA US Food and Drug administration 
HAM-D Hamilton Rating Scale for Depression

HR Hazard ratio

ICD International Classification of Diseases

IDS Inventory of Depressive Symptoms

IDS-SR Inventory of Depressive Symptoms

ISBD International Society for Bipolar Disorder

ITT Intend-to-treat

KM Kaplan Meier

LAI Long acting injectable

LOCF Last observation carried forward

MADRS Montgomery-Asberg Depression Rating Scale

MDE Major depressive episode

MMRM Mixed effect Model Repeat Measurement

MOAT Multistate Outcome Analysis of Treatments in Bipolar Disorder

MS Mood stabilizer

NNT Numbers needed to treat

OFC Olanzapine-Fluoxetine combination

OR Odds ratio

RC Rapid cycling

RCT Randomized controlled trial

RG Recommendation grade

RR Relative risk

rTMS Repetitive transcranial magnetic stimulation

SAS Simpson-Angus extrapyramidal side effect scale 
SFBN Stanley Foundation Bipolar Network

ST $\quad$ Safety \& Tolerability

STEP-BD Systematic Treatment Enhancement Program for Bipolar Disorder

TAU Treatment as usual

TEAS Treatment emergent affective switch

OR Odds ratio

TEE Treatment emergent episode

VNS Vagus nerve stimulation

WFSBP World Federation of Societies of Biological Psychiatry

YMRS Young Mania Rating Scale

\section{Contents}

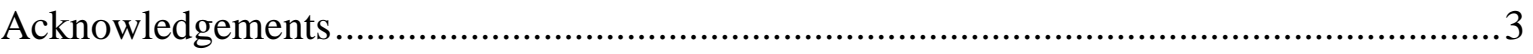

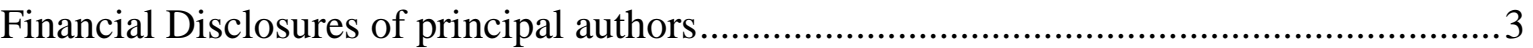

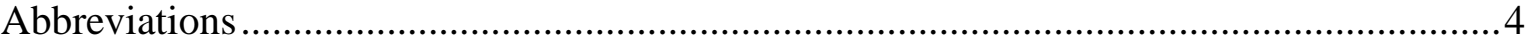

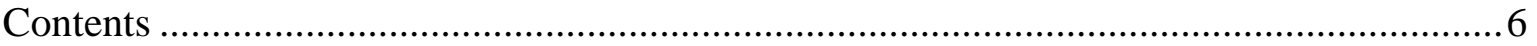

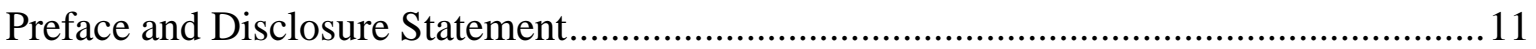

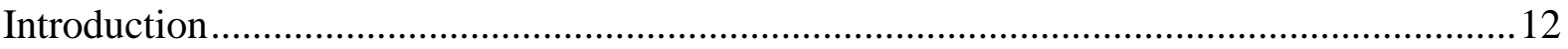

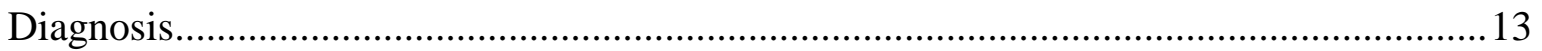

Epidemiology and clinical relevance of mixed states.....................................................17

Continuation and maintenance treatment after an acute mixed episode..............................20

Prevention of mixed episodes after a manic or depressive index episode..........................21

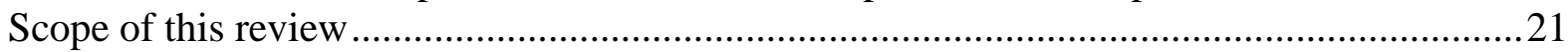

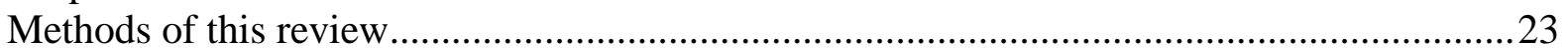

Medications commonly used in mixed states and their ranking by evidence .........................22

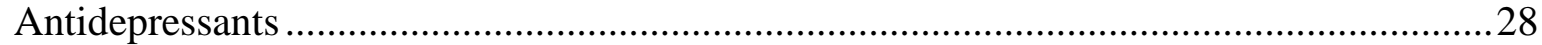

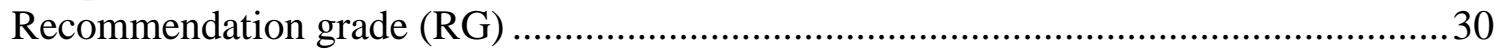




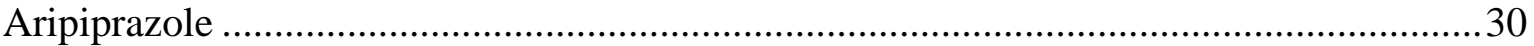

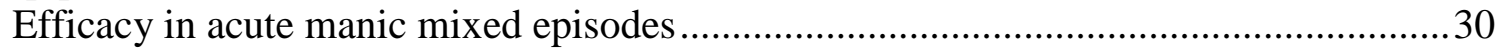

Efficacy in acute depressive mixed episodes............................................................

Efficacy in maintenance treatment after an acute mixed episode in preventing episodes

of any polarity or a new manic, depressive, or mixed episode ........................................32

Efficacy in maintenance treatment after an acute manic or depressed episode in

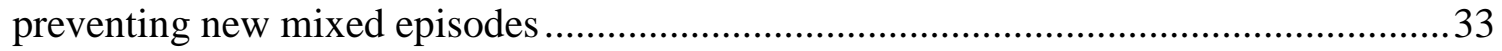

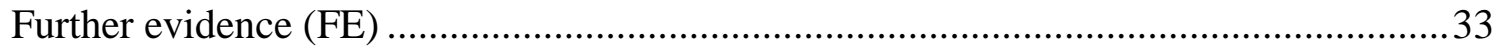

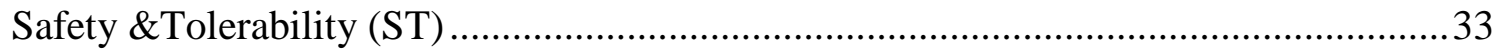

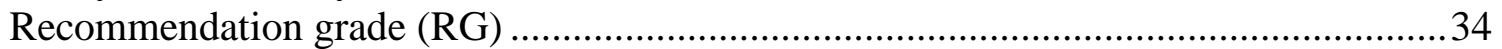

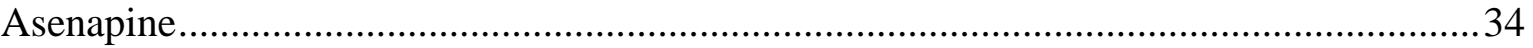

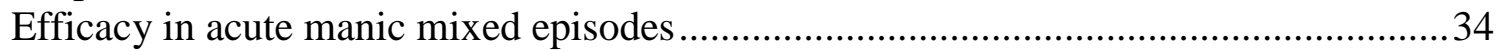

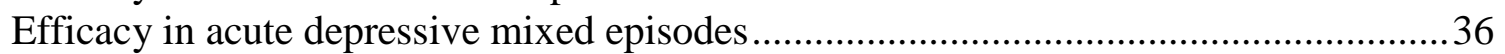

Efficacy in maintenance treatment after an acute mixed episode in preventing episodes

of any polarity or a new manic, depressive, or mixed episode ........................................36

Efficacy in maintenance treatment after an acute manic or depressed episode in

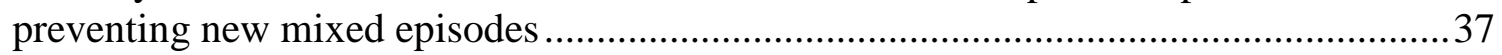

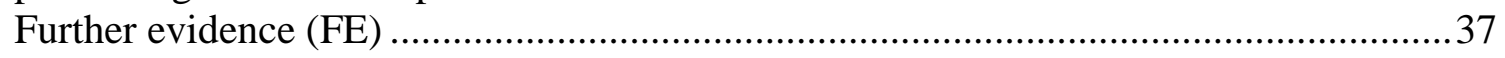

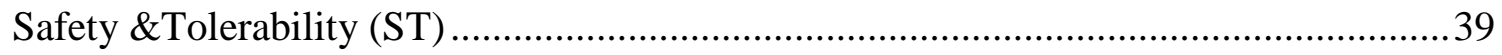

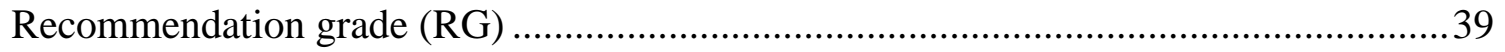

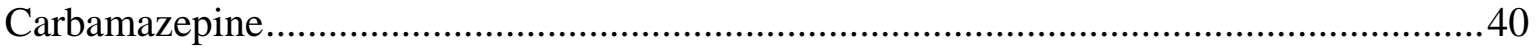

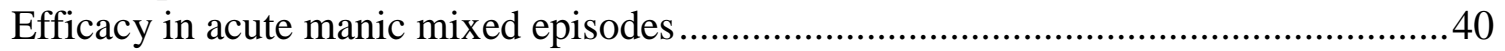

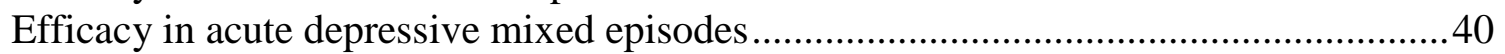

Efficacy in maintenance treatment after an acute mixed episode in preventing episodes

of any polarity or a new manic, depressive, or mixed episode .......................................4 41

Efficacy in maintenance treatment after an acute manic or depressed episode in

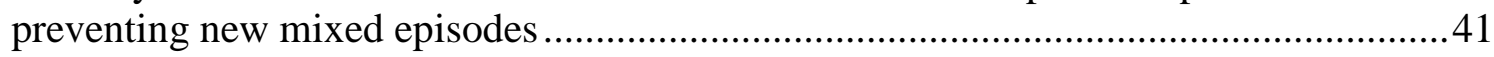

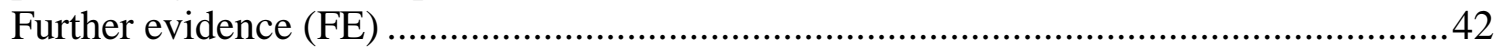

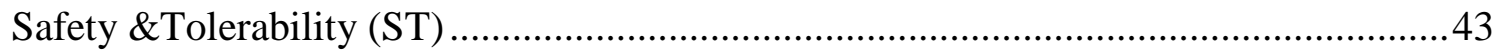

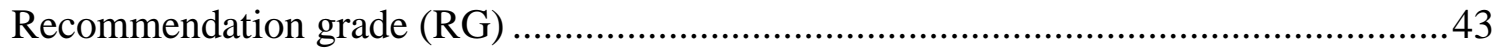

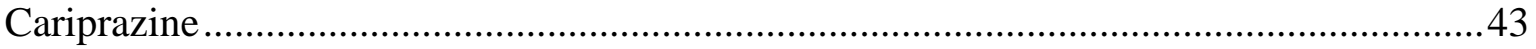

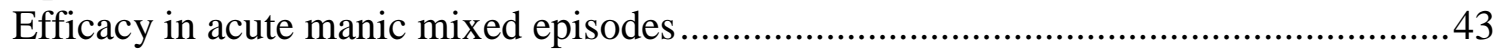

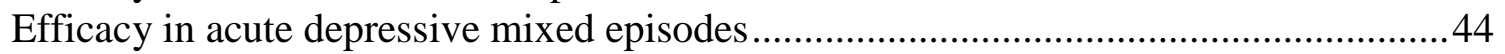

Efficacy in maintenance treatment after an acute mixed episode in preventing episodes

of any polarity or a new manic, depressive, or mixed episode ........................................44

Efficacy in maintenance treatment after an acute manic or depressed episode in

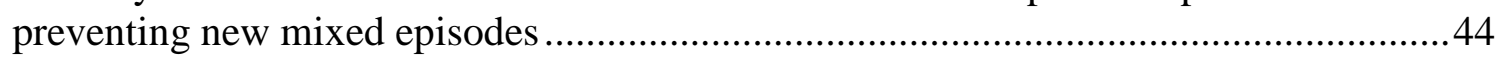

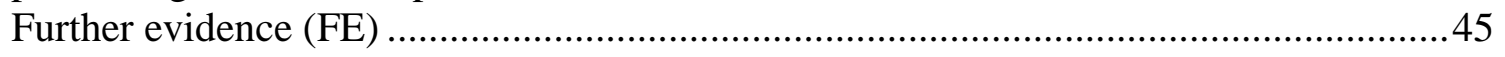

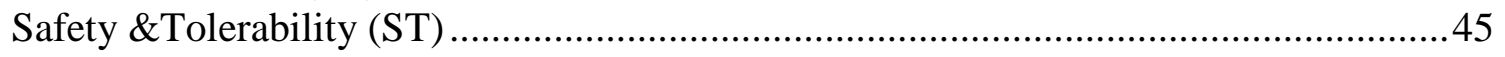

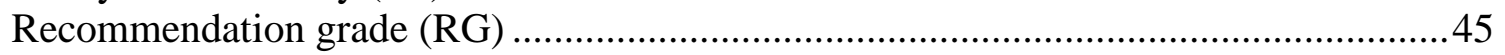

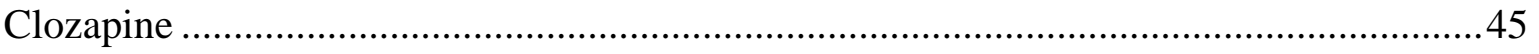

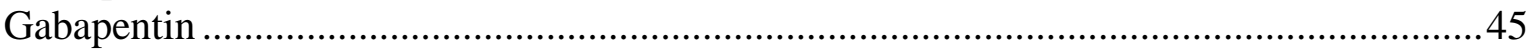

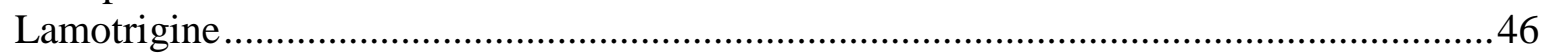

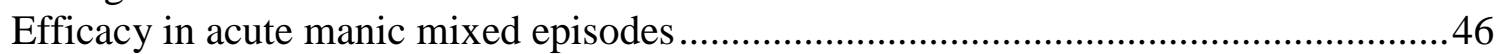

Efficacy in acute depressive mixed episodes..............................................................46

Efficacy in maintenance treatment after an acute mixed episode in preventing episodes

of any polarity or a new manic, depressive, or mixed episode ........................................46

Efficacy in maintenance treatment after an acute manic or depressed episode in

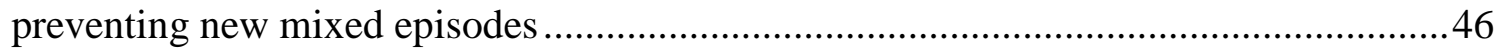

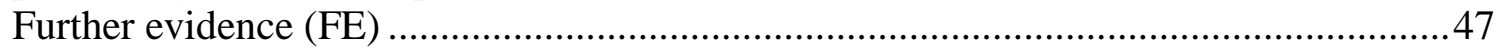


Safety \&Tolerability (ST) ....................................................................................4

Recommendation grade (RG) ................................................................................

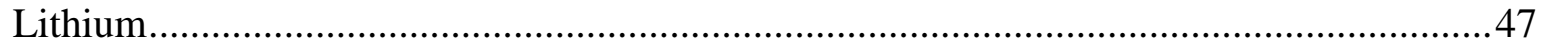

Efficacy in acute manic mixed episodes .........................................................................4

Efficacy in acute depressive mixed episodes.............................................................4

Efficacy in maintenance treatment after an acute mixed episode in preventing episodes

of any polarity or a new manic, depressive, or mixed episode .........................................48

Efficacy in maintenance treatment after an acute manic or depressed episode in

preventing new mixed episodes ................................................................................4

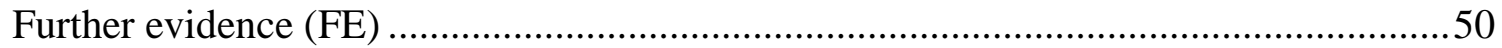

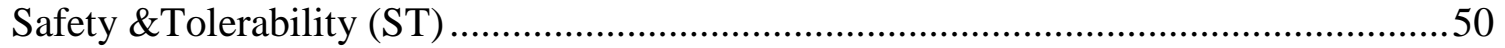

Recommendation grade (RG) ……………………............................................. 51

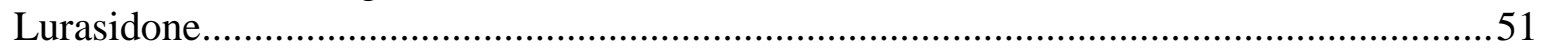

Efficacy in acute manic mixed episodes ......................................................................51

Efficacy in acute depressive mixed episodes.................................................................51

Efficacy in maintenance treatment after an acute mixed episode in preventing episodes

of any polarity or a new manic, depressive, or mixed episode ........................................52

Efficacy in maintenance treatment after an acute manic or depressed episode in

preventing new mixed episodes ................................................................................52

Further evidence (FE) …………………………………….................................53

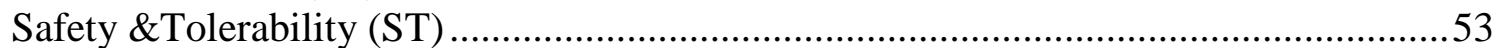

Recommendation grade (RG) ………………………….....................................53

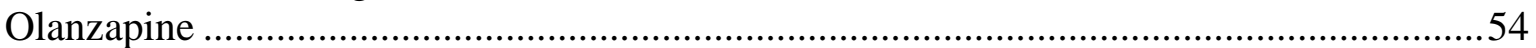

Efficacy in acute manic mixed episodes....................................................................54

Efficacy in acute depressive mixed episodes..................................................................56

Efficacy in maintenance treatment after an acute mixed episode in preventing episodes

of any polarity or a new manic, depressive, or mixed episode .........................................56

Efficacy in maintenance treatment after an acute manic or depressed episode in

preventing new mixed episodes .................................................................................57

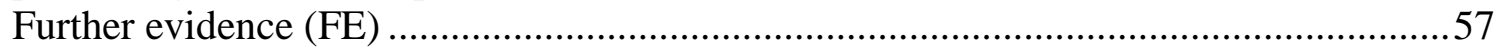

Safety \&Tolerability (ST) ……………………………….................................5

Recommendation grade (RG) …………………………....................................59

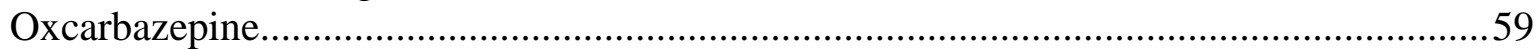

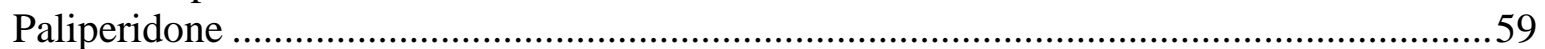

Efficacy in acute manic mixed episodes ........................................................................59

Efficacy in acute depressive mixed episodes..............................................................60

Efficacy in maintenance treatment after an acute mixed episode in preventing episodes

of any polarity or a new manic, depressive, or mixed episode .........................................6

Efficacy in maintenance treatment after an acute manic or depressed episode in

preventing new mixed episodes ...................................................................................6

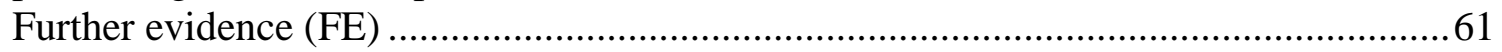

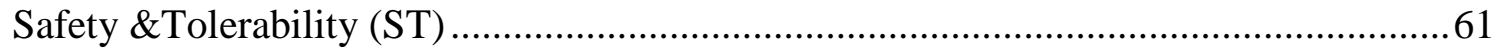

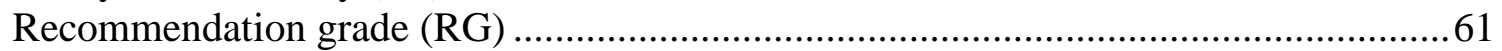

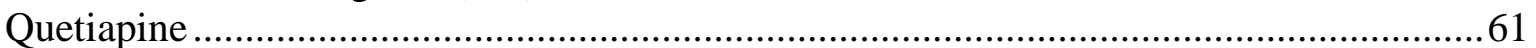

Efficacy in acute manic mixed episodes .......................................................................61

Efficacy in acute depressive mixed episodes..................................................................62

Efficacy in maintenance treatment after an acute mixed episode in preventing episodes

of any polarity or a new manic, depressive, or mixed episode ..........................................62

Efficacy in maintenance treatment after an acute manic or depressed episode in preventing new mixed episodes 
Further evidence (FE) …………………............................................................63

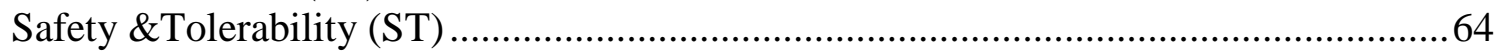

Recommendation grade (RG) .............................................................................64

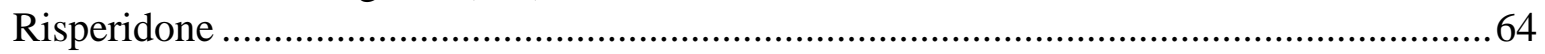

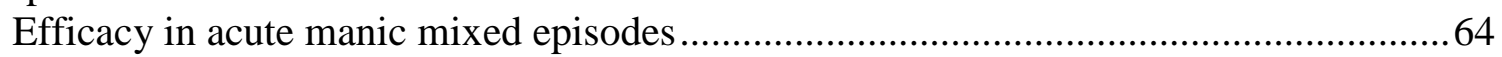

Efficacy in acute depressive mixed episodes.................................................................65

Efficacy in maintenance treatment after an acute mixed episode in preventing episodes

of any polarity or a new manic, depressive, or mixed episode .........................................65

Efficacy in maintenance treatment after an acute manic or depressed episode in preventing new mixed episodes ................................................................................66

Further evidence (FE) …………………………………………………………...66

Safety \&Tolerability (ST) ...................................................................................6

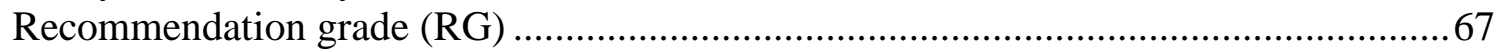

Typical antipsychotics (first generation antipsychotics)......................................................67

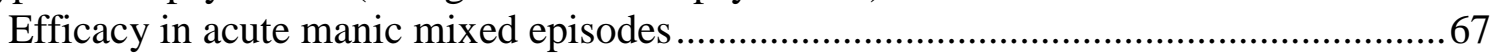

Efficacy in acute depressive mixed episodes.................................................................67

Efficacy in maintenance treatment after an acute mixed episode in preventing episodes

of any polarity or a new manic, depressive, or mixed episode ........................................67

Efficacy in maintenance treatment after an acute manic or depressed episode in

preventing new mixed episodes ....................................................................................68

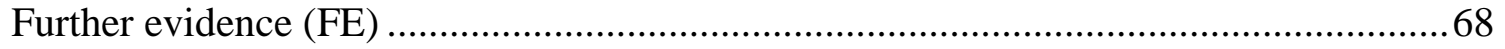

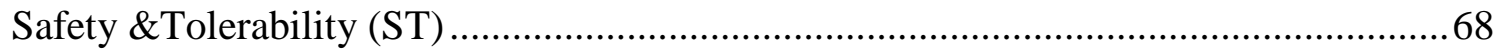

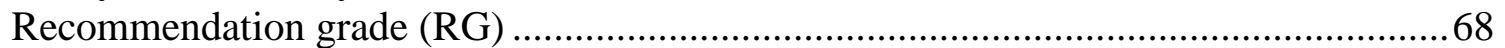

Valproate (incl. Divalproate, Divalproex, Valpromide) .....................................................69

Efficacy in acute manic mixed episodes.......................................................................69

Efficacy in acute depressive mixed episodes...................................................................69

Efficacy in maintenance treatment after an acute mixed episode in preventing episodes

of any polarity or a new manic, depressive, or mixed episode .........................................70

Efficacy in maintenance treatment after an acute manic or depressed episode in preventing new mixed episodes .................................................................................70

Further evidence (FE) …………………………………...................................

Safety \&Tolerability (ST) …………………………………………………….....71

Recommendation grade (RG) ……………………………....................................

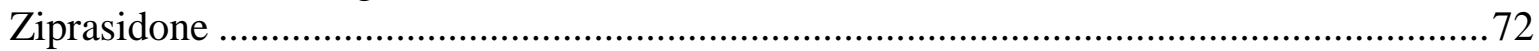

Efficacy in acute manic mixed episodes ........................................................................

Efficacy in acute depressive mixed episodes.................................................................... 73

Efficacy in maintenance treatment after an acute mixed episode in preventing episodes

of any polarity or a new manic, depressive, or mixed episode ..........................................73

Efficacy in maintenance treatment after an acute manic or depressed episode in

preventing new mixed episodes ..................................................................................

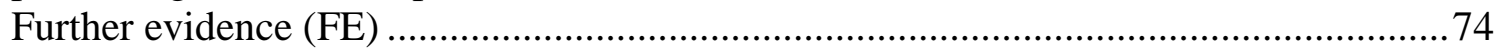

Safety \&Tolerability (ST) ……………………………………………………...74

Recommendation grade (RG) .............................................................................

Other Atypical Antipsychotics used in Bipolar Disorder .................................................75

Other Anticonvulsants used in Bipolar Disorder ...............................................................76

Other medication tested in bipolar mixed states .................................................................78

Electroconvulsive Therapy (ECT) …………………….........................................

Efficacy in acute manic mixed episodes .......................................................................78

Efficacy in acute depressive mixed episodes................................................................. 80

Efficacy in maintenance treatment after an acute mixed episode in preventing episodes 
of any polarity or a new manic, depressive, or mixed episode

Efficacy in maintenance treatment after an acute manic or depressed episode in

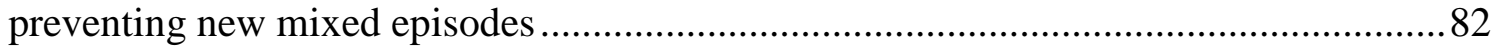

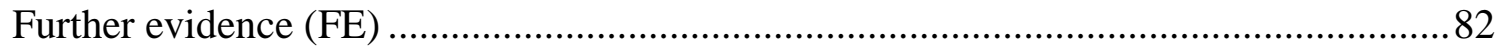

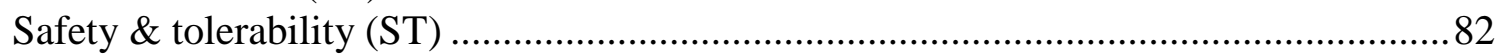

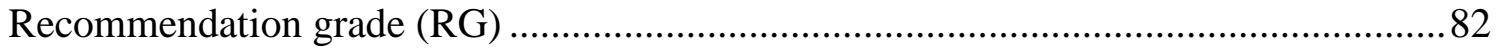

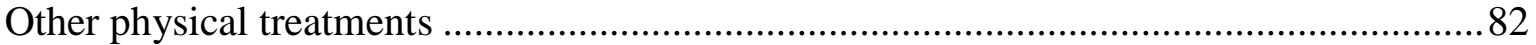

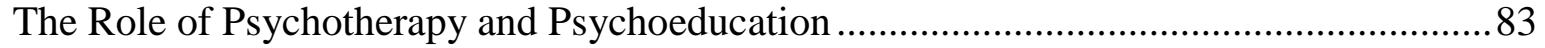

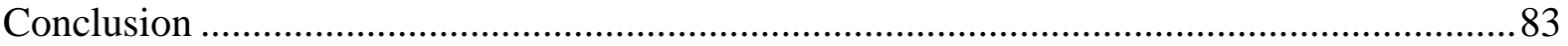

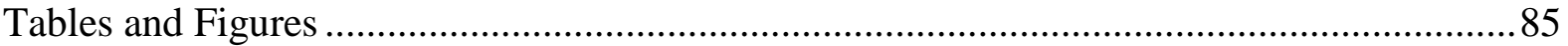

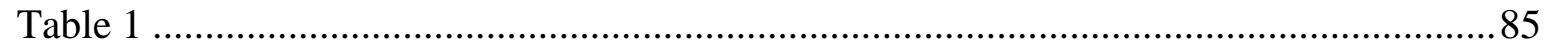

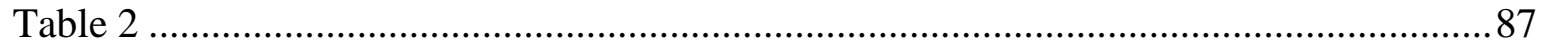

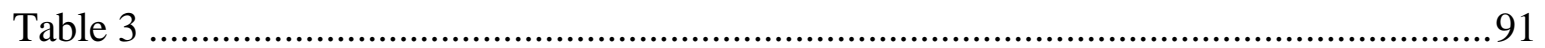

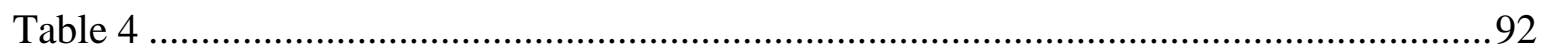

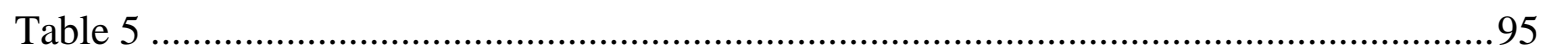

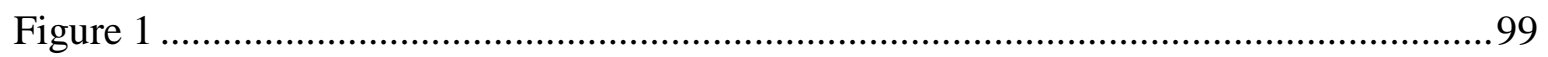

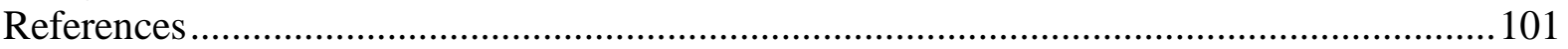




\section{Preface and Disclosure Statement}

This practice guideline for the biological, mainly pharmacological treatment and prevention of mixed episodes in bipolar disorder was developed by an international Task Force of the World Federation of Societies of Biological Psychiatry (WFSBP) and is part of a series covering the acute and maintenance treatment of bipolar disorder. The preparation of these guidelines has not been financially supported by any commercial organization.

This guideline has mainly been developed by psychiatrists and psychotherapists who are in active clinical practice. Experts of the task force were selected according to their expertise and with the aim to cover a multitude of different cultures.

In addition, some contributors are primarily involved in research or other academic endeavours. It is possible that through such activities some contributors have received income related to medicines discussed in this guideline. A number of mechanisms are in place to minimize the potential for producing biased recommendations due to conflicts of interest.

At the time of preparation of this manuscript the FDA licensing label "Mixed Episodes" relates to the narrow DSM-IV definition of mixed states, and has been granted to some medications as an extension of a license for the treatment of acute mania in Bipolar I disorder (BD-I). So far, no specific licensing by authorities exists for the DSM-5 specifier „with mixed features”, other mixed episodes subthreshold to DSM-IV definitions, mixed episodes in Bipolar II (BD-II), cyclothymia or Bipolar NOS disorder, or for the prevention of mixed episodes. Other authorities such as the EMA do not envisage a mixed episode label so far although they do now recognize the need (European Medicines Agency scientific commitee 2016). Consequently, recommendations in this guideline targeting those subtypes and symptom clusters in Bipolar disorder are often derived from data relating to mixed states in BD-I disorder, and not covered by a specific license („off-label use”). In addition, some drugs recommended in the present guideline may not be available in all countries, and approved doses may vary.

With the aim of having a comprehensive view of acute and maintenance treatment of mixed episodes in bipolar disorder, we reviewed published data on the efficacy of approved and non-approved pharmacological or physical therapies for mixed states, rated the evidence for their efficacy, and developed recommendations for specific symptom and treatment scenarios. 


\section{Introduction}

Although already well characterized by Weygandt (Weygandt 1899), a medical assistant of Kraepelin, mixed states only recently experienced the attention that they deserve, given their high frequency and unfavourable prognosis (Grunze and Walden 2005). The frequent occurrence of mixed states (up to $70 \%$ of manic patients simultaneously show symptoms of depression) underlines that $\mathrm{BD}$ is not a pure „folie à double forme“ (dual -form insanity) as it was originally coined by the French psychiatrist Jules Baillarger with mania and depression being distinct opposite poles (Baillarger 1854).

Although some information on the evidence for different treatments of mixed episodes had been included in the WFSBP mania guideline (Grunze et al. 2009) it appears timely to address mixed episodes in a guideline of its own. Traditionally, the evidence for response of mixed patients to pharmacological agents has been extrapolated from studies or trials that have enrolled both pure and mixed manic patients, assuming a comparable antimanic response to treatment for both subgroups of patients. Few of these reports, though, have examined whether there is differential efficacy in the subset of patients with mixed manic states. Mixed depression, i.e. depression with accompanying (hypo)manic symptoms, has received even much less attention than mixed mania, and therefore reliable data on its differential treatment response is even more scarce. The lack of available information results in the assumption in current clinical guidelines that, with exceptions, treatment of mixed presentations should be similar to pure manic states. However, clinical experience with the various tentative antimanic agents over recent years has suggested that a drug that is efficacious in one subtype of mania is not necessarily the treatment of choice for the other subtypes.

For depressive mixed episodes, the evidence for optimal treatment was too sparse at the time of release of the respective WFSBP guideline on the treatment of bipolar depression (Grunze et al. 2010) to justify specific treatment recommendations. In the meantime, this has changed to some degree, and clinicians may welcome some guidance on the pharmacological treatment of mixed depressive episodes. Finally, we now have some data on prophylactic treatment in patients with a mixed index episode that might be valuable to guide pharmacotherapy. 


\section{Diagnosis}

Bipolar disorder can be best conceptualised as a multiplex dysregulation syndrome involving besides mood a broad spectrum of basic mental qualities. The presence of depressive symptomatology during acute mania has been recognised for centuries and has been mentioned in 19th century German textbooks as mixtures („Mischungen” by Heinroth in 1818) or mid-forms („Mittelformen” by Griesinger in 1845) (Gonzalez-Pinto et al. 2007). In 1854, the French psychiatrist Falret described „transitional states” that emerged during the switch from mania to depression and depression to mania (Sedler 1983). He observed that during transitions from depression into mania, vestiges of depression mingled with components of mania; during transitions of mania into depression, vestiges of mania mingle with components of depression.

The first scholarly systematic descriptions of mixed states by Kraepelin and Weygandt date back to 1899 (Weygandt 1899; Kraepelin 1899), and since then their operationalisation, and even their acceptance, have been a matter of controversy (Ghaemi 2008). Kraepelin described mood disorders as a continuum, with „pure“ depression and „pure“ mania being only the extremes of a spectrum that is characterised by three hallmark psychopathological items: Denkstörung (thought disorder), Verstimmung (,ill-humour”, affective disturbance) and Wille (volition). According to Kraepelin each of these three categories can be separately judged as increased or diminished, thus allowing multiple variations of affective states. In his model, only two variations (either all increased or all diminished) constitute the „pure“ poles, the others are mixed states, a concept described more in detail by Wilhelm Weygandt (Weygandt 1899; Salvatore et al. 2002). Kraepelin enumerated six putative subtypes of mixed states depending on the fundamental symptoms of mania or depression. The first three subtypes („depressive-anxious mania“, „excited or agitated depression“, and „mania with thought poverty“) were characterized by an admixture of the three core symptoms of mania (namely, flight of ideas, euphoria, and hyperactivity) and depressive symptoms such as depressed mood, inhibition of thought, and poverty of thought. The next three subtypes („manic stupor“, „depression with flight of ideas“, and „inhibited mania“) were based on the core symptomatology of depression - inhibited thought, depressed mood, and avolition accompanied by manic symptomatology such as euphoria and flight of ideas (Marneros 2001b). To further illustrate Kraepelin's holistic approach towards affective disorders, a diminished speed of thinking or negative thoughts, low mood but increased agitation would characterize what he defined as „depressive Erregung“ (depressive excitement), an affective 
state that today sometimes is labelled ,agitated depression“, although it essentially may rather resemble a bipolar mixed state (Benazzi et al. 2004; Koukopoulos et al. 2007). Of note, the distinct subtypes of mixed states as described by Kraepelin may still hold true and have therapeutic implications as shown by Perugi et al. in his cluster analysis of clinical subtypes of severe bipolar mixed states (Perugi et al. 2013). Also the existence of distinct subtypes of mixed depression- an „excited depression“ subtype (defined by the core feature of psychomotor agitation, and further characterised by talkativeness, irritable mood and distractibility) and a „depression with flight of ideas“ subtype (defined by the core feature of racing/crowded thoughts, and further characterised by risky pleasurable impulses including, among others, those with intense sexual arousal)- was later verified by the work of Akiskal and Benazzi (Akiskal and Benazzi 2004; Benazzi et al. 2004).

Similarly, Caroll proposed a „Neo-Kraepelinian” model of bipolar mixed episodes where the three key dimensions are hedonic function, central pain regulation and psychomotor regulation (Carroll 1983). To explain mixed episodes, Akiskal introduced additionally the concept of temperament. He proposed a model explaining mixed episodes as states arising when an affective episode and temperament are of opposite polarities (e.g., a manic phase in a subject with a depressive temperament or a melancholic phase in a subject with a hyperthymic temperament) (Akiskal 1992). In line with Akiskal's theory, Brieger et al found some evidence that manic mixed episodes occur more often in patients with an inverse, depressive and anxious temperament when examining the temperament of bipolar patients with and without a history of mixed episodes with the TEMPS-a scale (Brieger et al. 2003). However, if we consider temperament as a stable and lasting personal quality, it would be difficult to explain how such a patient can still experience pure manic or depressive episodes at times.

As diverging as the models to explain mixed episodes are the categorical definitions what constitutes a mixed episode. A major problem for including mixed states in studies is the lack of a generally excepted definition (Cassidy et al. 2008; Vieta and Morralla 2010). The DSM IV only recognized mania combined with depression as „mixed states“. No recognition has been accorded to full-blown or major depressive episodes, which may also have excitatory or hypomanic symptoms. According to the DSM-IV (American Psychiatric Association 1994), mixed states imply that diagnostic criteria for a manic episode and a depressive episode (except for the duration criterion) are fulfilled concurrently, following the restrictive concept as originally proposed by Winokur (Winokur et al. 1969). Clearly, these criteria are too 
narrow to be clinically meaningful. It has been shown in post hoc analysis of the pivotal divalproex mania study (Bowden et al. 1994) that already one depressive syndrome predicts inferior responsiveness to lithium and better response to valproate (Swann et al. 1997); a finding, however, that has not been replicated so far.

Indeed, DSM-IV created the most restrictive definition of a mixed episode, whereas DSMIII-R (American Psychiatric Association 1987) still allowed subthreshold manic phases to be included. Clinicians using the ICD-10 (World Health Organization 1992) should be aware that the concept of mixed states in the ICD-10 is more loosely and broadly defined than in the DSM-IV since the exact number of manic or depressive symptoms is not specified (see Table 1). Among those involved in the conception of DSM-IV there was a mutual consent that 2 symptoms of the opposite pole are sufficient for the diagnosis of a mixed state (A. Bertelsen, personal communication). A mixed episode can be diagnosed as a stand-alone episode (F38.00) or as part of bipolar disorder after a previous hypomanic, manic, depressed or mixed episode (F31.6). Different from the definitions of manic and depressed episodes, the ICD-10 does not specify the severity of mixed episodes including whether psychotic symptoms are present or not. Thus, a more detailed description of symptoms and severity of bipolar mixed states in ICD-11 is demanded (Ostergaard et al. 2012).

The concept of dysphoric mania as suggested by Clothier et al. (Clothier et al. 1992) is not well-defined, but sometimes used in the context of drug trials, referring to mania with some depressed features which are either not pronounced enough or too short to fulfil the criteria for a major depressive episode. A more precise, previously used definition are the so called „Cincinnati-criteria” which ask for at least three relevant depressive syndromes that do not overlap with DSM-III-R manic symptoms (McElroy et al. 1992) during a manic episode. This definition came already close to the DSM-5 (American Psychiatric Association 2013) concept of mania with mixed features. Future revisions of the DSM-5 might broaden the current definition of mixed states (Vieta 2016) which is already more inclusive than in the past. Hence, corresponding more to clinical reality DSM-5 abandoned mixed states as a narrowly defined category of its own, and instead re-introduced a broadening of the concept, the mixed specifier; in other words, depression combined with some manic features (,mixed depression”) or mania or hypomania combined with some depressive features („mixed (hypo)mania"). Of note, a manic feature specifier can also be attributed to unipolar depressive disorder. The meaningfulness of this, however, remains controversial, since it can be argued that depressive episodes with mixed features are part of the bipolar spectrum 
(Weibel and Bertschy 2016) as further discussed here below. It has also been noted that a mixed manic feature specifier may easily be attributed to patients with depression and comorbid borderline personality disorder (Perugi et al. 2015). Clearly, moving from DSM-IV to DSM-5 does increase the frequency of mixed episodes: A retrospective chart review of Korean patients diagnosed with BD-I, BD-II or bipolar not otherwise specified according to DSM-IVTR criteria showed that reclassification of patients according to DSM-5 criteria tripled the prevalence of bipolar with mixed features (from 6.0 to $19.6 \%$ ) (Shim et al. 2015).

The diagnosis of a mixed specifier demands the exclusion of affective symptoms due to organic conditions, substance use or medication. However, when affective symptoms outlast the acute physiological effect of substances or medication, a mixed affective specifier can be attributed, similar to DSM-5 diagnostic criteria for a manic or depressive episode. The mixed specifier carries a dimensional perspective on the concept of mixed states, making it more clinically valid, but also causes new problems: for example, anxiety, irritability and agitation, the most common and pronounced symptoms of mixed states, are not included in the concept because of lack of specificity (Vieta and Valenti 2013). Moreover, since the mixed (hypo)manic specifier can also be used in unipolar depression, the validity of a unipolar depression diagnosis in that context can be questioned. The STEP-BD study screened patients initially diagnosed with major depressive disorder for the presence of manic symptoms. Each additional manic symptom led to a $24 \%$ increased chance of a diagnosis of bipolar disorder. Thus, mixed symptoms may indicate bipolarity (Goldberg et al. 2009).

The changing landscape away from categorical definitions towards a dimensional view of psychiatric illness, and especially bipolar mixed episodes, has also been recognized by regulatory agencies. In 2016, the European Medicines Agency (EMA) released a „Concept paper on the need for revision of the note of guidance on clinical investigation on medical products for the treatment and prevention of bipolar disorder”, stating: „Despite the fact that DSM-5 adheres to the categorical classification of psychiatric disorders, dimensional approaches are not uncommon, and often reflect current clinical practice. Bipolar disorder shares symptoms with both Schizophrenia and Major Depression. Subsequently, scientific and regulatory discussion is needed when a population with specific psychopathological characteristics is targeted (e.g., mixed features); where can be extrapolated and where complementary data are needed distinct for the different disorders.” 
In summary, we do not only deal with a wide range of definitions of mixed episodes that have been applied in clinical studies over the recent decades, ranging from as little as one depressive symptom during mania to the narrow DSM-IV definition. We can also expect that regulatory guidance to obtain a label for mixed episodes is under scrutiny and may vary in different countries. As this guideline is meant for use in clinical practice, the task force decided not to restrict evidence to the narrow DSM-IV definition currently used by the FDA for a „mixed episode” label, but consider evidence from studies with wider definitions as equally valuable.

We distinguish between the following treatment situations:

- Acute treatment of a manic episode with $\geq 3$ depressive symptoms as defined by DSM-5 but also including anxiety, irritability and agitation. Agitation alone and its management is covered in a different report (Garriga et al. 2016).

- Acute treatment of a bipolar depressive episode with $\geq 3$ hypomanic or manic symptoms as defined by DSM-5

- Continuation and prophylactic treatment after a mixed index episode. This includes the prevention of relapse or recurrence not only of new mixed, but also of manic or depressive episodes. Whenever possible, we will distinguish between a mixed (hypo)manic and mixed depressive index episode.

- Prevention of mixed episodes after a manic or depressive index episode.

- Evidence derived from studies including mixed patients below the above threshold will also be detailed and valued within the category „Further evidence” but will not be counted for the formal Category of Evidence (CE) system as established in the previous WFSBP Bipolar guidelines.

The bulk of evidence for the WFSBP bipolar task force recommendations for the best available treatment of mixed states will be still based on the DSM-IV definition, and therefore some degree of uncertainty will remain whether these recommendations can be readily extrapolated to other definitions including DSM-5.

\section{Epidemiology and clinical relevance of mixed states}

Not surprisingly, the reported incidence of mixed manic episodes in population-based studies strongly varies depending on study population and definition used, and ranges from less than 
20\% to almost $70 \%$. Using DSM-III R criteria, Cassidy et al (Cassidy et al. 1998) reported on 43 mixed episode patients out of 316 consecutively admitted manic inpatients (14\%). On the other end of the range, Prien et al (Prien et al. 1988) in their NIMH collaborative study found in $67 \%$ of admitted manic patients fulfilment of mixed episode criteria as defined by the authors. The mixed episode criterion was significantly broader: a HAM-D 17 item score of $\geq 7$ while fulfilling RDC criteria (Spitzer et al. 1975) for a manic episode.

If we apply DSM-5 criteria for a manic episode with mixed depressive features, a fair estimate of the incidence might be approximately one third of all manic episodes (Vieta et al. 2014; Young and Eberhard 2015; Reinares et al. 2015). In first episode bipolar disorder, more than $2 / 3$ of episodes show a mixture of manic and depressive symptoms which, however, may not always fulfil threshold criteria for a mixed specifier (Vieta et al. 2014). This is in line with reports that adolescent mania is more likely than adult mania to be characterised by high rates of depressive features or to be mixed (Craney and Geller 2003; Geller et al. 2004; Birmaher et al. 2006). Summarising the different studies looking into gender preference, mixed manic episodes across definitions (mixed mania, dysphoric mania or the manic depressive dimension) are more common in manic women than in men, with the ratio being about 60 : 40 (Gonzalez-Pinto et al. 2007). Other studies report on even higher gender ratios to the disadvantage of women, e.g., (Baldessarini et al. 2014).

In their review of early studies- between 1971 and 1990- in mixed states, Cassidy et al identified the following signs and symptoms possibly relevant to mixed manic states based on selected studies: depressed mood, anxiety, lability, paranoia, hostility, and psychosis (Cassidy et al. 1998). More recently, Malhi et al. pointed out that distractibility and psychomotor agitation may represent the very core of mixed depressive states, as they are more common in patients with mixed depression and bipolar spectrum disorder than in patients diagnosed with unipolar depression or BD-I disorder. Of note, neither distractibility nor psychomotor agitation define a major depressive episode with mixed features (Malhi et al. 2014).

Several distinctive features and predictors of mixed episodes have been described in the literature: Greater female prevalence (Dell'Osso et al. 1991; Akiskal et al. 1998; Arnold et al. 2000)- especially in mixed hypomanic patients (Suppes et al. 2005), more past mixed episodes, higher probability of mixed episodes at illness onset (Perugi et al. 2001), more irritability (Swann 2000) and suicidality (Dilsaver et al. 1994; Strakowski et al. 1996) and neuropsychiatric comorbidity (Himmelhoch and Garfinkel 1986). Manic episodes with or 
without subsyndromal depression also differ in treatment response (Swann et al. 1997; Swann et al. 2002), severity of anxiety (Swann et al. 1986; Swann et al. 2002), and duration (Pacchiarotti et al. 2011).

The depressive burden during a manic episode obviously matters. Young and Eberhard found in a naturalistic study that during their current manic episode, BD-I patients „,with mixed features" according to DSM-5 had more severe symptoms of anxiety, irritability, and agitation (average composite severity score of 4.1 vs 3.4), a higher incidence of suicide attempts (38\% vs $9 \%$ ), and more physician dissatisfaction with treatment response (22\% vs 14\%), compared to patients with $0-2$ depressive symptoms (all $p<0.05$ )(Young and Eberhard 2015). Similarly, when analysing the placebo arm of a RCT testing asenapine in manic and mixed patients, McIntyre at al. showed that with increasing baseline severity of depressive features, treatment outcome became poorer (McIntyre et al. 2013). In line with this, a selfreported online survey (IMPACT) in 700 bipolar patients found that patients with more than three depressive symptoms were more likely to have had a delay in diagnosis, more likely to have experienced shorter symptom-free periods, and were characterized by a marked lower prevalence of typical manic manifestations. The IMPACT survey also assessed non-mood symptoms beyond DSM-5 specifier criteria, including anxiety, agitation, and irritability. Again, anxiety associated with irritability/agitation was a key symptom among patients with DSM-5 mixed features differentiating them from pure manic patients (Vieta et al. 2014).

Most data on the course of illness relate exclusively to BD-I patients; less is known about BD-II mixed states. Tundo et al (Tundo et al. 2015) followed 168 first episode BD-II patients, 12 of them with broadly defined mixed state at illness onset. Mixed state in BD-II was defined according to Koukopoulos criteria of agitated depression (Koukopoulos and Koukopoulos 1999) as prior to DSM-5 BD-II mixed states were „non-existent” in classification systems). Compared to a cohort of 239 Bipolar patients, 52 of them having a mixed episode at onset, having had a broadly defined mixed episode increased the risk of suicidal attempts and substance abuse among patients with BD-II, but not with BD-I.

It remains a matter of controversy whether mixed patients in general are more prone to substance abuse. Some reports suggested a higher rate of comorbid substance abuse in mixed patients (Tohen et al. 1998; Tundo et al. 2015), however, this could not be verified in other studies (Cassidy et al. 2001b). 
Patients with mixed mania have higher rates of recurrence (Hantouche et al. 2006; Tundo et al. 2015), psychiatric re-admissions (Perugi et al. 2000) and a longer duration of illness after the first hospitalisation (Cassidy and Carroll 2001). For planning maintenance treatment in patients with a mixed index episode it is also of note that they are also on a higher risk of switching, probably rapid cycling and suicide attempts which may then call for different therapeutic choices (Azorin et al. 2009). Mixed episodes tend to occur relatively consistently within individual patients as demonstrated by two studies. In a study of hospitalized manic and mixed-manic patients followed prospectively over three episodes, $69 \%$ of mixed-manic episodes were stable from episode 1 to 2 , as were $84 \%$ of manic episodes. The rate of occurrence of mixed-manic episodes in the second episode among patients whose first episode had been mixed was over three times that predicted by chance, indicating much higher persistence of mixed mania across episodes than would occur randomly (Cassidy et al. 2001a). In a retrospective study of 253 bipolar patients hospitalized at least twice because of consecutive manic or mixed episodes over 20 years, depressive mood, irritability, psychomotor inhibition, psychosis, and mania were highly stable over both consecutive recurrences, and over the long-term course of illness. This syndromal stability was not substantially affected by gender, age of onset, or intermittent substance abuse (Sato et al. 2003). A recent meta-analysis of large naturalistic studies confirmed that mixed states begets mixed states: A mixed index episode was in almost half of the cases followed by another mixed episodes (45.9\%, vs. 28\% depressive and 26.1\% manic (Radua et al. 2017).

Little is known about Quality of Life (QoL) in patients with mixed states opposed to pure affective states. As the result of a small interview series, Mortensen et al. noted that participants described mixed states as worse than other bipolar disorder states but their residual symptoms as prolonged. Mixed states affected the functioning of patients in key life domains such as self-esteem, family, love and social life, physical well-being, and working capability (Mortensen et al. 2015).

\section{Continuation and maintenance treatment after an acute mixed episode}

Long-term treatment in bipolar patients has been traditionally divided into continuation and maintenance (or prophylactic) treatment, which are, in turn, associated with the starting points „remission” and „recovery”, respectively. In the WFSBP guideline on long term treatment (Grunze et al. 2013) we extensively elaborated on the definitions and timelines of continuation and prophylactic treatment, and its implications for study designs. Due to the paucity of distinct data for continuation and prophylactic treatment after an acute mixed 
episode, we will not elaborate on this in this guideline and separate continuation from prophylactic treatment, but use a simplified approach by combining them into one category „Maintenance”, simply implying the aim of preventing the emergence of symptoms following remission of an episode.

\section{Prevention of mixed episodes after a manic or depressive index episode}

In patients with a history of mixed episodes, prophylactic treatment after a manic or depressive episode should not only target the prevention of manic or depressive relapse, but also give special attention to mixed recurrences. This is particularly true if a prophylactic treatment has been chosen that might increase the risk of provoking a mixed state, e.g., long term antidepressant continuation after a depressive index episode (El-Mallakh et al. 2008), or antipsychotic- induced dysphoria after a manic index episode (Wu and Okusaga 2015). Unfortunately, only few maintenance studies supply specific information on the prevention of mixed episodes. Especially with older studies, this information is lacking.

\section{Scope of this review}

Parts I, II and III of the World Federation of Societies of Biological Psychiatry (WFSBP) guidelines for the biological treatment of bipolar disorders (Grunze et al. 2009; Grunze et al. 2010; Grunze et al. 2013) concerned the acute and maintenance treatment of mania and bipolar depression. At the time of compilation of the acute treatment guidelines, there was only little data available on the evidence-based treatment of mixed episodes, especially mixed depressive episodes and prophylaxis of mixed episodes. In the acute mania guideline (Grunze et al. 2009), the efficacy of medication also in mixed manic episodes was detailed and included in the recommendations if such information was available, mostly from secondary post hoc analysis of RCTs.

Due to the existing evidence, this guideline has its primary focus on mixed episodes in BD-I disorder. When evidence is available, we will also consider results from studies in BD-II disorder and rapid cycling patients and include them into our ranking of evidence. As the evidence has been derived by large from studies in adults aged 18-65, this guideline is primarily only applicable to this patient group. This issue is particularly relevant for this guideline because mixed states and rapid cycling are more frequent in younger patients than adults (Grande et al. 2016). In the few cases where additional information for efficacy or safety in children or old age was retrieved, we also cited it in the body of text but did not 
include it for primary efficacy ratings, but as additional supportive/non-supportive evidence (category „Further evidence (FE)”).

This guideline series is dedicated to Bipolar Disorder as categorized in DSM-IV and DSM-5. Although it is feasible to assume that a unipolar MDE with mixed features according to DSM-5 may truly fall into the bipolar spectrum, its treatment is not part of this guideline. Instead, we would like to refer the reader to a recent expert guidance on depressive mixed states (Stahl et al. 2017). It has been hypothised that this new diagnostic designation is simply a precursor of bipolar disorder and that patients meeting these criteria should be treated as such (Benazzi 2005; Benazzi 2006). At this time, controlled evidence for treatment is virtually restricted to lurasidone (Targum et al. 2016; Suppes et al. 2016b), and in the absence of further evidence, this expert consensus suggests to use similar principles of treatment in bipolar and unipolar depression with mixed features- avoidance of antidepressant monotherapy and preferred use of some atypical antipsychotics or mood stabilizers. Whether this approach will result in better outcomes is still up for further investigation.

As with the previous guidelines we did not include schizoaffective disorders per se despite their wide similarities with bipolar disorder (Marneros 2001a) as it was felt that such a broad spectrum view would go beyond the scope of this paper. However, in patients with mixed episodes psychotic symptoms are not rare (Gonzalez-Pinto et al. 2011), and in clinical practice, a clear delineation of a psychotic mixed bipolar episode from a schizoaffective disorder with mixed features appears often impossible and without therapeutic consequences (Murru et al. 2016).

Besides efficacy, we will also consider safety and tolerability issues. Unfortunately, these important issues are not uniformly captured across studies and seldom measured as rigorously as efficacy; thus, any in-depth grading of these important aspects is difficult and subject to bias.

As with the previous guidelines of this series, we concentrate on pharmacotherapy and physical therapies. We value the contribution of psychotherapies and sociotherapies as part of a modern and individual treatment package; however, a full evidence based review of these modalities is beyond the scope of the present paper.

For each medication, we will supply a grading of efficacy in monotherapy and in combination/augmentation treatment. At the end, this guideline aims to supply the reader with the following information for a specific medication (when available): 
- Efficacy in the treatment of acute manic mixed episodes

- Efficacy in the treatment of acute depressive mixed episodes

- Efficacy in maintenance treatment after an acute mixed episode in preventing episodes of any polarity or a manic, depressive or mixed episode if such detailed information is available

- Efficacy in maintenance treatment after an acute manic or depressed episode in preventing new mixed episodes

- Further important supportive/unsupportive evidence, e.g. from large scale naturalistic studies, post -hoc analyses of very small numbers from RCTs, or in specific subgroups, e.g. children, adolescents, old age („,Further evidence (FE)”)

- Brief summary of the acute and long-term safety and tolerability of the medication (,Safety \&Tolerability (ST)”) as this has already extensively dealt with in the previous guidelines within this series (Grunze et al. 2009; Grunze et al. 2010; Grunze et al. 2013).

- Grades of recommendation for the respective treatment scenarios ((„,Recommendation grade $(R G)$ ')

For practicability of use and antisuicidal properties of a medication, we refer the reader to the 2013 bipolar maintenance guideline.

\section{Methods of this review}

The methods of retrieving and reviewing the evidence base, and coming up with a recommendation are by large identical to those described in the WFSBP guideline for acute mania and bipolar depression (Grunze et al. 2009; Grunze et al. 2010). For those readers who are not familiar with these guidelines, we will summarize the methods in brief.

The data used for these guidelines have been extracted from a MEDLINE and EMBASE search, the Science Citation Index at Web of Science (ISI) and a check of the Cochrane library for recent meta-analyses and from recent proceedings of key conferences. The original search was conducted on May 29, 2013, and had a final update on March 12, 2017. Some reviews mentioned in this article have been fully published after this date but were available to the authors as accepted manuscripts. Results of the initial search have also been reviewed previously (Grunze and Azorin 2014). To ensure comprehensiveness of data, we also 
consulted various national and international treatment guidelines, review papers, consensus statements, and hand-searched several textbooks. In addition, www.clinicaltrials.gov was accessed to check for unpublished studies, and if possible, the sponsors of those trials were approached for further information. All searches cover the time span from 1967 to March 2017. Having different sources of information appears crucial: A systematic MEDLINE search for a recent meta-analysis of the prevalence of generalized anxiety disorder (GAD) in BD returned 1300 results but identified only 7 out of 30 eligible articles whereas the rest was identified by hand search in reviews, text books etc. (Preti et al. 2016).

To be eligible, studies had to enrol patients with mixed mania/ mixed depression, or with both mixed and pure episodes. We retrieved evidence from long and short-term randomized controlled medication trials (RCTs), open-label studies, case series or reports, and retrospective and prospective studies. In the latter case, data were from stratified, post hoc, or treatment interaction analyses and reporting results on the mixed subgroup. We retrieved data on other physical therapies, such as electroconvulsive therapy (ECT) or deep brain stimulation (DBS). We excluded publications in languages other than English, French, German, or Spanish, containing duplicated data, and with no data stratified by mixed and pure manic or pure depressive patients.

Given the large heterogeneity of study designs and diverging definitions of mixed states, we did not use the results of meta-analyses as evidence of the same level as results from single RCTs fulfilling inclusion criteria. Meta-analysis cannot resolve uncertainty where the methodology of the individual RCTs is flawed (Goodwin et al. 2016). Those meta analyses that we consulted if the evidence remained unclear based on single studies were selected according to the quality criteria as defined by Huf et al (Huf et al. 2011). An exception is the category „safety” in case we deal with rare events that may only light up when large numbers of subjects are analysed across trials.

Network meta-analyses as recently conducted for acute mania (Yildiz et al. 2015), bipolar depression (Taylor et al. 2014) and bipolar maintenance (Miura et al. 2014) appear methodologically more sound and may allow a more objective ranking of drugs according to their efficacy and acceptance. However, to our knowledge no such network analysis has been published to date covering all potential, evidence based treatments for mixed states; only for the acute treatment with atypical antipsychotics have two meta-analyses been conducted: one 
for mixed mania (Muralidharan et al. 2013) and one for mixed depression (Fornaro et al. 2016).

In this review, evidence derived from combination treatments will contribute equally to the final recommendation grade as evidence derived from monotherapy with the respective drug, and we will discuss the respective combination/augmentation studies under the respective monotherapy header, considering the constant drug + placebo as the „Placebo-condition”, and the constant drug + the investigational drug as the „test-condition”. Clearly, some uncertainty of this approach remains, as we cannot exclude that the test drug is only beneficial in the specific combination, but not by itself.

In order to achieve a uniform and, in the opinion of this taskforce, an appropriate ranking of evidence we adopted a similar rigorous hierarchy of evidence and level of recommendation as was used in previous WFSBP guidelines including the first published WFSBP Guidelines for the Pharmacological Treatment of Anxiety, Obsessive-Compulsive and Post-Traumatic Stress Disorders and its updates (Bandelow et al. 2008a) (see Table 2). In brief, a drug must have shown its efficacy in double-blind placebo-controlled studies in order to be recommended with substantial confidence (Categories of evidence (CE) A or B, corresponding to RGs 1-3). Lower level evidence from uncontrolled studies (CE „C”) or conflicting results (CE „D”) were accepted for a low RG 4 or 5, respectively. Substantial concerns about safety and tolerability of a drug could also result in a downgrading of the RG, especially when making a distinction between RG 1 and 2 .

Depending on the number of positive trials and the absence or presence of negative evidence, different CE for efficacy were assigned. A distinction was also made between „lack of evidence” (i.e., studies proving efficacy or non-efficacy do not exist, CE „F”) and „negative evidence" (i.e. the majority of controlled studies shows non-superiority to placebo or inferiority to a comparator drug (CE „E”). When there is lack of evidence, a drug could still reasonably be tried in a patient unresponsive to standard treatment, while such an attempt should not be undertaken with a drug that showed negative evidence.

We set a minimum of 25 participants for a placebo- controlled study to be considered as evidence for the categories of evidence A or B. RCTs with a smaller number of participants could still be considered for the categories „C” or „Further evidence (FE)” depending on their overall quality. 
The role and positioning of post hoc and subgroup analyses has not been clearly defined in the first guidelines of the WFSBP series using our grading system (Bandelow et al. 2008b). Post hoc secondary analyses play a prominent role in studies including manic and mixed manic patients but bear the risk of inflating the chance of false-positive findings (Oxman and Guyatt 1992). Many of these analyses were done on data sets that have been not informative in their primary outcome, were not hypothesis generated, and therefore will be counted as CE "C" (similar to uncontrolled studies). However, when a secondary analysis has been included a priori in the analysis plan and is sufficiently powered, a CE „, B” could be considered.

Another deviation from the original WFSBP guideline grading system is the role of large-size registry studies. Of course, they do have the risk of bias, similar but of a different kind than with RCTs. On the other hand, they can supply valuable information about the real world effectiveness and acceptance of treatment modalities, and especially in under-researched or rare indications (as mixed states are to some degree) they can help to resolve uncertainity. The task force decided to accept registry studies at least on $\mathrm{CE}$ "C $\mathrm{C}$ " level (as other retrospective studies), but if they are of good quality and bias is minimized, e.g. each patient serving as his own control in an on-off design, they can receive the same CE "B" as a RCT. This decision of the task force is also in line with their appreciation in other recent bipolar guidelines, e.g. the BAP guideline (Goodwin et al. 2016).

Further evidence (FE), and safety and tolerability (ST) were graded with a simplified system ranging from „,++” for best positive evidence to „ - -“ for strong negative evidence and rated separately for acute and long-term treatment (see Table 3).

Recommendations were derived from the CE for efficacy and from additional aspects as safety and tolerability. We have not considered the direct or indirect costs of treatments as these vary substantially across different health care systems. Additionally, as the approval by national regulatory authorities is dependent on a variety of factors, including the existence of a label „mixed episodes” and the sponsor's commercial interest (or lack thereof) this guideline is exclusively based on the available evidence.

The task force is aware of several inherent limitations of these guidelines- namely publication bias (Turner et al. 2008; Flint et al. 2015), sponsor bias (Lundh et al. 2012), and, finally, the limitations of evidence itself. One of the most important clinical questions that cannot be sufficiently answered in an evidence based way is what to do when any first step treatment fails, which is in mixed states rather is the rule rather than the exception. Therefore, with the 
current level of knowledge we can only provide suggestive guidelines and not rigorous algorithms.

Once a draft of this guideline had been prepared by the Secretary and co-authors, it was sent out to the 63 members of the WFSBP Task Force on Treatment Guidelines for Bipolar Disorders for critical review and addition of remarks about specific treatment peculiarities in their respective countries. A second draft, revised according to the respective recommendations, was then distributed for final approval.

These guidelines were established without any financial support from pharmaceutical companies. Experts of the task force were selected according to their expertise and with the aim to cover a multitude of different cultures.

\section{Medications commonly used in mixed states and their ranking by evidence}

The initial search of the literature led to the identification of 1,388 publications for further evaluation. After the exclusion of non-relevant articles for this guideline we extracted data on the efficacy outcomes of medications or physical therapies for acute or maintenance treatment of mixed episodes from 133 studies. Briefly, most of the studies reported results for adult populations (89.5\%), were performed in inpatients (55\%), were based on short-term treatments (70\%), and reported data obtained from RCTs (37.6\%; followed by open-label studies [24\%], case reports [17.3\%], retrospective evaluations [15\%], and prospective observations [6\%]). The vast majority of data was therefore from uncontrolled studies (62.4\%), and was considered as lower grade evidence of efficacy of the related therapy ${ }^{1}$. The update of the search on March 16, 2017, identified another 31 additional studies contributing to the evidence for a given treatment.

In the following chapters, we will highlight pivotal studies supporting (or speaking against) efficacy of a given medication, amended by other supportive evidence if clinically relevant. We assigned ratings for efficacy as detailed in the chapter on „Methods of this review”, and

\footnotetext{
${ }^{1}$ The appropriate grading of open, but randomized large studies and of large, prospectively collected data sets with a quasi experimental designs with patient-relevant outcomes in comparison to small RCTs triggered a vivid discussion among the task force members. Following the logic of the recent BAP Guideline (Goodwin et al. 2016) ,the recent commitment of the NIH to prioritize effectiveness studies (Lauer and Collins 2010) and based on the quantity and quality of the data, and reliability of the source, it was finally decided to attribute the study of Joas et al (2017) the same weight as a RCT.
} 
graded the categories „Further evidence (FE) and „Safety and Tolerability” in a more simplified system (ranging from ++ to - -, see Table 3). As this guideline should be useful for the practicing clinician, drugs under consideration are not exclusively those where data of randomized controlled studies are available, but those which are either used with some trust and frequency by clinicians in bipolar patients, e.g. antidepressants as a group, or in specific subgroups, e.g. clozapine in otherwise treatment refractory patients.

\section{Antidepressants}

Almost all guidelines recommend not initiating antidepressants when a mixed state is diagnosed, although in clinical practice they remain widely prescribed together with antimanic agents for the treatment of mixed states (Azorin et al. 2009). The International Society for Bipolar Disorders Task Force on antidepressants provided guidance in that regard (Pacchiarotti et al. 2013). Mixed states are best conceptualised, for both diagnostic assessment and treatment purposes, as states of tremendous mood dysregulation and instability rather than just states characterized by either the simultaneous or sequential presence of various combinations of depressive and manic/hypomanic symptoms. This does not only constitute a major shift in paradigm in terms of the conceptualisation; it also has implications for the treatment of mixed states. If mixed states were to be perceived as a combination of depressive and manic symptomatology, then the use of a combination of mood stabilizers or antipsychotics and antidepressants would appear to be an intuitive option. If the focus of treatment is on the regulation of mood and the correction of mood instability, however, then it becomes apparent that medication with potentially mood-destabilising effects, e.g., some antidepressants and typical antipsychotics, should be avoided (Wehr and Goodwin 1987; Akiskal 1994). Current evidence from prospective or retrospective studies support the appreciation that mixed patients are at higher risk for a maniform switch (Bottlender et al. 2002; Strejilevich et al. 2011; Valenti et al. 2011). The use of antidepressants in bipolar disorder has also been associated with the de-novo-induction of mixed states (Dilsaver and Swann 1995; El-Mallakh et al. 2008). However, it should be borne in mind that the data mentioned here above comes from observational studies and case reports. Whether antidepressants can provoke mixed episodes during maintenance treatment in remitted patients is unknown. These considerations imply that mixed states should be treated, if unavoidable as a last resort, only with selected antidepressants known to be less likely to induce a treatment emergent affective switch (TEAS), and with minimal duration and dosage. 
Based on the circadian rhythm hypotheses of mixed features where mixed features express a state of transitional unstable circadian rhythm (Lee et al. 2013), the use of antidepressants such as agomelatine may be advantageous; however, no clinical data on its use in mixed states have been published, and a pivotal RCT in bipolar depression with adjunctive agomelatine failed (Yatham et al. 2016).

For adjunctive treatment post hoc data are available for fluoxetine added to olanzapine (Tohen et al. 2003b) in depressive mixed states. This 8-week, double-blind trial of adult BD-I depression compared treatment with placebo, olanzapine or olanzapine/fluoxetine combination (OFC). Studying mixed depression was not a priori goal of the double-blind trial, but among the study participants (diagnosed with BD-I disorder, current episode MDE according to DSM-IV) a fair number of patients having $\geq 2$ manic/hypomanic symptoms (i.e., $\geq 2$ Young Mania Rating Scale [YMRS] items scoring $\geq 2$ ) were identified. Response was defined as a $\geq 50 \%$ reduction in Montgomery-Asberg Depression Rating Scale score and $<2$ concurrent manic/hypomanic symptoms. Of the patients with mixed depression (which were almost 50\% in all arms), patients treated with OFC showed a significantly higher response rate versus patients treated with placebo (OR = 3.91; 95\% CI, 1.80-8.49; $\mathrm{p}=.0006$ ), but no statistically significant difference was observed between OFC and olanzapine in the rate of responders $(\mathrm{OR}=2.00 ; 95 \% \mathrm{CI}, 0.96-4.19 ; \mathrm{p}=.065)$. Thus, in summary, addition of fluoxetine to olanzapine did lead to a numerical but not a statistically significant increase of responders in mixed depression. No worsening of mania was seen among the fluoxetine treated patients (Benazzi et al. 2009). Categorisation of the evidence from this study in our grading system is difficult; as it was not an a priori planned post hoc analysis, it would count as „C” evidence if positive. Assigning a category „E”, however, for negative evidence may be not justified given that it is a single exploratory analysis, whereas the definition of category „E” asks for a „majority of studies” that are negative. Thus, category „F” (lack of evidence) may be most appropriate.

CE in acute manic mixed episodes (monotherapy) is „F”.

CE in acute manic mixed episodes (combination/ augmentation therapy) is „F”.

CE in acute depressive mixed episodes (monotherapy) is „F”.

CE in acute depressive mixed episodes (combination/augmentation therapy) is „F”

CE to prevent a new episode after a mixed index episode (monotherapy) is „F”. 
CE to prevent a new episode after a mixed index episode (combination/augmentation therapy) is „F”.

CE to prevent a mixed episode after a manic or depressed index episode (monotherapy) is „F”.

CE to prevent a mixed episode after a manic or depressed index episode (combination/augmentation therapy) is „F”.

Rating of FE: 0 for short-term treatment, 0 for long- term treatment

Rating of ST: - for short-term treatment, 0 for long- term treatment

\section{Recommendation grade (RG)}

None. Based on the evidence, the use of antidepressants for the acute and continuation treatment of mixed episodes or for the prevention of mixed episodes cannot be recommended and may be potentially hazardous.

\section{Aripiprazole}

\section{Efficacy in acute manic mixed episodes}

The first pivotal RCT of aripiprazole in acute manic or mixed patients included 86 subjects (33\%) with DSM-IV mixed states (Keck et al. 2003a). Unfortunately, separate results for pure manic and mixed patients have not been reported in the paper. Aripiprazole was then studied in a second 3-week RCT that again included both DSM-IV manic and mixed patients with the subgroup of mixed patients now consisting of 113 subjects. As confirmed by the principal investigator, the subgroup analysis in mixed patients was part of the protocol and planned a priori and not post hoc. In DSM-IV mixed episode patients, aripiprazole produced significantly greater improvements from baseline compared with placebo in YMRS total score $(\mathrm{P}=0.01)$, and significantly greater improvements from baseline in Montgomery-Åsberg Depression Scale [MADRS] total score compared with placebo at endpoint $(\mathrm{P}=0.04)$ (Sachs et al. 2006). The effect of aripiprazole on depressive symptoms in mixed patients appears genuine given the mean baseline severities and improvements of MADRS total scores (mean baseline: aripiprazole 18.39, placebo 20.06, both corresponding to mild to moderate depression when measured in pure depression; mean change: aripiprazole -7.93; placebo 4.29, $\mathrm{P}=0.041$ ) However, in mixed patients depression scores may be inflated by unspecific symptoms also present in mania, e.g. sleep (MADRS item 4) and concentration (MADRS 
item 6) problems. A single item analysis of the MADRS scores in this study were not available to the authors, and we cannot rule out that improvement of depression was largely due to improving symptoms overlapping in mania and depression rating scales

The mean dosage of aripiprazole was $27.2 \mathrm{mg} / \mathrm{d}$, which is in line with recommended dosages for acute mania, but considerably higher than what has been successfully tested as augmentation treatment for unipolar depression. When these patients were pooled with the subgroup of mixed patients of the first similarly designed RCT (Keck et al. 2003a) aripiprazole was found to be superior to placebo in improving manic symptoms in both manic and mixed subgroups and regardless of the severity of depressive symptoms. Moreover, aripiprazole was associated with higher percentages of responders and remitters than placebo regardless of patients presenting with a manic or mixed episode $(\mathrm{p}=0.0006$ for responder rates, and $\mathrm{p}=0.01$ for remission rates in mixed patients)(Suppes et al. 2008).

No separate outcome analyses have been published for further RCTs including manic and DSM-IV mixed patients, namely the positive studies by Young et al (Young et al. 2009),Keck et al (Keck et al. 2009a) and Zimbroff et al (Zimbroff et al. 2007), and the negative acute study by El-Mallakh et al. (El-Mallakh et al. 2010) despite including reasonable numbers of mixed patients. Another acute RCT, the AMAZE study, conducted in an Asian population, included only 28 DSM-IV mixed patients, a sample too small to allow for a separate meaningful analysis (Kanba et al. 2014). A randomized, double-blind comparison of aripiprazole against haloperidol was also underpowered for a separate analysis of mixed patients (Vieta et al. 2005).

We identified one RCT testing aripiprazole in combination/augmentation therapy in acute manic and DSM-IV mixed episodes (Vieta et al. 2008b). Again, no separate analysis for mixed patients has been reported in the paper.

$C E$ in acute manic mixed episodes (monotherapy) is „ $B$ ” both for manic and depressive symptoms.

CE in acute manic mixed episodes (combination/augmentation therapy) is „ $F ”$.

\section{Efficacy in acute depressive mixed episodes}

No data is available for the acute treatment of DSM-5 bipolar depression with mixed features or otherwise classified depressive mixed states.

CE in acute depressive mixed episodes (monotherapy) is „F”. 
$C E$ in acute depressive mixed episodes (combination/augmentation therapy) is „F”.

\section{Efficacy in maintenance treatment after an acute mixed episode in preventing episodes of any polarity or a new manic, depressive, or mixed episode}

For the 48 patients with mixed index episodes participating in the aripiprazole oral continuation studies (Keck et al. 2006; Keck et al. 2007) no separate outcome data have been reported. A recently finished study (ClinicalTrials.gov Identifier: NCT01567527) testing aripiprazole long-term injectable included only manic patients at baseline.

Yatham et al. (Yatham et al. 2013) conducted a post hoc analysis of a 52-week maintenance combination study (aripiprazole + lithium or valproate vs placebo + lithium or valproate, 107 patients with DSM-IV mixed mania at entry but stabilized at baseline (ClinicalTrials.gov Identifier: NCT00261443, original publication (Marcus et al. 2011)). They found no significant advantage of aripiprazole for the group of mixed patients for time to any relapse. Another smaller maintenance study comparing aripiprazole+ valproate vs placebo+ valproate after a manic or mixed index episode included only a very small number of mixed patients, and separate outcomes have not been reported (Woo et al. 2011).

Post hoc interaction analysis of a 52-week study testing lamotrigine + aripiprazole vs lamotrigine + placebo (Carlson et al. 2012) demonstrated that there were differential treatment effects according to a patient's index episode, manic or mixed presentation, for time to depressive episode ( $\mathrm{p}=0.044$ ). The results from subgroup analyses showed that time to relapse to a depressive episode was significantly longer with the aripiprazole combination compared with the placebo combination in the subgroup of the 173 patients presenting with a mixed episode at baseline $(\mathrm{p}=0.041)$ but not in the subgroup of patients with a manic episode at $(\mathrm{p}=0.468)$. For any relapse, manic or mixed relapses, no separate data have been reported in the original paper. As a separate analysis of pure manic and mixed patients is not listed as primary or secondary outcome on clinicaltrials.gov (ClinicalTrials.gov Identifier NCT00277212) we have to assume that this analysis has not been part of the original investigational plan; thus, according to our criteria, is will not be eligible for a CE „B” rating, but only CE „C”.

CE to prevent a new episode after a mixed index episode (monotherapy): is „F”.

CE to prevent a new episode after a mixed index episode (combination/augmentation therapy): „E” for „any” (in combination with lithium or valproate), „F” for „manic” and „mixed”, „C” for depressive (in combination with lamotrigine). 


\section{Efficacy in maintenance treatment after an acute manic or depressed episode in preventing new mixed episodes}

For the 48 patients with manic index episodes participating in the aripiprazole oral continuation studies (Keck et al. 2006; Keck et al. 2007) no separate outcome data for mixed recurrences have been reported. The same is true for the combination studies (Marcus et al. 2011; Carlson et al. 2012).

Some recent data are available for aripiprazole long-term injectable. A randomized, doubleblind, placebo-controlled trial assessed the time to recurrence of any mood episode in BD-I disorder after maintaining stability on aripiprazole long-term injectable for at least 8 weeks. Study entry criterion was a manic index episode with an YMRS total score $\geq 20$. The absolute number of mixed recurrence was small (11 out of 103 recurrences in total). At study end (week 52) the investigators found a reduction in mixed recurrences according to DSMIV-TR criteria with aripiprazole long-term injectable compared to placebo that just missed significance $(p=0.06)$ (Calabrese et al. 2017).

CE to prevent a mixed episode after a manic or depressed index episode (monotherapy) is „E” for a manic index episode and „F” for a depressive index episode.

CE to prevent a mixed episode after a manic or depressed index episode (combination/augmentation therapy) is „F”

\section{Further evidence (FE)}

In a 24-week, observational, prospective study in Taiwanese adolescents with a manic, depressive or mixed episode, aripiprazole was effective in reducing the CGI severity and BPRS total scores from baseline to endpoint. However, aripiprazole performed significantly better in manic than in depressive or mixed patients, and improvement of BPRS ratings in mixed patients was only marginal (Tang et al. 2010).

Rating of FE: + for short-term treatment, 0 for long- term treatment

\section{Safety \&Tolerability (ST)}

In the combined analysis of the two 3- week acute studies the most common TEAS that occurred in more patients receiving aripiprazole were somnolence, dyspepsia, akathisia, and accidental injury (Suppes et al. 2008).

In a 52-week study comparing aripiprazole and lithium and including a significant number of DSM-IV mixed index episode patients (27 out of 63), modest increases in body weight were 
observed in both groups: $+0.97 \mathrm{~kg}(2.1 \mathrm{lb})$ for aripiprazole $(\mathrm{n}=127)$ and $+0.74(1.6 \mathrm{lb})$ for lithium ( $\mathrm{n}=136), \mathrm{P}=.60$. A significant difference in body weight increase was observed only among patients with a BMI < 25: $+2.66 \mathrm{~kg}(5.9 \mathrm{lb})$ for aripiprazole $(\mathrm{n}=35)$ and +0.40 $\mathrm{kg}(0.9 \mathrm{lb})$ for lithium $(\mathrm{n}=37), \mathrm{p}=.02$. Mean changes from baseline to week 52 in fasting levels of total cholesterol, high-density lipoprotein cholesterol, low-density lipoprotein cholesterol, plasma glucose, triglycerides, or insulin (last observation carried forward) were small in both aripiprazole and lithium treatment groups; no significant differences were observed. Mean laboratory values were within the normal or borderline range for both treatment groups across all BMI categories (McIntyre et al. 2011).

aripiprazole is in the US Food and Drug administration (FDA) „C” pregnancy category, meaning that risk cannot be ruled out as human studies are lacking, and animal studies are either positive for foetal risk or lacking as well (Nguyen et al. 2009).

Rating of ST: 0 for short-term treatment, +for long-term treatment

\section{Recommendation grade (RG)}

The $R G$ is „3” for the acute treatment of manic mixed episodes for manic and depressive symptoms

The RG is „4” for prevention of depression after a mixed manic index episode (in combination with lamotrigine).

\section{Asenapine}

When asenapine entered the market, there was already a large portfolio of antimanic treatments available and thus an obvious need to demonstrate additional benefits. Hardly any medication became subjective to so many post hoc analyses of two acute monotherapy studies (McIntyre et al. 2009; McIntyre et al. 2010b). Probably as a result of those post hoc analyses, clinicians tend to use adjunctive asenapine in patients with less severe manic symptoms but more complex clinical profile, including more mixed episodes in the past as shown by a recent survey (Grande et al. 2015). Thus, it is of interest to see whether this clinical usage is fully reflected by the evidence.

\section{Efficacy in acute manic mixed episodes}

The efficacy of asenapine in mixed states was first studied as monotherapy vs. placebo and vs. olanzapine as active comparator in a short-term 3-week trial in 488 patients with DSM-IV 
diagnosis of manic / mixed episodes (McIntyre et al. 2009). A post hoc ANCOVA with LOCF on a modified intend-to -treat (ITT)-population found that the subgroup of patients with a mixed episode at baseline $(n=150)$ had only a trend towards significant mean changes in manic symptoms with asenapine vs. placebo $(\mathrm{p}=0.05)$, whereas olanzapine was superior to placebo $(\mathrm{p}<0.006)$. Using MMRM analysis, both asenapine and olanzapine missed significance in the mixed patient subgroup. A second, identically designed acute study included 158 patients with DSM-IV mixed episodes; however, separate analyses for manic and mixed patients have not been reported for this study. Both acute studies (McIntyre et al. 2009; McIntyre et al. 2010b) also captured changes of depressive symptomatology finding significant superiority vs placebo for olanzapine, but not asenapine. However, they do not supply separate post hoc data for reduction of depressive symptoms in mixed patients.

A combined post hoc analysis of the two acute studies reporting on the whole mixed population of 302 patients analysed DSM-IV mixed patients as a separate group, and in addition subgroups of patients with significant depressive symptoms as defined by the authors (MADRS total score $\geq 20$ or Clinical Global Impression for Bipolar DisorderDepression severity scale (CGI-BP-D) severity score $\geq 4$ ) which are likely to correspond to patients with more extended definitions of mixed episodes (Szegedi et al. 2011). In all three groups, asenapine (but not olanzapine) was significantly superior to placebo in reducing LS mean changes in baseline MADRS total score, with higher effect sizes in patients with significant depressive symptoms.

Finally, a further post hoc analysis of the two pivotal acute studies (McIntyre et al. 2009; McIntyre et al. 2010b) combined two criteria of the analysis of Szegedi et al (Szegedi et al. 2013) and examined a subgroup of patients $(n=98)$ meeting criteria both for DSM-IV-TR criteria for mixed episodes and a baseline MADRS total score $\geq 20$ (Berk et al. 2015). Decreases in MADRS scores (LS mean $\pm S E$ ) were significantly greater in the asenapine group than in the placebo group from baseline to day $21(-14.03 \pm 2.01$ vs $-7.43 \pm 2.09 ; \mathrm{P}=$ .0264), and endpoint (LOCF analysis, $-10.71 \pm 1.76$ vs $-5.19 \pm 1.98$; $\mathrm{P}=.039$ ). Decreases in Young Mania Rating Scale mean total score were greater also with asenapine than with placebo (-16.58 vs $-10.27 ; \mathrm{P}=.0229)$.

We identified one study in acute manic $(n=198)$ or mixed states $(n=126)$ investigating asenapine in combination with lithium or valproate (Szegedi et al. 2012). Asenapine was effective in the overall study population in reducing YMRS score from baseline to endpoint 
(day 21), but not different from placebo in reducing depressive symptoms. No separate analysis for mixed patients has been supplied.

In conclusion, the evidence from post hoc analysis is negative for asenapine monotherapy to be effective against acute manic symptoms (McIntyre et al. 2009), whereas there is evidence from combined post hoc analysis for efficacy against depressive symptoms (Szegedi et al. 2011). For combination treatment, no separate data for mixed patents have been published.

CE in acute manic mixed episodes (monotherapy) is „E” for manic symptoms, and „C” for depressive symptoms ${ }^{2}$.

CE in acute manic mixed episodes (combination/augmentation therapy) is „F”.

\section{Efficacy in acute depressive mixed episodes}

We could not identify a study testing asenapine in depressive mixed episodes or a bipolar MDE with a manic feature specifier ${ }^{3}$. Although tempting, extrapolation from post hoc analyses of mixed mania studies with prominent depressive symptoms (Szegedi et al. 2011; Berk et al. 2015) should not been made as their key diagnostic feature is still full criteria of a manic episode.

CE in acute depressive mixed episodes (monotherapy) is „F”.

CE in acute depressive mixed episodes (combination/augmentation therapy) is „F”.

\section{Efficacy in maintenance treatment after an acute mixed episode in preventing episodes of any polarity or a new manic, depressive, or mixed episode}

Two 40 week extension studies of acute trials comparing asenapine with olanzapine have been conducted; one in monotherapy including patients who had finished the two acute trials (McIntyre et al. 2010a) and one in combination treatment including those patients recruiting from patients who finished the combination acute study (Szegedi et al. 2012). None of them

\footnotetext{
${ }^{2}$ Although the combined post hoc analysis by Szegedi et al, 2013 and Berk et al, 2015, are conducted in reasonably large samples, it is not apparent whether they have been planned a priori as part of the investigational plan and study protocols. Therefore, the grading is „C”, not „B”.

${ }^{3}$ The title of the analysis of Berk et al (2015) „Effects of Asenapine in BD-I Patients Meeting Proxy Criteria for Moderate-to-Severe Mixed Major Depressive Episodes: A post hoc Analysis” is misleading; patients are still defined by having a DSM-IV Mixed Episode which implies the preponderance of mania. This is also reflected by the YMRS baseline severity of $>27$ in all study arms. For this reason, the task force also feels that the inclusion of this post hoc analysis in the meta-analysis by Fornaro et al entitled „Atypical Antipsychotics in the Treatment of Acute Bipolar Depression with Mixed Features: A Systematic Review and Exploratory MetaAnalysis of Placebo-Controlled Clinical Trials" (Fornaro et al. 2016) is not justified.
} 
supplied separate information on outcomes for patients with a mixed index episode. Both studies had their primary focus on safety, not efficacy, and numbers of mixed patients entering and finishing the extension studies are very small.

CE to prevent a new episode after a mixed index episode (monotherapy)is „F”.

CE to prevent a new episode after a mixed index episode (combination/augmentation therapy) is „F”.

\section{Efficacy in maintenance treatment after an acute manic or depressed episode in preventing new mixed episodes}

Again, both extension studies (McIntyre et al. 2010a; Szegedi et al. 2012) do not supply separate data for mixed relapses after a manic index episode. A further double-blind, placebo controlled maintenance study (NCT01396291) has been presented at the 28th Annual United States Psychiatric and Mental Health Congress, September 10-13, 2015, San Diego, CA, USA, but has not been fully published yet. Patients were recruited while acutely manic or mixed according to DSM-IVTR criteria. After a 12-16 week open label run-in phase with asenapine, stable responder were randomized to the double- blind discontinuation phase over 26 weeks. Time to recurrence of any mood event during the double-blind period was significantly longer for asenapine than placebo. Time to recurrence for a mixed episode was numerically longer for asenapine but no test for significance has been reported. The number of patients with a mixed index episode was small $(n=55)$ and no sub-analysis for mixed patients at entry has been reported.

CE to prevent a mixed episode after a manic or depressed index episode (monotherapy) is „F”

CE to prevent a mixed episode after a manic or depressed index episode (combination/augmentation therapy) is „F”.

\section{Further evidence (FE)}

Combining both acute studies (McIntyre et al. 2009; McIntyre et al. 2010b) in a post hoc analysis, Azorin et al (Azorin et al. 2013) reported that the change in YMRS total score from baseline to week 3 was significantly greater $(p=0.015)$ with asenapine $(-15.0 \pm 0.9)$ compared to placebo $(-11.5 \pm 1.2)$. The difference between olanzapine and placebo, however, was not statistically different $(\mathrm{p}=0.169)$ on the mean YMRS total score change from baseline to week 
3 (-13.3 \pm 0.9$)$. The authors also report significant MADRS improvement with asenapine but not with olanzapine. However, the combined analysis was conducted using observed cases (OC), not with the ITT population. For this reason, and due to some inconsistencies and gaps in reporting, the task force decided to dismiss the combined analysis as a primary evidence ${ }^{4}$. A synopsis of clinical cases is in line with a good efficacy and safety profile of asenapine (Young et al. 2013). With the emergence of DSM-5, McIntyre et al (McIntyre et al. 2013) tested post hoc whether the positive findings for asenapine with respect to depressive symptoms in mixed mania still hold true when applying a proxy of the depressive mixed feature specifier by using MADRS or PANSS items. Of the 960 patients analysed from the two acute studies (McIntyre et al. 2008; McIntyre et al. 2010b), 34\%, 18\% and 4.3\% of patients, respectively, had $\geq 3$ depressive features with mild (score $\geq 1$ for MADRS items and $\geq 2$ for PANSS item), moderate (score $\geq 2$ MADRS, $\geq 3$ PANSS) and severe (score $\geq 3$ MADRS, $\geq 4$ PANSS) symptoms. In patients with $\geq 3$ depressive features and independent of treatment, MADRS remission (score $\leq 12$ ) rate decreased with increasing severity $(61-43 \%)$ and YMRS remission (score $\leq 12$ ) was similar for mild and moderate patients (36-37\%), but higher for severe (54\%). In asenapine-treated patients, the MADRS remission rate was stable regardless of baseline depressive symptom severity (range 64-67\%), whereas remission decreased with increasing severity with olanzapine (63-38\%) and placebo (49-25\%). Reduction in YMRS was significantly greater for asenapine compared with placebo at day 2 $(p \leq 0.01)$ across the three severity cut-offs and continued to decrease throughout the treatment period. These analyses confirm that depressive features are frequent in bipolar patients with manic episodes and have an impact on manic symptom remission rates. With increasing baseline severity of depressive features, treatment outcome was poorer with olanzapine and placebo, but remained stable with asenapine (McIntyre et al. 2013).

Considering long-term treatment, a pharmacoeconomic model was developed to simulate the management of Italian BD-I patients with mixed episodes over a 5-year time horizon by

\footnotetext{
${ }^{4}$ The Azorin et al 2013 paper states that „These post hoc efficacy and safety analyses were based on the intent-to-treat (ITT) dataset, which comprised all randomised patients who took at least one dose of study medication and had at least one valid post-baseline YMRS assessment ,, the ANCOVA of the YMRS and MADRS scores, however, were conducted on OC. Additionally, the paper does not supply the numbers of OC subject to analysis. Furthermore, the combined analysis reports on 295 ITT mixed patients, whereas adding the numbers of the individual studies leaves us with 302 ITT mixed patients, and the discrepancy between these numbers remains unclear. Due to these inconsistencies, the task force decided to dismiss the combined analysis as evidence in favour or against asenapine and olanzapine. In the opinion of the task force, this also makes the result for asenapine in the meta-analysis of Muralidharan et al (Muralidharan et al. 2013) questionable as the evidence for asenapine is only based on the publication by Azorin et al 2013, and, in addition, questions the validity of the whole meta-analysis which obviously mixes OC and LOCF data.
} 
combining clinical parameters with resource utilization (Caresano et al. 2014). An expert panel of Italian psychiatrists and health economists was responsible for adapting a UK model to the Italian context. The primary outcome measure of the economic evaluation was the incremental cost effectiveness ratio, where effectiveness is measured in terms of qualityadjusted life-years gained. Scenario analyses, sensitivity analyses, and a probabilistic sensitivity analysis were performed to test the robustness of the model.

This pharmacoeconomic model showed that asenapine was superior to olanzapine; in fact, asenapine was associated with lower direct costs (derived largely by the savings from hospitalizations avoided) and generated a better quality of life.

These findings were also confirmed by another health-economic study using a Markov-model and looking also at mixed patients (Sawyer et al. 2014).

Rating of FE: + for short-term treatment, + for long- term treatment

\section{Safety \&Tolerability (ST)}

The safety and tolerability profile of asenapine in short- and long-term treatment is generally considered as reasonably good, and especially better than, e.g., olanzapine with respect to metabolic issues (Vita et al. 2013) asenapine' s effects on weight and metabolic variables appear modest, as are its effects on the QTc interval and on prolactin (Citrome 2014). Asenapine has no appreciable affinity for muscarinic receptors and induces few anticholinergic side effects. In acute and continuation monotherapy, side effects occurring twice as frequently with asenapine as placebo (and in $>10 \%$ of subjects) included depression, dizziness, nausea, parkinsonism, tremor, and constipation (McIntyre et al. 2010a). Adverse effects reported in combination with lithium or valproate by $5 \%$ or more of patients and at twice the rate of placebo were sedation, somnolence, depression, constipation, oral hypoesthesia, irritability, and dyskinesia (Szegedi et al. 2012).

A brief note has to be made on the issue of practicability: asenapine is only as a sublingual formulation available. Intake and transient oral hypoesthesia as a possible side effect may constitute a problem in some patients.

Rating of ST: + for short-term treatment, + for long- term treatment

\section{Recommendation grade (RG)}

The $R G$ is „4” for the acute treatment of manic mixed episodes (for depressive symptoms only). 


\section{Carbamazepine}

\section{Efficacy in acute manic mixed episodes}

Two identically designed RCTs compared the acute efficacy of extended release Carbamazepine vs. placebo in DSM-IV acutely manic and mixed patients. The first study (Weisler et al. 2004) demonstrated significantly higher improvement of depressive symptoms in the subgroup of mixed patients for carbamazepine than placebo ( $\mathrm{p}=0.0003$ ), while no difference was observed for manic symptoms. The second study (Weisler et al. 2005) saw improvement for manic symptoms only $(\mathrm{p}<0.0001)$ but not for depressive symptoms $(\mathrm{p}=0.07)$. A combined analysis pooling the data from both trials was conducted and included 280 manic and 147 mixed patients. Unfortunately, the paper does not detail whether this was an a priori planned analysis as plan of the study protocols or post hoc- which, in this case, we have to assume. The improvement in manic and depressive symptoms was significant in the mixed subgroup of patients $(\mathrm{p}<0.01$ and $\mathrm{p}<0.05$, respectively), while in the case of pure manic patients this was only the case for manic but not depressive symptoms (Weisler et al. 2006).

In summary, we have two studies, one supporting antimanic efficacy and one supporting antidepressant efficacy in subgroup analyses of mixed patients, but at the same time supplying negative results for the respective opposite mood state. However, the positive pooled analysis supports efficacy both against manic and depressive symptoms, so a CE „C” can be justified.

We could not identify any acute combination treatment studies with carbamazepine in mixed patients fulfilling quality criteria.

$C E$ in acute manic mixed episodes (monotherapy) is "C” both for manic and depressive symptoms.

CE in acute manic mixed episodes (combination/augmentation therapy) is „ F”.

\section{Efficacy in acute depressive mixed episodes}

Although recommended as a third line monotherapy or together with lithium in a recent expert guideline on the management of mixed depression, we could not identify any published controlled study dedicated to carbamazepine in acute depressive mixed episodes. Dilsaver et al (Dilsaver et al. 1996) published a case series of carbamazepine monotherapy in 
bipolar depression, including nine patients with depressive mania (defined as meeting full criteria of mania and depression simultaneously). Improvement of depressive symptoms as measured with the HAM-D was the primary outcome- because of the chosen outcome measure and the context of the overall study (bipolar depression) we decided to count the study towards evidence for the treatment of acute depressive mixed episodes (and not mixed mania). The authors report significant improvement of depression with a mean reduction in HAM-D score in the course of treatment of $17.7 \pm 10.3(\mathrm{df}=8, \mathrm{t}=5.12, \mathrm{p}=.0009)$, and 2/9 patients entering remission after 2 weeks of treatment.

CE in acute depressive mixed episodes (monotherapy) is „C” for depressive symptoms.

CE in acute depressive mixed episodes (combination/augmentation therapy) is „F”.

\section{Efficacy in maintenance treatment after an acute mixed episode in preventing episodes of any polarity or a new manic, depressive, or mixed episode}

A 6-month, open-label study enrolled 92 patients with DSM-IV bipolar disorder (most recent episode: $67 \%$ [ $\mathrm{N}=62]$ mixed, $33 \%$ [ $\mathrm{N}=30]$ manic) who had participated previously in the two 3-week, double-blind, placebo-controlled studies (Weisler et al. 2004; Weisler et al. 2005). Extended release carbamazepine (200-1600 mg/day) was titrated at investigators' discretion to a final mean dose of $938 \mathrm{mg} /$ day. The primary efficacy measure was time to relapse, and secondary efficacy measures included Young Mania Rating Scale (YMRS), Clinical Global Impressions scale (CGI), and Hamilton Rating Scale for Depression (HAMD) scores. The authors reported long-term maintenance of the effect on depressive symptoms in the subgroup of mixed patients $(p=0.0003)$ when comparing baseline HAM-D scores of the double blind studies with endpoint scores of the present open-label study (Ketter et al. 2004). Unfortunately, no separate results for mixed patients were supplied for the other outcomes.

This finding is difficult to picture in our grading system. Maintaining low HAM-D scores is suggestive of prophylactic efficacy against new depressive or mixed depressive episodes, but not confirmative in the absence of reported numbers for relapses. Thus, the

CE to prevent a new episode after a mixed index episode (monotherapy and combination therapy) is „F”.

\section{Efficacy in maintenance treatment after an acute manic or depressed episode in preventing new mixed episodes}

A recent Swedish national registers study identified 35,182 individuals diagnosed with bipolar disorder. The registers provided information on lithium, valproate, carbamazepine, 
lamotrigine, quetiapine, and olanzapine treatment, as well as hospitalizations in psychiatric inpatient facilities between 2006 and 2009. 72.4\% were prescribed these drugs during the study period and $26.6 \%$ were hospitalized in inpatient psychiatric care. With each patient serving as his own control, there was no advantage of taking carbamazepine to prevent hospitalisation for a mixed episode (HR 1.65 (0.59-4.62)).

CE to prevent a mixed episode after a manic or depressed index episode (monotherapy) is „E”

CE to prevent a mixed episode after a manic or depressed index episode (combination/augmentation therapy) is „F”

\section{Further evidence (FE)}

A retrospective chart analyses in 22 RDC/DSMIII manic patients by Post et al (Post et al. 1989) supplied first evidence that carbamazepine might be a preferred treatment in mixed patients. Patients who responded better to carbamazepine ( $n=12$; improvement $>2$ points) were more severely manic at baseline, and tended to be more dysphoric (higher baseline ratings of depression) compared with seven non-responders. The degree of improvement in the dysphoric components of mania was highly correlated with the initial baseline severity of manic dysphoria, e.g. the highest depression ratings during mania, the greater improvement in depression $(\mathrm{p}<0.001)$.

In line with this, a randomized three-arm study from Iran reported significant efficacy of carbamazepine in reducing both manic and depressive symptoms in DSM-IV dysphoric mania (mania with 2-4 symptoms of MDD). Unfortunately, the paper is contradictory as far as the design of the study is concerned, at one occasion it says single-blind, at another double-blind. Additionally, it used comparators with unproven efficacy in mixed states: Gabapentin and lamotrigine, and numbers are small in each arm (Mokhber et al. 2008).

Findling and Ginsberg (Findling and Ginsberg 2014) conducted a 26 week open study of extended release carbamazepine in 157 children and adolescents suffering from a DSM-IV manic or mixed episode. Unfortunately, no separate outcomes are supplied for manic and mixed patients, but more than half of the participants were classified as mixed. At endpoint and compared to baseline, carbamazepine treatment resulted in a significant improvement in YMRS, Children's Depression Rating Scale, Revised (CDRS-R), the CGI-S, and the CGI Improvement (CGI-I). 
Rating of FE: + for short-term treatment, + for long- term treatment

\section{Safety \&Tolerability (ST)}

Common side effects of carbamazepine include over-sedation and blurred vision, especially with high dosages and rapid titration. Rare, but potentially severe side effects include allergic reactions, lupus erythematosus, agranulocytosis and hyponatremia. Tolerability issues may be less problematic with extended release formulations as used in the more recent studies. Carbamazepine is teratogenic with an estimated risk of neural tube defects of 0.5-1\%, and should be avoided during pregnancy (FDA pregnancy category „D”) (Ernst and Goldberg 2002). The use of carbamazepine can be complicated due to interaction with other psychotropic medication, including several antipsychotics, antidepressants and anticonvulsants (Spina et al. 1996), and auto-induction of its on metabolism may result in a constant adaption of dosage.

Rating of ST: 0 for short-term treatment, - for long- term treatment

\section{Recommendation grade (RG)}

The RG is „4” for the acute treatment of manic mixed episodes for manic and depressive symptoms.

The RG is „4” for the acute treatment of depressive mixed episodes for depressive symptoms.

\section{Cariprazine}

\section{Efficacy in acute manic mixed episodes}

Three double-blind, placebo controlled studies investigated the efficacy of cariprazine monotherapy in DSM-IV manic and mixed BD-I patients (Calabrese et al. 2015; Sachs et al. 2015; Durgam et al. 2015) that were also subject to a pooled analysis (Vieta et al. 2015). The Phase II study (Durgam et al. 2015) does not supply a separated subgroup analysis for mixed patients, probably because the numbers were too low (45 out of 236 patients) and only patients with mild depressive symptoms were allowed into the study. Of the phase III studies, none does supply any information (size, outcomes) about the mixed subgroup (Calabrese et al. 2015; Sachs et al. 2015), nor does the first pooled analysis of all studies. However, a more recent pooled analysis of the three studies looked into outcome by index episode. Of the 1037 patients in the ITT population, 14\% met criteria for mixed episodes (placebo=62, cariprazine $=83)$. The least squares mean difference $($ LSMD) for cariprazine versus placebo 
in YMRS total score change from baseline to week 3 was $-4.0(\mathrm{P}=.0254)$ in the mixed subgroup (Vieta et al. 2017).

$C E$ in acute manic mixed episodes (monotherapy) is „ $C$ ” for manic and „F” for depressive symptoms.

$C E$ in acute manic mixed episodes (combination/augmentation therapy) is „F”.

\section{Efficacy in acute depressive mixed episodes}

In an 8 week, placebo controlled, multinational phase II monotherapy study cariprazine 1.5 $\mathrm{mg} / \mathrm{d}$ demonstrated antidepressant efficacy compared to placebo. The YMRS was administered at baseline and throughout the study, but no information is supplied about baseline scores or whether there was a substantial subgroup with depressive mixed states included into the study.

A second Phase II monotherapy study (NCT00852202), conducted in the US, has been finished but so far, results have not been fully published. However, a poster presented by Yatham and colleagues at the 29th Annual U.S. Psychiatric \& Mental Health Congress, held October 21-24, 2016, in San Antonio, Texas report that the outcome was negative, most likely due to a high placebo response rate. No information on depressive mixed patients is supplied.

CE in acute depressive mixed episodes (monotherapy) is „F”.

CE in acute depressive mixed episodes (combination/augmentation therapy) is „F”.

Efficacy in maintenance treatment after an acute mixed episode in preventing episodes of any polarity or a new manic, depressive, or mixed episode

No maintenance data following a mixed index episode has been published so far.

CE to prevent a new episode after a mixed index episode (monotherapy): is „F”.

CE to prevent a new episode after a mixed index episode (combination/augmentation therapy): „F”

Efficacy in maintenance treatment after an acute manic or depressed episode in preventing new mixed episodes

No maintenance data for cariprazine in bipolar disorder has been published so far.

CE to prevent a mixed episode after a manic or depressed index episode (monotherapy) is „F” 
CE to prevent a mixed episode after a manic or depressed index episode (combination/augmentation therapy) is „F”

\section{Further evidence (FE)}

Rating of FE: 0 for short-term treatment, 0 for long-term treatment

\section{Safety \&Tolerability (ST)}

The most commonly reported adverse events in the acute trials (incidence $>5 \%$ and twice placebo) were extrapyramidal disorder, akathisia, vomiting, restlessness, somnolence, diarrhoea, blurred vision, and pyrexia. With the exception of akathisia and extrapyramidal disorder, the differences in incidence versus placebo for these events were generally small. The reported figures for akathisia range from $4.8 \%$ (for $1.5 \mathrm{mg}$ in bipolar depression to approx. 25\% with dosages up to $12 \mathrm{mg}$ in the acute mania studies (Citrome 2013; Durgam et al. 2016; Earley et al. 2017).

In long-term treatment, cariprazine shows some advantages compared to other atypical antipsychotics, especially the low propensity of weight gain, QTc Prolongation, prolactin elevation and metabolic abnormalities (Citrome 2013).

Rating of ST: + for short-term treatment, + for long- term treatment

\section{Recommendation grade (RG)}

The $R G$ is „4” for the acute treatment of manic mixed episodes for manic symptoms.

\section{Clozapine}

see „Other Atypical Antipsychotics used in Bipolar Disorder”

\section{Gabapentin}

see „Other Anticonvulsants used in Bipolar Disorder” 


\section{Lamotrigine}

\section{Efficacy in acute manic mixed episodes}

No randomized controlled studies in manic mixed patients nor subgroup analyses of studies in acute mania with lamotrigine have been reported.

$C E$ in acute manic mixed episodes (monotherapy) is " $F ”$.

$C E$ in acute manic mixed episodes (combination/augmentation therapy) is „F”.

\section{Efficacy in acute depressive mixed episodes}

No randomized controlled studies in depressive mixed patients nor subgroup analyses of studies in acute bipolar depression with lamotrigine have been reported. A multivariate analytical study was performed on two large depressed samples (one bipolar and the other MDD) that had been recruited for separate, contemporaneous, double-blind placebocontrolled trials of lamotrigine. The results suggest that that the clinical benefits of lamotrigine in acute bipolar depression are primarily upon depressive cognitions and psychomotor slowing, symptoms clearly not associated with mania.

CE in acute depressive mixed episodes (monotherapy) is „F”.

$C E$ in acute depressive mixed episodes (combination/augmentation therapy) is „F”.

Efficacy in maintenance treatment after an acute mixed episode in preventing episodes of any polarity or a new manic, depressive, or mixed episode

Two randomized, placebo and comparator (lithium) controlled studies over 18 months demonstrated the prophylactic efficacy of lamotrigine in BD-I patients, recently (hypo)manic or depressed (Calabrese et al. 2003; Bowden et al. 2003). The articles do not report on the inclusion of any mixed patients.

CE to prevent a new episode after a mixed index episode (monotherapy): is „F”.

CE to prevent a new episode after a mixed index episode (combination/augmentation therapy): „F”.

\section{Efficacy in maintenance treatment after an acute manic or depressed episode in preventing new mixed episodes}

In the study by Bowden et al, numerically more interventions for mixed episodes were needed in the placebo group $(n=6)$ than in the lamotrigine $(n=4)$ or lithium $(n=2)$ group. Unfortunately, neither the second study (Calabrese et al. 2003) nor a pooled analysis 
(Goodwin et al. 2004) supply additional separate data by medication for mixed recurrences. The Swedish registry study by Joas et al (Joas et al. 2017) could not establish a prophylactic effect of lamotrigine in preventing hospitalisation due to a mixed episode.

CE to prevent a mixed episode after a manic or depressed index episode (monotherapy) is „E“.

CE to prevent a mixed episode after a manic or depressed index episode (combination/augmentation therapy) is „F”.

\section{Further evidence (FE)}

The already mentioned randomized three-arm study from Iran (Mokhber et al. 2008) reported significant efficacy of lamotrigine in reducing both manic and depressive symptoms in DSMIV dysphoric mania comparable to what was seen with carbamazepine. However, as discussed, the limitations of this study appear too distinctive to count it as reliable evidence for lamotrigine in mixed mania.

Rating of FE: + for short-term treatment, 0 for long- term treatment

\section{Safety \&Tolerability (ST)}

In summary, the tolerability and long- term impact on weight and metabolic parameters of lamotrigine is good, but there are concerns with birth defects and allergic reactions. The incidence of a serious rash, however, appears low with the recommended slow titration scheme. Major congenital defects have been described with lamotrigine in $1.0 \%-5.6 \%$ of pregnancies. Despite an FDA pregnancy category „C“ rating, a teratogenic risk with lamotrigine treatment is suggested at doses exceeding $200 \mathrm{mg} /$ day (Morrow et al. 2006).

Rating of ST: + for short-term treatment, + for long- term treatment

\section{Recommendation grade (RG)}

none

\section{Lithium}

\section{Efficacy in acute manic mixed episodes}

Theoretically, lithium appears to be an effective acute treatment in mixed states, as it may alleviate both manic and depressive symptoms. However, the presence of a mixed state may be a strong predictor of poor response to lithium (Secunda et al. 1987; Swann et al. 1997). In 
a post hoc analysis of the acute mania study by Bowden et al (Bowden et al. 1994) there was no difference in treatment efficacy between lithium and placebo in the subgroup of patients with mixed mania in this randomized, double-blind study (Swann et al. 1997). The authors concluded that the presence of pre-treatment depressive symptoms is a predictor of nonresponse to lithium. However, as this was a post hoc analysis in a small number of patients, we would rather consider the evidence insufficient than negative. Unfortunately, the article does not supply any information on the effect on depressive symptoms.

Lithium or valproate have also been used as a basic treatment in add- on studies of different atypical antipsychotics vs. placebo, both in acute and maintenance treatment. The contribution of lithium to any improvement is impossible to grade, as there is no placebo comparison for the lithium treatment.

$C E$ in acute manic mixed episodes (monotherapy) is „F”.

CE in acute manic mixed episodes (combination/augmentation therapy) is „F”.

\section{Efficacy in acute depressive mixed episodes}

We could not identify any study of reasonable quality testing lithium in depressive mixed states

CE in acute depressive mixed episodes (monotherapy) is „F”.

CE in acute depressive mixed episodes (combination/augmentation therapy) is „F”.

\section{Efficacy in maintenance treatment after an acute mixed episode in preventing episodes of any polarity or a new manic, depressive, or mixed episode}

A retrospective study found that the presence of three symptoms of the opposite polarity was a predictor of poor long term outcome in lithium-treated mixed patients (Backlund et al. 2009). Observational maintenance studies with lithium also indicate that patients with an index episode of mixed mania were less likely to recover with long-term lithium treatment than were patients with an index episode of elated mania or bipolar depression (Keller et al. 1993). Sub-analysis of a randomized maintenance study comparing lithium, imipramine and the combination of the two revealed a higher rate of recurrences in mixed than pure manic patients (82\% versus 6\%) (Prien et al. 1988). However, as we have no comparison against placebo or an established effective prophylactic treatment for mixed states (which imipramine is not), the efficacy of lithium remains speculative based on these studies. 
On the other hand, the risk of re-hospitalization in patients with a mixed index episode was lower with lithium than with valproate as shown by a large Danish nationwide cohort study (Kessing et al. 2011b). Based on a post hoc analysis of the maintenance study by Bowden et al (Bowden et al. 2000) valproate was considered more effective than lithium as a prophylactic agent after a mixed index episode (Bowden et al. 2005) although neither medication separated from placebo. If we attribute a large register study a similar rigor of evidence as we do for a post hoc analysis of a RCT, we are left with conflicting evidence for the propensity of lithium to prevent a new episode after an index mixed episode. However, a planned secondary analysis of a large RCT over 104 weeks comparing the prophylactic efficacy of quetiapine and lithium vs. placebo supplies controlled evidence for lithium. Patients with a mixed index episode bear a significantly lower risk of a relapse into a new manic episode (HR (95\% CI) 0.34 (0.12-0.95)) and episode of any type compared to placebo (HR (95\% CI) 0.48 (0.27-0.86))(Weisler et al. 2011; Nolen and Weisler 2013).

CE to prevent a new episode after a mixed index episode (monotherapy): is „B” for a manic episode and „B” for any type.

CE to prevent a new episode after a mixed index episode (combination/augmentation therapy): „F”.

\section{Efficacy in maintenance treatment after an acute manic or depressed episode in preventing new mixed episodes}

We identified one controlled study reporting the prophylactic efficacy of lithium specifically against new mixed episodes. In a head-to head comparison, olanzapine $(n=217)$ was compared to lithium ( $\mathrm{n}=214$, target blood level: 0.6-1.2 $\mathrm{mmol} / \mathrm{l})$ in a double-blind, one year study in patients previously stabilized for 6-12 weeks on the combination of both agents while manic, and then randomized to continuation on either substance (Tohen et al. 2005). The primary outcome was testing non-inferiority of olanzapine against lithium for time to relapse/recurrence in the total population. Secondary results showed that compared with lithium, olanzapine had a significantly lower risk of symptomatic mixed episode relapse/recurrence (Tohen et al. 2016).

However, this result is at odds with a large analysis of the prophylactic efficacy of standard mood stabilizers using Swedish registry data (Joas et al. 2017). Whereas several drugs were efficacious in preventing manic or depressive recurrences, lithium and valproate were the only drug significantly associated with a reduced rate of admissions due to a mixed episode 
(Joas et al. 2017). Compared to other cohort studies, selection bias will be lower as each patient served as his own control comparing time periods on and off a specific medication; however, the order in which patients tried the respective medication could be a confounding factor. In a sensitivity analysis where the authors only included patients who had lithium prior to the other medications, the effect of lithium was attenuated whereas the effect of other medications was slightly enhanced. This suggests that patients who switched from lithium to a second drug were more likely to be non-responders to lithium.

CE to prevent a mixed episode after a unspecified index episode (monotherapy) is „D”.

CE to prevent a mixed episode after a manic or depressed index episode (combination/augmentation therapy) is „F”.

\section{Further evidence (FE)}

Kessing et al (Kessing et al. 2011a) compared rates of switch to, or add on of, another psychotropic, and rates of psychiatric hospitalization for patients treated with lamotrigine or lithium in clinical practice. From the Danish register they identified 730 patients who received lamotrigine and 3518 patients received lithium between 1995 and 2006. The overall rate of switch to or add on of another psychotropic was increased for lamotrigine compared with lithium (HR $=2.60,95 \%$ CI: 2.23-3.04), regardless of whether the index episode was depressive, manic, mixed or remission. In addition, the overall rate of psychiatric hospitalization was increased for lamotrigine compared with lithium (HR $=1.45,95 \% \mathrm{CI}$ : 1.28-1.65), as were the rates for patients with a depressive ( $\mathrm{HR}=1.31$, 95\% CI: 1.01-1.70) and patients with a manic ( $\mathrm{HR}=1.65,95 \% \mathrm{CI}$ : 1.31-2.09) index episode. Rates did not differ significantly between the drugs for patients with a mixed index episode and for patients in remission.

Another point which should be considered in clinical decision making is the high rate of suicidality and suicide attempts in mixed patients (Strakowski et al. 1996; Balazs et al. 2006). Lithium may exert a protective effect across the diagnostic spectrum in BD (Schaffer et al. 2015).

Rating for FE: 0 for short-term treatment, + for long- term treatment

\section{Safety \&Tolerability (ST)}

Side effects of lithium are well known and in their majority dependent on plasma level. Up to $75 \%$ of patients on lithium experience some side effects, but most are minor (transient 
metalic taste in mouth, polyuria, polydipsia, weight gain, mild oedema, concentration difficulties, sedation) and can be reduced or eliminated by dose adjustment or dosage schedule. Mild neurological symptoms with higher plasma levels of lithium are frequent.

From the patient perspective, in addition to the just mentioned adverse effects, the risk of weight gain and the risk of mental side effects (cognitive impairment and/or reduced intensity of perceptions and emotions) may be most crucial (Licht 2011).

Long term lithium treatment affects the kidney function (Tredget et al. 2010; Kessing et al. 2015), and close monitoring of the eGRF is essential part of lithium safety measures (Jefferson 2010). Hypothyroidism is frequent with lithium treatment, and substitution treatment is often indicated. Especially women seem to be on increased risk (women 14\% vs. men 4.5\%) (Johnston and Eagles 1999).

Lithium's teratogenic effect hardly ever gives rise to not initiating lithium treatment, possible because the risk is well characterized and relatively low in absolute terms.

Rating for ST: - for short-term treatment, - for long- term treatment

\section{Recommendation grade (RG)}

Based on the evidence, lithium monotherapy can be recommended to prevent a new manic or mood episode of any type after a mixed index episode ( $R G$ „3”). For the prevention of a mixed state after an unspecified index episode, the RG is “ 5 ”.

\section{Lurasidone}

\section{Efficacy in acute manic mixed episodes}

No randomized controlled studies testing the efficacy of lamotrigine in manic and mixed bipolar patients have been published.

CE in acute manic mixed episodes (mnontherapy and combination therapy) is „F”.

\section{Efficacy in acute depressive mixed episodes}

One placebo-controlled monotherapy (Loebel et al. 2014a) and one placebo-controlled combination treatment study (Loebel et al. 2014b) support the efficacy of lurasidone in acute bipolar depression. In a second controlled combination treatment trial lurasidone failed to separate from placebo-condition (Suppes et al. 2016a). 
Whereas the two combination treatment studies do not report on depressive mixed patient as a separate subgroup, the monotherapy study was subject to a post hoc analysis looking at patients with mixed manic features at study entry (McIntyre et al. 2015). At baseline, mixed features were present in 56\% of patients (lurasidone, $n=182 / 323$; placebo, $n=90 / 162$ ). Treatment with lurasidone (vs placebo) was associated with significantly greater reductions in MADRS scores in the mixed features group (-15.7 vs $-10.9 ; \mathrm{P}=.001$; week 6 ; MMRM analysis). Of note, rates of protocol-defined treatment-emergent hypomania or mania were similar for patients with mixed features (lurasidone, 2.2\%; placebo, 3.2\%) and without mixed features (lurasidone, 3.4\%; placebo, $0.0 \%$ ) suggestive of TEAS preventive capabilities of lurasidone.

CE in acute depressive mixed episodes (monotherapy) for depressive symptoms is „C”.

$C E$ in acute depressive mixed episodes (combination therapy) is „F”.

\section{Efficacy in maintenance treatment after an acute mixed episode in preventing episodes of any polarity or a new manic, depressive, or mixed episode}

One study (Calabrese et al, in press) investigated the efficacy of lurasidone vs placebo, both in combination with valproate or lithium, for preventing relapses over 28 weeks. The protocol followed an enriched design with patients being openly stabilised on lurasidone + lithium or valproate. The authors report on $29 \%$ less relapses in the lurasidone + lithium or valproate group compared to the placebo+ lithium or valproate group, (not significant). 84/496 randomized patients were classified as having a DSM-IV mixed state index episode; however, no separate outcome has been reported for this group.

Patients who completed the controlled acute treatment trials were also eligible for a 6-month open-label extension study (Ketter et al. 2016) which was then prolonged for an additional 18 months of continuation treatment with flexible, once-daily doses of lurasidone in the range of 20-80 mg (Pikalov et al. 2017). Improvement in depressive symptoms was maintained in the majority of patients treated with lurasidone, with relatively low rates of relapse, and with minimal effects on weight and metabolic parameters. Unfortunately, again no separate analysis for patients with a depressive mixed index episode are supplied.

CE to prevent any episode after a mixed index episode (monotherapy and combination therapy) is „F”.

\section{Efficacy in maintenance treatment after an acute manic or depressed episode in}




\section{preventing new mixed episodes}

In addition, the study of Calabrese et al. does also not differentiate between manic and mixed relapses in the total population (Calabrese et al, in press)

CE to prevent a mixed episode after a manic or depressed index episode (monotherapy and combination therapy) is „F”.

\section{Further evidence (FE)}

A small open, naturalistic, retrospective trial in BD-I and II patients was also supportive of antidepressant and long-term mood stabilizing properties of lurasidone add on to treatment as usual (TAU) (Schaffer et al. 2016). As previously mentioned, unipolar depression with mixed manic features is not within the scope of this guideline. Nevertheless, the positive result of a study with lurasidone in this patient group should be counted as supporting piece of evidence. In a study involving patients with major depressive disorder associated with subthreshold hypomanic symptoms (mixed features), lurasidone significantly improved depressive symptoms and overall illness severity, assessed by least squares mean change at week 6 in the MADRS and CGI-S scores: -20.5 compared with -13.0 (effect size, 0.80) and -1.8 compared with -1.2 (effect size, 0.60), respectively. Significant improvement in manic symptoms, assessed by the Young Mania Rating Scale, was also observed, in addition to other secondary efficacy endpoints (Suppes et al. 2016b).

Rating of FE: + for short-term treatment, 0 for long- term treatment

\section{Safety \&Tolerability (ST)}

TEAS reported in the lurasidone monotherapy study occurring with $>5 \%$ and more frequent than placebo were nausea, akathisia and sedation. Akathisia was also more as twice as frequent in mixed vs non-mixed bipolar depression (12.4\% vs $5.5 \%$ ) suggestive that true akathisia might have been be mingled with manic agitation. In the long- term study, there were no clinically meaningful, treatment-emergent differences between lurasidone and placebo in metabolic parameters as total cholesterol, HDL, LDL, triglycerides, glucose, or HbA1c. During the randomized study phase, there was also no difference in weight gain between study arms.

Rating of ST: + for short-term treatment, + for long- term treatment

\section{Recommendation grade (RG)}

In acute depressive mixed episodes, for depressive symptoms the RG is „, 4 ”. 


\section{Olanzapine}

\section{Efficacy in acute manic mixed episodes}

The efficacy of olanzapine as monotherapy was demonstrated in two consecutive short-term RCTs, which compared olanzapine efficacy vs. placebo in patients with DSM-IV criteria for manic and mixed episodes. In the first 3-week study (Tohen et al. 1999) conducted in 139 patients $(17.3 \%$ mixed), the olanzapine group showed significantly greater mean improvement in manic symptoms (YMRS score) in both pure and mixed manic patients, but there were no treatment advantages regarding improvement of depressive symptoms (HAMD score) in both subgroups. The second study, a 4-week RCT that recruited 115 patients (42.6\% mixed) (Tohen et al. 2000) confirmed the advantage of olanzapine vs. placebo on manic symptoms and response rate in both manic and mixed patients. In addition, and different from the first study, among those patients with moderate to severe depressive symptoms (HAM-D $\geq 20$ at baseline), there was a greater improvement in the olanzapine group in the reduction of ratings in depressive symptoms.

Two post hoc analyses looked into the pooled data of the above two RCTs: one confirmed that olanzapine was superior to placebo in improving both manic and depressive symptoms in both mixed ( $n=73$ ) and non-mixed patients ( $n=181)$ (Baldessarini et al. 2003). The other study (Baker et al. 2003) focused on those patients with moderate to severe depressive symptoms (or dysphoric, defined by a HAM-D score $\geq 20$ at baseline; $n=68$ ), and compared them against the non-dysphoric patients $(n=178)$. The study found that, while manic symptoms improved in both groups, depressive symptoms improved only in the dysphoric group $(\mathrm{p}=0.04)$, suggesting that olanzapine is effective for treating coexisting manic and depressive symptoms, especially when depressive symptoms are moderate to severe. A third post hoc analysis also including a Japanese study (Katagiri et al. 2012), looking at a total of 125 patients with mania with DSM-5 mixed depressive features specifier. Olanzapine was significantly more efficacious in patients both with and without DSM-5 mixed features compared to placebo, both in reducing YRMS and HAM-D scores; however, an even greater efficacy was observed in patients with DSM-5 mixed features (Tohen et al. 2014b). Finally, a post hoc analysis of the first acute RCT with asenapine (McIntyre et al. 2009) found that the subgroup of patients with a mixed episode at baseline $(n=150)$ had only a trend towards 
significant mean changes in manic symptoms with asenapine vs. placebo $(p=0.05)$, whereas olanzapine was superior to placebo $(\mathrm{p}<0.006)$.

There is also reasonable evidence for olanzapine as an add-on treatment in mixed states. Some studies have analysed the subgroup of mixed patients post hoc, or specifically recruited mixed patients. There is good evidence from two RCTs and one post hoc analysis. The first study, a 6-week RCT (Tohen et al. 2002), enrolled both DSM-IV manic and mixed patients (179 and 165, respectively), and compared the efficacy of olanzapine plus lithium/valproate vs. placebo plus lithium/valproate. Among patients with a current mixed episode, olanzapine co-therapy was superior to monotherapy in reducing the YMRS score from baseline (cotherapy: -12.92 ( \pm 8.37$), \mathrm{n}=121$; monotherapy: $-7.46( \pm 10.15), \mathrm{n}=54 ; \mathrm{p}<.001)$. The results showed that, among mixed patients, olanzapine was superior when adjunctive to valproate $(\mathrm{p}<0.0001)$, but not to lithium regarding manic symptoms, while there was no difference between lithium and valproate in pure manic patients. A subsample of patients with moderate to severe depressive symptoms was defined by having a current mixed episode and a HAM-D score $\geq 20$ at baseline. These patients exhibited better response and improvement in depressive symptoms in the olanzapine + lithium or valproate group. A secondary post hoc analysis (Baker et al. 2004) of this original study selected 85 patients with mania or mixed disorder that had substantial comorbid depression at baseline, also defined as a HAM-D score $\geq 20$ (dysphoric mania), and compared the efficacy of adjunctive olanzapine vs. nondysphoric manic patients. Dysphoric patients showed higher improvement of depressive symptoms than non-dysphoric patients did $(\mathrm{p}<0.001)$, while improvement in manic symptoms was independent of dysphoric/non-dysphoric categorization. Although the findings on improvement of manic symptoms appear valid, the reported higher improvement of depressive symptoms in dysphoric patients should be seen with caution, as baseline values are not comparable. Finally, a 6-week RCT, which specifically enrolled DSM-IV-TR mixed patients partially non- responsive to $\geq 14$ days of valproate monotherapy (Houston et al. 2009) showed that adjunctive olanzapine resulted in higher improvements in manic symptoms, depressive symptoms, or mania severity vs. valproate only $(p<0.001, p=0.022$, and $p=0.05$, respectively), and a shorter time to partial response and response with adjunctive olanzapine. This study is, so far, the only large placebo-controlled RCT that was especially designed and conducted for testing a medication in mixed states.

$C E$ in acute manic mixed episodes (monotherapy) is „ $A$ ” for manic and „ $C$ ” for depressive symptoms. 
$C E$ in acute manic mixed episodes (combination/augmentation therapy) is „ $B$ ” for manic and depressive symptoms.

\section{Efficacy in acute depressive mixed episodes}

A post hoc analysis (Benazzi et al. 2009) of an 8-week, double-blind trial of adult BD-I depression treated with placebo, olanzapine or OFC (Tohen et al. 2003b) found that patients treated with olanzapine showed a significantly higher response rate versus patients treated with placebo (OR = 1.95; 95\% CI, 1.14-3.34; $\mathrm{p}=.014$ ). This study, together with a second study in Japanese patients with bipolar depression (Tohen et al. 2012) was also subject to a pooled analysis (Tohen et al. 2014a). Patients with bipolar depression $(n=1214)$ were categorised according to the number of concurrent manic symptoms $(0,1,2$ or $\geq 3$, the latter being a proxy for DSM-5 mixed manic features specifier). Olanzapine was significantly better than placebo in reducing depressive symptoms, and no significant difference in MADRS response was observed between the four groups. The author concluded that olanzapine monotherapy was shown to be effective in the treatment of bipolar depression irrespective of the presence of concurrent manic symptoms.

In summary, we have two post hoc analyses showing efficacy for olanzapine in depressive mixed states. However, the patient sample overlaps in these two analyses, and it is not clear whether these analyses have been planned a priori as part of the protocol which would increase their value.

CE in acute depressive mixed episodes (monotherapy) for depressive symptoms is „C”.

CE in acute depressive mixed episodes (combination/augmentation therapy) is „F”.

\section{Efficacy in maintenance treatment after an acute mixed episode in preventing episodes of any polarity or a new manic, depressive, or mixed episode}

A RCT studied the efficacy of olanzapine vs. placebo as monotherapy for maintenance treatment in 361 patients with a DSM-IV diagnosis of manic or mixed episodes $(n=121$ mixed)(Tohen et al. 2006). In both patients with a manic or mixed index episode, the time to symptomatic relapse into any mood episode was significantly longer for patients who received olanzapine than for patients who received placebo $(\mathrm{p}<0.001)$. Furthermore, a post hoc analysis of this maintenance trial that focused on the subgroup of mixed patients (DSMIV definition) found that olanzapine-treated patients had significantly lower rates of symptomatic relapse of any kind. The median times to relapse of any kind was three times longer in the olanzapine group vs the placebo group $(\mathrm{p}<0.001)$. Olanzapine-treated patients 
also experienced longer time to depressive symptomatic relapse (85 versus 22 days, $\mathrm{p}=0.001$ ) and manic symptomatic relapse (too few relapses to calculate versus 42 days, $p<0.001$ ) than did placebo-treated patients (Tohen et al. 2009).

No firm data are available for maintenance treatment with olanzapine in combination with lithium or valproate. A randomized, placebo controlled study over 18 months included patients with a manic or mixed index episode (Tohen et al. 2004). No separate results for mixed patients or for relapses into mixed episodes are reported as the total number of patients entering the randomized maintenance phase was small $(n=99)$.

CE to prevent a new episode after a mixed index episode (monotherapy): is „B”.

CE to prevent a new episode after a mixed index episode (combination/augmentation therapy): „F”.

\section{Efficacy in maintenance treatment after an acute manic or depressed episode in preventing new mixed episodes}

We could not identify a placebo-controlled maintenance study analysing specifically prevention of mixed episodes. However, we found information potentially supportive for the use of olanzapine vs. lithium. A re-analysis of the one year maintenance study of olanzapine vs. lithium in recently manic or mixed patients (Tohen et al. 2005) utilising Multi-state Outcome Analysis of Treatments (MOAT) revealed that patients taking lithium spent significantly more time in mixed states than did patients taking olanzapine (Tohen et al. 2016). In contrast to this result, the Swedish registry study by Joas et al (Joas et al. 2017) could establish a prophylactic effect of lithium, but not olanzapine in preventing hospitalisation due to a mixed episode (HR for olanzapine: 0.78 (0.52-1.17)).

CE to prevent a mixed episode after a manic or depressed index episode (monotherapy) is „D“.

CE to prevent a mixed episode after a manic or depressed index episode (combination/augmentation therapy) is „F”

\section{Further evidence (FE)}

There are several more studies on the use of olanzapine as monotherapy, some of them performed in mixed/manic patients, some having analysed the subgroup of mixed patients post hoc. In 1999 a first case-report reported the short-term improvement in both manic and depressive symptoms of a patient with mixed BD treated with olanzapine monotherapy that 
was unresponsive to mood stabilizers and neuroleptics (Zullino and Baumann 1999). Olanzapine monotherapy vs. placebo has also been studied in the acute treatment of manic/mixed episodes in a population of adolescents. There were no significant differences for improvement of manic symptoms vs. placebo in neither manic nor mixed subgroups (Tohen et al. 2007). Finally, four RCTs have studied the efficacy of monotherapy olanzapine vs. an active comparator. The first one included manic and mixed patients and compared olanzapine vs. haloperidol; both therapies were equally effective in reducing manic and depressive symptoms (Tohen et al. 2003a). Another RCT compared the efficacy of olanzapine vs. risperidone in manic/mixed patients, and reported as well no significant differences in manic and depressive improvement, or rates of response or remission between treatments in neither manic nor mixed patients (Perlis et al. 2006). Finally, another RCT compared the efficacy of olanzapine vs. valproate or placebo in manic/mixed patients (Tohen et al. 2008). Both manic and mixed subgroups showed greater improvement in manic symptoms if treated with olanzapine $(\mathrm{p}=0.004)$. The first evidence for olanzapine-add on treatment came from a case report of two mixed bipolar patients who achieved complete remission of symptoms after the addition of olanzapine to mood stabilizers (Ketter et al. 1998). In the same line, an open-label study in nine patients with a mixed episode reported improvement in manic and depressive symptoms after the addition of olanzapine to mood stabilizers or neuroleptics (Sharma and Pistor 1999). An open-label study in rapid-cycling mixed patients reported that the addition of olanzapine to mood stabilizers resulted in a reduction of both manic and depressive symptoms in 10 out of the 13 enrolled subjects (76.9\%) (Gonzalez-Pinto et al. 2002). Moreover, a prospective open-label study over 12 months in manic/mixed patients compared subjects who received adjunctive or monotherapy olanzapine vs. those who did not have olanzapine. The likelihood of achieving remission for patients in the olanzapine group was significantly higher compared with patients in the nonolanzapine group for both patients with manic and mixed symptoms $(p=0.003$ and $p=0.2$, respectively) (Garcia-Bonetto et al. 2009).

Rating of FE: ++ for short-term treatment, + for long- term treatment

\section{Safety \&Tolerability (ST)}

The short-term and long term tolerability and safety profile has been described in great detail in previous publications (Grunze et al. 2009; Grunze et al. 2010; Grunze et al. 2013). Whereas short- term tolerability is reasonable, there are profound concerns about weight gain 
and long-term metabolic effects of olanzapine. For detailed information we refer the reader to the respective publications (Nasrallah and Newcomer 2004).

Rating for ST: + for short-term treatment, - for long- term treatment

\section{Recommendation grade (RG)}

The RG is " 2 " (monotherapy) for acute treatment in manic mixed states for manic symptoms and "4" for depressive symptoms, and " 3 " (combination treatment) for acute treatment in manic mixed states for manic and depressive symptoms. The RG is"4" (monotherapy) in depressive mixed states for depressive symptoms, RG “3” (monotherapy) for maintenance after a mixed index episode and RG “5” (monotherapy) to prevent a mixed state after an episode of mania or mixed mania.

\section{Oxcarbazepine}

see „Other Anticonvulsants used in Bipolar Disorder”

\section{Paliperidone}

\section{Efficacy in acute manic mixed episodes}

Positive evidence for the use of paliperidone monotherapy comes from two different 3-week RCTs. The first one compared the efficacy of extended-release (ER) paliperidone with quetiapine and placebo in 439 adult DSM-IV BD-I patients ( $n=268$ manic and $n=171$ mixed). Three weeks of ER-paliperidone was superior to placebo in manic symptom reductions, and non-inferior to quetiapine at 9-weeks follow-up, an effect not due to the baseline diagnosis of mixed or manic episode ( $\mathrm{p}=0.3$ )(Vieta et al. 2010). However, the article does not supply information whether quetiapine was superior to PLC in the mixed subgroup on its own. The other trial compared 3 different dosages of ER-paliperidone (3 mg, $6 \mathrm{mg}$, and $12 \mathrm{mg}$ ) vs. placebo in 469 patients with DSM-IV criteria for a manic or mixed episode ( $\mathrm{n}=163$ mixed). The highest dose was found to be superior in reducing manic symptoms to placebo in both the manic and mixed subgroup of patients $(\mathrm{p}=0.025)$, but not different from placebo in reducing depressive symptoms (Berwaerts et al. 2012b). However, in a further RCT patients with a mixed index episode had no profit from paliperidone treatment in combination with lithium or valproate (Berwaerts et al. 2011). A post hoc subgroup analysis by baseline 
diagnosis suggested that paliperidone + lithium or valproate treatment was superior to lithium or valproate monotherapy only for patients with a diagnosis of „acute manic episode“ in reducing manic (YMRS) scores $(\mathrm{p}=0.02)$, but not in mixed patients.

$C E$ in acute manic mixed episodes (monotherapy) is „ $B$ ” for manic and „E” for depressive symptoms.

CE in acute manic mixed episodes (combination/augmentation therapy) is „E”.

\section{Efficacy in acute depressive mixed episodes}

We could not identify any studies examining paliperidone in bipolar depressive mixed states.

CE in acute depressive mixed episodes (monotherapy) is „F”.

CE in acute depressive mixed episodes (combination/augmentation therapy) is „F”.

\section{Efficacy in maintenance treatment after an acute mixed episode in preventing episodes of any polarity or a new manic, depressive, or mixed episode}

We could not identify any studies examining paliperidone in maintenance treatment in patients with a mixed index episode and separate reporting of outcomes for mixed patients. The maintenance study by Berwaerts et al (Berwaerts et al. 2012a) only reports combined outcomes for manic and mixed patients.

CE to prevent a new episode after a mixed index episode (monotherapy): is „F”.

CE to prevent a new episode after a mixed index episode (combination/augmentation therapy): „F”.

\section{Efficacy in maintenance treatment after an acute manic or depressed episode in preventing new mixed episodes}

We could not identify any studies examining paliperidone in maintenance treatment reporting a separate outcome for newly emerging mixed episodes. In the study by Berwaerts et al (Berwaerts et al. 2012a) manic and mixed relapses are lumped together.

CE to prevent a mixed episode after a manic or depressed index episode (monotherapy) is „F”.

CE to prevent a mixed episode after a manic or depressed index episode (combination/augmentation therapy) is „F”. 


\section{Further evidence (FE)}

We could not identify any other relevant evidence for the use of paliperidone in BD-I patients with a mixed episode.

Rating of FE: 0 for short-term treatment, 0 for long-term treatment

\section{Safety \&Tolerability (ST)}

A meta-analysis including 15 studies with paliperidone, mostly in schizophrenia, found as adverse events with the greatest incidence extra-pyramidal symptoms (23\%), headache (14\%), insomnia (11\%), somnolence (9\%), tachycardia (9\%) and weight gain (8\%)(Harrington and English 2010). Short- and Long term safety and tolerability of paliperidone have also been described in a previous articles (Grunze et al. 2013). So far, there is little known about risks of paliperidone in pregnancy. It can be assumed that they may be similar to the parent substance, risperidone.

Rating of ST: 0 for short-term treatment, 0 for long- term treatment

\section{Recommendation grade (RG)}

Paliperidone is graded $R G, 3$ ” (monotherapy) for the acute treatment of manic mixed episode based on its efficacy against manic symptoms.

\section{Quetiapine}

\section{Efficacy in acute manic mixed episodes}

A three-week randomized, placebo-controlled trial investigated extended release quetiapine in manic or mixed episodes. For mixed patients (134 out of 316) as a subgroup, quetiapine was not better than placebo for improving of manic or depressive symptoms (Cutler et al. 2011). The study by Vieta et al (Vieta et al. 2010) where quetiapine served as an internal comparator for assay sensitivity unfortunately reports a separate sub-group analysis only for the paliperidone, but not the quetiapine patients.

As for its use as add-on therapy, there is a case report of a patient with mixed bipolar disorder with psychotic features not responding to the combination of valproate, olanzapine, and FX, who after the replacement of olanzapine by quetiapine improved in the manic and psychotics symptoms (Catapano-Friedman 2001). A retrospective study of patients with bipolar disorder and other bipolar spectrum disorders, reported that the proportion of mixed patients 
responding to quetiapine was 77\% (Zarate et al. 2000). Finally, there is a randomized controlled trial in hypomanic patients with mixed features (BD-II (DSM-IV-TR) with YMRS scores $\geq 12$ and MADRS scores $\geq 15$ ) that found that adjunctive quetiapine is superior to adjunctive placebo in improving overall severity as measured with the CGI-BP and depressive symptoms, but not (hypo)manic symptoms (Suppes et al. 2013).

$C E$ in acute manic mixed episodes (monotherapy) is „E” both for manic and depressive symptoms.

$C E$ in acute manic mixed episodes (combination/augmentation therapy) is „C” for manic symptoms and „B” for depressive symptoms.

\section{Efficacy in acute depressive mixed episodes}

We could not find any study investigating quetiapine monotherapy or adjunctive treatment in bipolar patients with a depressive mixed episode, only an add-on study in unipolar agitated depression (see „Further evidence”)

CE in acute depressive mixed episodes (monotherapy) is „F”.

CE in acute depressive mixed episodes (combination/augmentation therapy) is „F”.

\section{Efficacy in maintenance treatment after an acute mixed episode in preventing episodes of any polarity or a new manic, depressive, or mixed episode}

A large, placebo and lithium controlled relapse and recurrence prevention study with quetiapine included a subgroup of 223 randomized mixed patients who responded to quetiapine acutely during the open label run-in phase (Weisler et al. 2011). Quetiapine was significantly better than placebo in delaying time to recurrence for any mood episode (HR 0.26, 95\% CI 0.14-0.48), a new manic (HR 0.18, 95\%CI 0.06-0.53) and a new depressive episode (HR 0.32, 95\%CI 0.14-0.70). Unfortunately, new mixed episodes were not recorded as category of its own but summarized under new manic episodes.

We found two identically designed RCTs that studied maintenance treatment in mixed, manic, or depressive bipolar patients, and compared the efficacy of quetiapine+ lithium or valproate vs. placebo+ lithium or valproate (Vieta et al. 2008a; Suppes et al. 2009). Both studies found that, for the subgroup of mixed patients, the quetiapine combination increased the time to recurrence to a mood event (any, manic, or depressed). Furthermore, a post hoc analysis of the mixed patients included in these two previous RCTs confirmed these results, 
and further described that both manic and depressive symptoms improved with quetiapine combination ( $\mathrm{p}=0.004$ and $\mathrm{p}=0.011$, respectively) (Vieta et al. 2012).

CE to prevent a new episode after a mixed index episode (monotherapy): „B” for „any”, „B” for „manic” and „B” for „depressive”.

CE to prevent a new episode after a mixed index episode (combination/augmentation therapy): „A” for „any”, „A” for „manic” and „A” for „depressive” (all in combination with lithium or valproate),

\section{Efficacy in maintenance treatment after an acute manic or depressed episode in preventing new mixed episodes}

Unfortunately, the cited monotherapy and combination maintenance studies did not look into mixed recurrences as an outcome on its own. The Swedish registry study by Joas et al (Joas et al. 2017) could establish a prophylactic effect of lithium and valproate, but not quetiapine in preventing hospitalisation due to a mixed episode (HR for quetiapine: 0.92 (0.62-1.39)).

CE to prevent a mixed episode after a manic or depressed index episode (monotherapy) is „E”.

CE to prevent a mixed episode after a manic or depressed index episode (combination/augmentation therapy) is „F”

\section{Further evidence (FE)}

A RCT examined quetiapine add-on in 90 outpatients with BD-I or II, 10 of them with a mixed mood state, and current alcohol dependence. All primary outcomes were drink related. In the secondary outcomes, a non-significant greater reduction in the self- rated Inventory of Depressive Symptoms (IDS-SR) was observed with quetiapine; however, a separate analysis for mixed patients is not reported (Brown et al. 2014). Otherwise, we did not find any evidence for quetiapine in bipolar mixed episodes, however, some evidence in unipolar depression. A 6-weeks open-label study compared adjunctive quetiapine to venlafaxine in 21 patients experiencing acute agitated major depression to patients only taking venlafaxine as an antidepressant (Dannlowski et al. 2008). The decrease in depressive symptoms was markedly significant in the quetiapine group vs. venlafaxine monotherapy, being significant at week 1, and increased until week $6(\mathrm{p}=0.005)$; the remission rate was as well higher in the quetiapine group.

Rating of FE: + for short-term treatment, 0 for long-term treatment 


\section{Safety \&Tolerability (ST)}

The short term side effects of quetiapine include sedation, orthostatic hypotension, nausea, the long- term side effects weight gain and metabolic issues, although to a lesser degree as with, e.g., olanzapine. For more detailed information we refer the reader to the previous papers of this series (Grunze et al. 2009; Grunze et al. 2010; Grunze et al. 2013). Data on safety in pregnancy with quetiapine are sparse. Animal studies suggested that quetiapine may delay skeletal ossification as well as reduce birth weight (Nguyen et al. 2009) and as a consequence it is listed by the FDA as a category „C“ medication for safety in pregnancy.

Rating of ST: 0 for short-term treatment, - for long- term treatment

\section{Recommendation grade (RG)}

The RG is „3” (combination treatment) for the acute treatment of manic mixed episodes for depressive symptoms and " 4 " for manic symptoms.

The RG is „2" for prevention of any mood episode, mania and depression after a mixed manic index episode in combination with lithium or valproate, and „3” for prevention of any episode, mania, and depression, after a mixed manic episode, monotherapy.

\section{Risperidone}

\section{Efficacy in acute manic mixed episodes}

The only risperidone monotherapy trial including mixed patients did not show improvement of manic symptoms vs. placebo (Khanna et al. 2005). Changes at end-point in YMRS scores were significantly higher for risperidone only in the manic patients $(\mathrm{P}>0.001)$, but not significant for the mixed patients. However, only nine patients with mixed episodes were included in the study, so the result cannot be interpreted.

Best evidence for risperidone monotherapy stems from a three week, randomized and doubleblind head-to-head comparison of risperidone vs. olanzapine which can be considered as an efficacious and valid comparator or mixed episodes (Perlis et al. 2006). Of the 329 patients included, approximately 2/3 were diagnosed with a DSM-IV mixed episode. No significant differences in manic and depressive improvement, or rates of response or remission between treatments in neither manic nor mixed patients was found. The study was not powered to prove non-inferiority of risperidone compared to olanzapine. 
A controlled combination treatment study compared risperidone + lithium or valproate, haloperidol + lithium or valproate and placebo + lithium or valproate, including 97 mixed patients (Sachs et al. 2002). Patients with a mixed episode showed similar YMRS improvements with risperidone + lithium or valproate, haloperidol + lithium or valproate, and not different from improvement observed with placebo + lithium or valproate

CE in acute manic mixed episodes (monotherapy) is „C”.

CE in acute manic mixed episodes (combination/augmentation therapy) is „E”.

\section{Efficacy in acute depressive mixed episodes}

We could not identify any study with risperidone in patients with a bipolar depressive mixed episode.

CE in acute depressive mixed episodes (monotherapy) is „F”.

$C E$ in acute depressive mixed episodes (combination/augmentation therapy) is „F”.

\section{Efficacy in maintenance treatment after an acute mixed episode in preventing episodes of any polarity or a new manic, depressive, or mixed episode}

An open label 6- month study in 26 adult BD-I patients with a mixed index episode and additional treatment with lithium or valproate supplied some evidence for acute efficacy maintained for six months. Statistically significant reductions in YMRS score were seen at week $1(\mathrm{p}<0.005)$ and 6-month endpoint $(\mathrm{p}<0.0001)$. Highly significant improvements in CGI and PANSS score were seen from week 4 and onward $(\mathrm{p}<0.0001)$. At week $4,74 \%$ of patients were considered responders (50\% YMRS reduction and decrease of 2 points in CGI). Improvements in HRSD were significant from week 1 onward $(\mathrm{p}=0.0001)$ and remained significant until 6-months endpoint ( $\mathrm{p}<0.0001)$ (Benabarre et al. 2001).

A prospective open-label study from South Korea, examining adjunctive risperidone (plus mood stabilizer) in manic and mixed patients $(n=44)$ reported significant improvement of both manic and depressive symptoms in both subgroups over 24 weeks (Woo et al. 2010)

CE to prevent a new episode after a mixed index episode (monotherapy): is „F”.

CE to prevent a new episode after a mixed index episode (combination/augmentation therapy): „C”. 


\section{Efficacy in maintenance treatment after an acute manic or depressed episode in preventing new mixed episodes}

We could not identify any study with risperidone examining mixed relapses or recurrences

CE to prevent a mixed episode after a manic or depressed index episode (monotherapy) is „F”.

CE to prevent a mixed episode after a manic or depressed index episode (combination/augmentation therapy) is „F”.

\section{Further evidence (FE)}

An early case report was suggestive of efficacy of risperidone in treatment refractory dysphoric mania (Vieta et al. 1995).

Different from the outcome in the controlled study (Sachs et al. 2002), a 12 week, open label study including 102 patients, but only 17 with mixed episodes suggests efficacy for risperidone combined with lithium or valproate. Subjects with mixed episodes showed a significant reduction in mean YMRS score from baseline to week 1 ( $\mathrm{P}=0.0002)$, to week 3 ( $\mathrm{P}$ $=0.0001)$ and to week $12(\mathrm{P}<0.0001)$. The mean reductions in depressive symptoms scores were significantly reduced at week $3(\mathrm{P}<0.03)$ but not at week 12 in mixed patients (Yatham et al. 2003).

A retrospective chart review looking at 28 children and adolescent with DSM-IV bipolar disorder (25 mixed and 3 hypomanic) supports efficacy of risperidone in reducing manic and psychotic symptoms (Frazier et al. 1999).

Rating of FE: + for short-term treatment, 0 for long-term treatment

\section{Safety \&Tolerability (ST)}

The safety and tolerability profile of risperidone has been described in great detail in previous articles of this series (Grunze et al. 2009; Grunze et al. 2013) and in key review papers (Seemüller et al. 2005). In brief, extrapyramidal symptoms (especially with higher dosages), akathisia and insomnia in short-term and weight gain and prolactin elevation in long-term treatment may constitute problems.

The FDA safety -in -pregnancy category rating for risperidone is „C”.

Rating of ST: 0 for short-term treatment, - for long- term treatment 


\section{Recommendation grade (RG)}

The RG is „4" for the acute treatment of manic mixed episodes (Monotherapy)

The RG is "4" to prevent a new episode after a mixed index episode (combination/ augmentation therapy)

\section{Typical antipsychotics (first generation antipsychotics)}

\section{Efficacy in acute manic mixed episodes}

A controlled 12 week head-to head comparison of olanzapine and haloperidol (Tohen et al. 2003a) included a small subgroup of 24 mixed patients. Rates of symptomatic remission (YMRS scores) were not different between olanzapine and haloperidol groups, and there was no significant interaction for the treatment by manic or mixed subtypes. Secondary analysis revealed that for patients who entered the study with a mixed state both therapies were effective in reducing depressive symptoms.

A controlled combination treatment study compared risperidone + lithium or valproate, haloperidol + lithium or valproate and placebo + lithium or valproate, including 97 mixed patients (Sachs et al. 2002). Patients with a mixed episode showed similar YMRS improvements with risperidone + lithium or valproate, haloperidol + lithium or valproate not different from improvement observed with placebo + lithium or valproate.

CE in acute manic mixed episodes (monotherapy) is „C”.

$C E$ in acute manic mixed episodes (combination/augmentation therapy) is „E”.

\section{Efficacy in acute depressive mixed episodes}

We could not identify any study with a typical antipsychotic in patients with a bipolar depressive mixed episode.

$C E$ in acute depressive mixed episodes (monotherapy) is „F”.

$C E$ in acute depressive mixed episodes (combination/augmentation therapy) is „F”.

Efficacy in maintenance treatment after an acute mixed episode in preventing episodes of any polarity or a new manic, depressive, or mixed episode

We could not identify any maintenance study with a typical antipsychotic in patients with a bipolar mixed index episode. 
CE to prevent a new episode after a mixed index episode (monotherapy): is „F”.

CE to prevent a new episode after a mixed index episode (combination/augmentation therapy): „F”.

\section{Efficacy in maintenance treatment after an acute manic or depressed episode in preventing new mixed episodes}

We could not identify any study with typical antipsychotics examining mixed relapses or recurrences.

CE to prevent a mixed episode after a manic or depressed index episode (monotherapy) is „F”.

CE to prevent a mixed episode after a manic or depressed index episode (combination/augmentation therapy) is „F”.

\section{Further evidence (FE)}

No further studies of typical antipsychotics in mixed states have been identified

Rating of FE: 0 for short-term treatment, 0 for long-term treatment

\section{Safety \&Tolerability (ST)}

Similar as with antidepressants we are not looking into a homogenous group of medications. The safety and tolerability profile varies. The use of most typical antipsychotics is associated with extrapyramidal motor symptoms both in the short and long term, with tardive dyskinesias and probably CNS neurotoxic effects in the long run, as well as with differing degrees of prolactin elevation and weight gain. As far as weight gain is concerned, some typical AP are by large weight neutral, such as molindone, fluphenazine, perphenazine, pimozide or haloperidol, others may cause significant weight gain, e.g. chlorpromazine. Finally, typical antipsychotics put patients on greater risk of a malignant neuroleptic syndrome than atypical antipsychotics (Tural and Onder 2010)

The risk of major congenital malformations in pregnancy might differ between agents. Haloperidol is generally considered as a relatively safe option (Diav-Citrin et al. 2005)

Rating of ST: - for short-term treatment, - for long- term treatment

\section{Recommendation grade (RG)}

The $R G$ is „4" for the acute treatment of manic mixed episodes (monotherapy with haloperidol). 


\section{Valproate (incl. Divalproate, Divalproex, Valpromide)}

\section{Efficacy in acute manic mixed episodes}

In contrast to several evidence-based recommendations we could not identify a placebocontrolled randomized study that proves efficacy of valproate in mixed patients. Subgroup analyses in mixed patients have either not been conducted/reported (Freeman et al. 2002; Bowden et al. 2006) or did not differentiate from placebo (Swann et al. 1997). Swann et al reported on a post hoc analysis of a 3-week RCT (Bowden et al. 1994) that enrolled 179 adult patients with Research Diagnostic Criteria (RDC) criteria for acute mania. 103 patients also met mixed mania criteria (or, as the authors named it, “depressive mania”) defined as the presence of least two items of the Schedule for Affective Disorder and Schizophrenia Change [SADS-C] depression subscale. The study found that patients with depressive mood improved more in their manic symptoms when treated with valproate than with lithium $(\mathrm{p}<0.005)$. The study also demonstrated that presence of even a modest level of pre-treatment depressive symptomatology was indicative of a superior response to valproate over lithium. However, this finding has to be seen with big caution as numbers of patients with mixed mania were small, valproate did not differentiate from placebo on a significant level in the subgroup with mixed mania (only from lithium) and the analysis was post hoc, and from today's point of view lithium would not be considered as a gold standard for acute mixed states against which new medications would be tested for relative efficacy.

A small case series tested valproate as intravenous infusion in seven severely manic, mixed or bipolar depressed patients (two manic, two mixed, one mixed with RC, two depressed). 4/7 patients were classified as responders as far as a reduction of manic symptoms and overall improvement are concerned, only the RC patient and the two depressed patients did not improve (Grunze et al. 1999).

CE in acute manic mixed episodes (monotherapy) is „C” for manic symptoms.

CE in acute manic mixed episodes (combination/augmentation therapy) is „F”.

\section{Efficacy in acute depressive mixed episodes}

We could not identify any study with valproate in patients with a bipolar depressive mixed episode.

CE in acute depressive mixed episodes (monotherapy) is „F”. 
$C E$ in acute depressive mixed episodes (combination/augmentation therapy) is „F”.

\section{Efficacy in maintenance treatment after an acute mixed episode in preventing episodes of any polarity or a new manic, depressive, or mixed episode}

A post hoc analysis of the 12-month maintenance study comparing valproate, Lithium and placebo (Bowden et al. 2000) has been published in 2005 (Bowden et al. 2005). Of 372 patients, 123 were classified as having dysphoric mania (mania $+\geq 2$ depressive symptoms). Compared to placebo, there were no significant treatment-related differences in the dysphoric patients on time to a depressive or manic episode. Among both euphoric and dysphoric patients, maintenance treatment with valproate was superior to lithium (but not placebo) in delaying time to any mood episode or premature discontinuation. Dysphoric mania appeared to predispose patients to more side effects when treated with either valproate or lithium compared to placebo treatment.

Kessing et al (Kessing et al. 2011b) conducted an observational cohort study with linkage of nationwide registers of all people with a diagnosis of bipolar disorder in psychiatric hospital settings who were prescribed valproate or lithium in Denmark between 1995 and 2006. They included a total of 4268 participants among whom 719 received valproate and 3549 received lithium subsequent to the diagnosis of bipolar disorder. The overall rate of hospital admissions was significantly increased for valproate compared with lithium in patients with a mixed index episode ( $\mathrm{HR}=1.59,95 \%$ CI 1.16-2.18), which is in contrast to previous reports suggestive of better outcomes with valproate than lithium maintenance.

CE to prevent a new episode after a mixed index episode (monotherapy): is „E”.

CE to prevent a new episode after a mixed index episode (combination/augmentation therapy): „F”.

\section{Efficacy in maintenance treatment after an acute manic or depressed episode in preventing new mixed episodes}

The already cited Swedish registry study by Joas et al (Joas et al. 2017) showed that valproate was beside lithium the only drug that significantly reduced mixed relapses. As detailed previously, we consider this registry study due to its size and quasi-experimental design minimizing selection bias as evidence sufficient to qualify for a CE „B“.

In a 20-month maintenance RCT comparing valproate and lithium in recently manic RC patients who were initially stabilized on combined treatment with lithium and valproate (Calabrese et al. 2005) no statistical difference emerged between valproate and lithium in 
preventing a mixed episode. However, the number of mixed recurrences was low: 0 on valproate and 1 on lithium in altogether 60 patients (Cipriani et al. 2013). In addition, the study is difficult to interpret as it has no placebo arm, analysis was post hoc for the purpose of a meta-analysis, and lithium may be not the ideal standard comparator.

CE to prevent a mixed episode after an unspecified, a manic or depressed index episode (monotherapy) is „B”.

CE to prevent a mixed episode after a manic or depressed index episode (combination/augmentation therapy) is „F”.

\section{Further evidence (FE)}

The study by Freeman et al (Freeman et al. 1992) was the first controlled study that indicated that valproate was effective in the treatment of depressive symptoms that occurred concomitantly in patients with mania.

A single case report suggest efficacy in an adolescent patient with dysphoric mania and learning disability (Whittier et al. 1995)

Mixed features appear frequent in geriatric patients with bipolar disorder (Sajatovic et al. 2011). A open study by Niedermier (Niedermier and Nasrallah 1998) is suggestive of efficacy of valproate in this patient group.

A 3-week RCT study, recruiting DSM-IV-TR mixed or manic patients ( $\mathrm{n}=166$ mixed, 45 \% of total study population) showed that extended release Val was superior to placebo in improving manic scores $(p=0.13)$ and response $(p=0.012)$. The article states that the treatment difference that was independent of presenting a manic or a mixed episode, however, a separate subanalysis for mixed patients is not supplied. (Bowden et al. 2006)

Rating of FE: + for short-term treatment, 0 for long-term treatment

\section{Safety \&Tolerability (ST)}

Usually valproate is well tolerated but may give rise to potentially life- threatening problems. More frequent dose dependent acute side effects include neurological symptoms such as tremor and mild sedation, thrombopenia or leukopenia and asymptomatic increase of liver transaminases. These side effects are usually benign and fully reversible after discontinuation of valproate. Hair loss or change of hair texture may occur. Of the severe and potentially life threatening adverse events, idiosyncratic hepatic failure occurs in approximately 1 in 50,000 
patients with valproate and is not dose dependent. Acute haemorrhagic pancreatitis with valproate has been observed in a few cases and occurs most likely in the first 3 months of treatment. Valproate- induced encephalopathies are described in epilepsy treatment.

Weight gain is probably the most prominent side effect in long- term treatment and may affect medication adherence. Polycystic ovary syndrome (PCOS) in valproate treated female patients is also an important issue, and together with valproate's teratogenicity (FDA pregnancy category D) makes it use not suitable in young women of child- bearing age. Valproate is associated with the highest rate of major congenital malformations (6.2\%-16\%) (Nguyen et al. 2009). In addition, lasting developmental delays of children of mothers who had taken valproate during pregnancy has been described (Meador et al. 2009).

Rating of ST: + for short-term treatment, - for long-term treatment

\section{Recommendation grade (RG)}

The RG is „4” (monotherapy) for the acute treatment of manic mixed episodes

The $R G$ is „3” for preventing a mixed episode after an manic, mixed or unspecified index episode.

\section{Ziprasidone}

\section{Efficacy in acute manic mixed episodes}

Two RCTs showing the efficacy of ziprasidone in acute treatment of mixed states used monotherapy. The first study is a 3-week RCT including both DSM-IV manic and mixed patients with BD-I disorder (BPI) ( $n=127$ manic, and $n=70$ mixed) (Keck et al. 2003b). The results of this trial showed that ziprasidone was superior to placebo in improving manic symptoms (decrease in Mania Rating Scale [MRS] scores) and mania severity (Clinical Global Impression - Severity scale [CGI-S]), being associated with a higher rate of responders that were comparable in both manic and mixed subsets of patients. However, the paper unfortunately does not supply information on the significance of these findings in the respective subgroups. The replication trial by Potkin et al (Potkin et al. 2005) included 83 mixed patients, but did not report a separate subgroup analysis. So the controlled evidence rests with a post hoc pooled analysis of these short-term studies that re-examined the subgroup of 179 patients with subsyndromal depressive symptomatology (Cincinnati Criteria, or experiencing $\geq 2$ prominent depressive symptoms)(Stahl et al. 2010). The analysis showed 
that improvement in manic symptomatology was greater in the ziprasidone group $(\mathrm{p}<0.001)$, and depressive symptoms were significantly lower at all visits $(\mathrm{p}<0.05)$, with higher response and remission rates than placebo.

CE in acute manic mixed episodes (monotherapy) is „ $C$ ” both for manic and depressive symptoms.

CE in acute manic mixed episodes (combination/augmentation therapy) is „F”.

\section{Efficacy in acute depressive mixed episodes}

A 6-week, randomized, placebo-controlled trial enrolled 73 patients with BD-II disorder $(n=43)$ or major depressive disorder (MDD) who met DSM-IV criteria for a major depressive episode (MDE) together with 2 or 3 DSM-IV mania criteria (Patkar et al. 2012). Patients were maintained on their ongoing TAU while ziprasidone was added. The study reported that both mixed BD-II and MDD patients on ziprasidone had higher response and remission rates ( $p=0.04$ and $p=0.0045$, respectively); manic symptoms (MRS scores) did not change over time, but there was a reduction in depressive symptoms (MADRS scores; $\mathrm{p}=0.001$ ) vs. placebo. The effect of ziprasidone on MADRS scores was significantly different in the two diagnoses subtypes ( $p=0.036)$, with more benefit in type II bipolar disorder than MDD.

The same study was also subject to an earlier report (Pae et al. 2012) that did not find any significant effects. In this earlier report, statistical significance was conservatively set at a pvalue $\leq 0.003$ to detect at least a medium- large effect size. The power analysis in the second report ((Patkar et al. 2012) set a less ambitious $\beta=0.20$ and two-tailed $\alpha=0.05$ based on pilot studies previously conducted for the mania registration trials. In this case of double reporting the task force decided to go along with the report by Patkar et al 2012 using standard significance levels.

CE in acute depressive mixed episodes (monotherapy) is „F”.

$C E$ in acute depressive mixed episodes (combination/augmentation therapy to TAU) is „B” for depressive symptoms.

\section{Efficacy in maintenance treatment after an acute mixed episode in preventing episodes of any polarity or a new manic, depressive, or mixed episode}

Sixty-two patients, 19 with a DSM-IV mixed index episode, participated in a one year extension after participating in the previously described acute study by Keck et al (Keck et al. 2003b) and having received ziprasidone in this study. The mean reduction in the MRS as well 
as CGI-S ratings, compared to baseline of the acute study, were sustained in the mixed subgroup. The overall MRS response rate was $86 \%$ (88\% in the manic, $79 \%$ in the mixed subgroup) (Keck et al. 2009b). Unfortunately, measurements of depressive symptoms are not reported. Only 17 patients finished the study after one year, which makes the results less reliable and warrants further investigations.

Another study investigated the prophylactic efficacy of ziprasidone plus lithium or valproate in a randomised, placebo-controlled design in 240 patients who had recovered from a manic (56\%) or mixed (44\%) index episode. The overall result was that add-on ziprasidone resulted in a significantly longer time to intervention than placebo; unfortunately, no detailed outcome for those with mixed episodes at entry nor the numbers for mixed recurrences are supplied (Bowden et al. 2010).

CE to prevent a new episode after a mixed index episode (monotherapy) is „C” (for manic relapse).

CE to prevent a new episode after a mixed index episode (combination/augmentation therapy) is „F”.

\section{Efficacy in maintenance treatment after an acute manic or depressed episode in preventing new mixed episodes}

We could not identify any study with ziprasidone examining mixed relapses or recurrences

CE to prevent a mixed episode after a manic or depressed index episode (monotherapy) is „F”.

CE to prevent a mixed episode after a manic or depressed index episode (combination/augmentation therapy) is „F”.

\section{Further evidence (FE)}

A case report by Mech (Mech 2008) reports good efficacy in 3 out of 4 treatment refractory mixed patients, both for manic and depressive symptoms.

Rating of FE: + for short-term treatment, 0 for long-term treatment

\section{Safety \&Tolerability (ST)}

The safety and tolerability profile of ziprasidone appears favourable (Citrome 2011). Of note is the negligible impact on metabolic parameters, prolactin and the fact that ziprasidone is relatively weight neutral (Kemp et al. 2012). Some sedation, although less than with several 
other APs, and EPS, especially tremor and akathisia, are more frequent side effects (Seemüller et al. 2005). Although ziprasidone can prolong the QTc interval, this has not resulted in increases in sudden death or cardiac sudden death (Camm et al. 2012). Ziprasidone is in the FDA „C” pregnancy category meaning that risk cannot be ruled out as there are no controlled data in human pregnancy, but animal studies have revealed evidence of developmental toxicity including possible teratogenic effects, an increase in the number of offspring born dead, and a decrease in postnatal survival. However, a developmental delay after in -utero- exposure has been observed for ziprasidone in a preliminary report, so for now, ziprasidone should be used even more cautiously in pregnancy than other antipsychotics (Nguyen et al. 2009).

Rating of ST: + for short-term treatment, + for long- term treatment

\section{Recommendation grade (RG)}

The $R G$ is „3” (monotherapy) for the acute treatment of manic mixed episodes for manic and depressive symptoms.

The $R G$ is „3” (combination treatment) for the acute treatment of depressive mixed episodes for depressive symptoms.

The RG is „4” (monotherapy) for prevention of mania after a mixed manic index episode

\section{Other Atypical Antipsychotics used in Bipolar Disorder}

We identified two small studies on the use of clozapine in mixed state. A retrospective chart review examined clozapine in dysphoric manic patients (according to Cincinnati criteria (McElroy et al. 1992)) as monotherapy or combined with lithium, valproate, or an antidepressant. All 7 patients improved significantly both in their psychotic and affective symptoms (Suppes et al. 1992). Further evidence comes from another study that was openlabel, and enrolled 10 adolescents with treatment resistant manic/mixed episodes. With clozapine alone or in combination with mood stabilizers all patients (5 pure manic, 5 mixed) were considered responders, and with significant improvements in manic and depressive outcomes (all $\mathrm{p}<0.001$ ) (Masi et al. 2002). Issues exist with safety (especially agranulocytosis) making frequent blood check mandatory (ST“_“). In summary, we would consider for clozapine a $C E$ „C” evidence for acute manic mixed episodes (mono- and combination/augmentation therapy). 
We could not identify any published studies for other atypical antipsychotics used occasionally in mania, namely amisulpride, sulpiride or zotepine supporting their use in bipolar mixed states.

\section{Other Anticonvulsants used in Bipolar Disorder}

Oxcarbazepine has been infrequently used in bipolar patients as alternative to carbamazepine in patients not tolerating carbamazepine well or in need of co-medication that strongly interferes with carbamazepine. However, also oxcarbazepine has an interaction potential with other medication, and the risk of hyponatremia might me higher than with carbamazepine (Van Amelsvoort et al. 1994). There is some evidence from an open 8 week add-on study by Benedetti et al that oxcarbazepine has some efficacy in DSM-IV mixed manic states (Benedetti et al. 2004). Eighteen adult patients with BD-I $(n=16)$ and BD-II $(n=2)$ disorder with a DSM-IV diagnosis of bipolar manic $(n=4)$, depressive $(n=8)$ or mixed episode $(n=6)$, and unsatisfactory clinical response to current treatment with lithium salts administered for at least one month received add-on oxcarbazepine. Five out of 6 mixed patients were classified as responders (CGI-I score of 2 or 1 at week 8). Thus, we would consider it as a $C E, C$ „ evidence for acute manic mixed episodes (combination/augmentation therapy).

The already detailed 8-weeks controlled monotherapy study in dysphoric mania by Mokhber et al (Mokhber et al. 2008) found that gabapentin is superior to carbamazepine in mania ratings, and better than lamotrigine and carbamazepine in depression ratings. However, the already discussed shortcoming in design and reporting makes it difficult to consider this study. As adjunctive to mood stabilizers therapy, gabapentin has been studied in five small sized open-label studies. In one of them, 8/9 Bipolar I or II patients with mixed symptoms inadequately responsive to mood stabilizers, improved in their manic symptoms in the short term (1-3 months), and maintained response (1-7 months) (McElroy et al. 1997). In another one, which recruited 21 patients with BD-I mixed episode, found that 50\% of them were responders (CGI 1 or 2 points), and that while there was no significant reduction of manic symptoms, depressive symptoms were greatly reduced $(\mathrm{p}=0.0001)$, a reduction positively correlated with mixed residual symptomatology $(p=0.003$ ) (Perugi et al. 1999). In another study on BDI or BDII patients inadequately responding to standard therapy, 5 out of the 9 mixed patients included had a positive response although lower and with a time to response longer than for manic or hypomanic patients (Altshuler et al. 1999). Another one enrolled 10 mixed bipolar patients who improved quickly in both manic $(\mathrm{p}<0.01)$ and depressive 
( $\mathrm{p}<0.05)$ symptoms when GBP was added (Sokolski et al. 1999). Finally, another open-label study which enrolled BDI or II patients with subsyndromal features (YMRS>6 or HDRS>12, and CGI-B>3) found again that while mania scores did not substantially improve, depression was significantly improve $(\mathrm{p}<0.002)$ (Vieta et al. 2000). Thus, we would consider it as a $C E$ „C” evidence for acute manic mixed episodes, both for manic and depressive symptoms (combination/augmentation therapy).

Topiramate has been tested in 5 RCT's of acute mania, but failed to separate from placebo and was inferior to lithium (Grunze et al. 2009; Pigott et al. 2016). None of these studies supplied a subgroup analysis for patients with mixed states, so evidence for topiramate derives only from one retrospective chart review and two open studies that used it as adjunctive therapy in patients refractory to other treatments. In the retrospective chart review which included 58 patients with bipolar or schizoaffective disorder refractory to mood stabilizer treatment, 3 out of the 7 patients with a mixed affective disorder (DSM-IV criteria), showed marked improvement (42\%), while the remaining patients showed mild or no improvement (29\%), or deteriorated (28\%) (Marcotte 1998). The open-label study by Chengappa et al (Chengappa et al. 1999) also included patients resistant to mood stabilizer or antipsychotic treatment; out of the five mixed patients included, three (60\%) responded with regards to a reduction in manic and depressive symptoms. The other open-label study, which recruited bipolar spectrum patients, found that out of the three mixed subjects one was responding (50\% decrease in both manic and depressive symptoms), while two had a depressive episode relapse and one a mixed relapse (Vieta et al. 2002). A retrospective study in adolescents evaluated adjunctive topiramate, and found that $59 \%$ of patients with mixed mania (10 out of 17) responded to treatment (CGI-I $\leq 2)$ (Barzman et al. 2005).

The safety/tolerability profile of topiramate appears reasonable in low doses commonly used in bipolar disorder (rating „,”), however, neurological side effects are not entirely dosedependent, including cognitive impairment and rare cases of transient hemiparesis (Jones 1998).

In summary, the magnitude of change and thus the efficacy of topiramate in mixed states appears not convincing and may just resemble spontaneous improvement and placebo effects. We consider the CE for topiramate added to ongoing treatment in acute mixed states conflicting (CE „D”), the corresponding RG „,5“. 
We could not identify any published studies for other anticonvulsants sometimes used in bipolar disorder, namely clonazepam, phenytoin, pregabalin, zonisamide, eslicarbazepine, levetiracetam, barbiturates and bromides supporting their use in bipolar mixed states. However, our search may have missed evidence, especially for the first generation antiepileptics, as it may have been published prior to the inclusion period of our literature search.

\section{Other medication tested in bipolar mixed states}

Burt et al (Burt et al. 1999) conducted an open label trial with the cholinesterase inhibitor donepezil add-on in 11 adult patients with a diagnosis of BD-I disorder partially or nonresponsive to Lithium. Of the five mixed patients, two showed a marked and one a slight response. Available evidence for the calcium channel blocker verapamil comes from two open-label studies. In the first one, verapamil (with adjunctive chlorpromazine if necessary) was tested in mania with mood congruent or mood incongruent psychotic features, or mixed episodes. The two mixed patients in the study achieved a partial, but not a full resolution of symptoms. The other open-label study tested verapamil add-on to TAU in women (some pregnant) with BD-I or BD-II. 77\% of the woman (seven out of nine) with mixed states fulfilled response criteria on the mania scale, and two responded on the depression scales as well (Wisner et al. 2002). However, the task force feels that data are still too sparse to make any recommendation either for donepezil nor verapamil.

\section{Electroconvulsive Therapy (ECT)}

\section{Efficacy in acute manic mixed episodes}

In 1938 Cerletti and Bini switched from animal studies to the first ever human trial of ECT in a patient allegedly suffering from a mixed episode with psychotic symptoms (Shorter and Healy 2007). Unfortunately, after this successful start, the role of ECT in the treatment of mixed states diminished over time due to the continued stigma attached to, and lack of access to, this treatment. But evidence from several case series suggest that ECT is effective in the treatment of acute mixed episodes (Valenti et al. 2008). Unfortunately, the original articles do not detail whether patients had ECT as sole therapy or were continued on their medication. 
As it is the usual clinical practice, we assume that all evidence falls into the categories „combination/augmentation therapy”.

In a retrospective study in Aarhus Psychiatric Hospital (Strömgren 1988) a high response to ECT in a sample of 20 patients with ICD-9 manic-depressive mixed states has been observed. Thirteen treatment series were classified as satisfactory response, five as moderate response, one as slightly effective, and one as ineffective. Gruber et al (Gruber et al. 2000) reported a significant reduction in depressive and manic symptoms in a small case series of seven mixed-state patients treated with ECT. Other studies (Devanand et al. 2000) which compared the response to ECT in mixed state, bipolar depression, and mania, showed robust response rates in all groups: $80 \%$ in the mixed group, $100 \%$ in the manic group, and $76 \%$ in the depressed group. Nevertheless, the mixed group required longer hospitalisations and more ECT trials compared to the other two groups. Ciapparelli et al (Ciapparelli et al. 2001) did not find any difference between treatment-resistant bipolar patients with depression $(n=23)$ and mixed manic episodes $(n=41)$ in terms of number of ECT sessions needed but reported a higher response rate of $56 \%$ for mixed mania compared to $26 \%$ for bipolar depression. Overall, he response rates for the bipolar depressed group were slightly lower than those of most other studies. It is possible that the efficacy of ECT was compromised in this study by the concurrent administration of anticonvulsant medications in all patients. Medda et al (Medda et al. 2010) evaluated the ECT response in a sample of BD-I patients, in which 46 patients exhibited depression and 50 patients demonstrated a mixed state. The response rate was similar in bipolar depression and mixed state (67.4\% and $76.0 \%$, respectively), and no difference was found in the remission rate between depression (41.3\%) and mixed state (34.8\%), However, at the end of the ECT course, mixed state may present more residual agitation and psychotic features compared with depressive patients.

The largest case series with mixed patients comes from the Department of Psychiatry of the University of Pisa. Medda et al (Medda et al. 2015) performed an analysis using data obtained from 197 of 203 consecutive patients with a bipolar mixed state, according to DSMIV-TR diagnostic criteria, who were treated with ECT between January 2006 and May 2011. At the end of the ECT course, conducted bilaterally with a brief pulse stimulator twice weekly, 55 patients (27.9\%) were considered non-responders, 82 patients (41.6\%) were responders, and 60 patients (30.5\%) were remitters. Of note, both depressive and manic symptoms were highly responsive to ECT as the significant reductions of respective rating scale scores (YMRS, HAM-D, BPRS and CGI) suggest. ECT was generally well tolerated, in 
six patients only the ECT course was terminated prematurely (one severe confusion, one severe headache, two cardiac arrhythmias, one respiratory complications, and one consent withdrawal). In summary, there are no randomized controlled trials evaluating the efficacy of ECT relative to other treatments in mixed affective states. However, the above reports provide reasonable evidence for the safe and effective use of ECT, including in patients refractory to pharmacotherapy. In addition, response to ECT usually occurred within 3-4 weeks in patients who had previously failed many weeks of pharmacotherapy.

CE in acute manic mixed episodes (monotherapy) is „F”.

$C E$ in acute manic mixed episodes (combination/augmentation therapy) is „C” both for manic and depressive symptoms.

\section{Efficacy in acute depressive mixed episodes}

We could not locate a trial conducted exclusively in bipolar depressive mixed states, but subanalyses of two studies supply some evidence that ECT is effective in what we would term a depressive mixed state. The group from Pisa analysed more in detail which subgroup of mixed patients from the trial by Medda (Medda et al. 2015) had the most profit from ECT (Perugi et al. 2013). Principal component factor analysis (PCA) followed by Varimax rotation was performed on 21 non- overlapping items selected from the Hamilton rating-scale for depression (HAM-D) and from the Young mania rating-scale (YMRS) at baseline evaluation. Cluster subtypes derived from the factor scores were compared in clinical variables and final HAM-D, YMRS, BPRS and CGI scores at baseline and one week after the last ECT session. The principal component factor analysis for the selected HAM-D/YMRS items produced six factors with eigenvalues between 1.6 and 2.6, which explained 55.9\% of the total variance. Cluster analysis identified four groups, including 63 (31.2\%) subjects with Agitated- Irritable Mixed-Depression, 59 (29.2\%) with Psychotic Mixed-Mania, 17 (8.5\%) with AnxiousIrritable- Psychotic Mixed-Mania, and 63 (31.2\%) with Retarded-Psychotic MixedDepression. The Agitated- Irritable Mixed-Depression group had the most profit from ECT in terms of remission rates defined as a CGI severity score $\leq 1$, which were significantly higher than for the other subgroups.

Applying retrospectively mixed states criteria according to the Cincinati criteria (McElroy et al. 1992) and Akiskal's criteria (major depressive episode plus 2-3 manic/hypomanic symptoms) which may be considered as a DSM-5 mixed specifier proxy, Palma et al (Palma et al. 2016) analysed ECT data of a total of 50 ECT course treatments that were performed in 
41 bipolar patients in the Department of Psychiatry at Amadora, Portugal over 5 years. The mixed state group represented 36.6\% $(n=15)$, the depressed group 53.7\% $(n=22)$, and the manic group $9.8 \%(n=4)$ of the total population of patients. All affective episodes, except one mixed state, showed a positive clinical response as documented by retrospective rating of a CGI $\leq 3$. No differences were found in terms of number of ECT sessions performed, length of hospital admission, referral to continuation ECT treatment, number of readmissions, and time until next readmission.

$C E$ in acute depressive mixed episodes (monotherapy) is „F”.

$C E$ in acute depressive mixed episodes (combination/augmentation therapy) is „C”.

\section{Efficacy in maintenance treatment after an acute mixed episode in preventing episodes of any polarity or a new manic, depressive, or mixed episode}

Little is known about the impact of acute ECT on the long-term outcome of bipolar patients.

A first case series reported that maintenance ECT was useful in patients with bipolar disorder initially treated with an course of ECT for an index episode of mania, depression, or mixed state, including rapid cyclers $(\mathrm{N}=4)$ (Vanelle et al. 1994). Medda et al. (Medda et al. 2013) recently published a prospective naturalistic study that followed 36 bipolar patients with a medication-resistant severe depression or mixed state index episode that responded to acute ECT (mean duration $=55.3 \pm$ SD 30.4 weeks). Of these, 13 had a depressive relapse, 5 months on average after the end of acute treatment; one patient had a mixed state relapse. In the study by Palma et al (Palma et al. 2016), eight out of 10 (80.0\%) of the depressive episodes that started cECT were kept on maintenance regime, while the proportion in mixed state group was of 7 out of 11 (63.6\%). The mean duration of follow-up was $136.4 \pm 80.1$ weeks (range: 10.1-249.9 weeks), and no significant differences were observed between depressed and mixed state groups (depressed group $=149.0 \pm$ SD 86.0 versus mixed group $=$ $125.7 \pm$ SD 76.4 weeks, $p=0.344)$. The mixed state group presented a higher percentage of episodes followed by readmission due to affective relapse in comparison to the depressive group. The absolute number of readmissions that followed the index admission, however, did not differ between both groups (average number of readmissions: depressed group $=0.6 \pm \mathrm{SD}$ 1.3 versus mixed group $=0.9 \pm \mathrm{SD} 1.3 ; \mathrm{p}=0.353$ ), neither did the average time to readmission (mixed readmitted patients $=48.3 \pm$ SD 62.0 weeks versus depressed readmitted patients $=47.3 \pm$ SD 48.3 weeks; $p=0.424)$.

CE to prevent a new episode after a mixed index episode (monotherapy): is „F”. CE to prevent a new episode after a mixed index episode (combination/augmentation 
therapy): „C”.

\section{Efficacy in maintenance treatment after an acute manic or depressed episode in preventing new mixed episodes}

We could not locate a study that specifically looked into mixed relapses after a manic or depressive index episode

CE to prevent a mixed episode after a manic or depressed index episode (monotherapy) is „F”.

CE to prevent a mixed episode after a manic or depressed index episode (combination/augmentation therapy) is „, F”.

\section{Further evidence (FE)}

Based on the previously cited case reports and series, we rate the FE: + for short-term treatment, 0 for long- term treatment

\section{Safety \& tolerability (ST)}

Progressive cognitive impairment, especially of memory, is a main worry associated with repetitive ECT sessions. In addition, every session has the inherited risks associated with short-term anaesthesia. Thus, clinicians should take these concerns seriously unless proven otherwise.

Rating of ST: - for short-term treatment, - for long- term treatment

\section{Recommendation grade (RG)}

Given the overall evidence, we would assign a $R G$ „4” both for acute and continuation/maintenance ECT in mixed states. Due to the methodological limitations of ECT research (lack of randomization and placebo control) the evidence is restricted. Nevertheless, ECT may be a useful option in otherwise treatment refractory or frequently relapsing mixed patients.

\section{Other physical treatments}

Some exploratory work has been carried out in the acute treatment of bipolar mixed patients with repetitive transcranial magnetic stimulation (rTMS), Uncontrolled evidence for the use of TMS in mixed patients derives from three case reports, of which at least two report on the same patient. The first one reported the case of a treatment resistant mixed BD-I patient, who 
showed immediate improvement of depressive, but not manic symptoms after treatment with rTMS (Zeeuws et al. 2010a). The same outcome, improvement in depressive but not manic symptoms, was reported in an additional cases of one mixed episode in a patient not responding to ECT (Zeeuws et al. 2010b; Zeeuws et al. 2011).

The use of vagus nerve stimulation (VNS) and Deep Brain Stimulation (DBS) to treat bipolar patients has evolved quite recently, but we could not locate any evidence specifically for mixed patients.

In conclusion, the evidence for physical treatments other than ECT is still too weak to give a recommendation for bipolar mixed patients.

\section{The Role of Psychotherapy and Psychoeducation}

As we clarified at the beginning, this guideline is not focussing on the evidence of psychotherapies and psychoeducation in the treatment of bipolar disorder. The important role of these techniques for improving compliance and resilience against mood instability are well documented and they are an integrative and established component of treatment, accompanying medication. However, the evidence so far is restricted to acute bipolar depression and maintenance treatment, and to our knowledge, no results on psychotherapy in acute mixed patients have been published so far. With regards to prevention of mixed episodes, Colom et al. showed in a randomised controlled trial that adjunctive psychoeducation was effective in delaying the time to a new mixed episode as defined in DSM-IV (Colom et al. 2003).

\section{Conclusion}

Using the established approach of the WFSBP guideline series, and making minor modifications to suit the topic of bipolar mixed states we identified a few medications that appear efficacious in mixed patients. However, none of these medications can fully cover all areas (acute and maintenance treatment) or all subgroups of mixed patient groups equally well, and in summary, the paucity of evidence leaves us with more questions than answers.

Olanzapine, paliperidone and aripiprazole have the best evidence for the treatment of acute mixed manic states. Evidence on a comparative level (RG 1-3) in bipolar depressive mixed 
states only exists for ziprasidone in combination treatment, and on a RG 4 level for carbamazepine, lurasidone, olanzapine and ECT. For the prevention of a new episode after a mixed index episode, quetiapine, lithium and olanzapine appear to be best choice treatments, either in mono-or combination treatment. Less is known how to prevent best a mixed episode after a manic, mixed or depressive index episode, but valproate, olanzapine and lithium have demonstrated at least some effectiveness. In summary, evidence suggests that olanzapine (in mono- or combination treatment) appears as the medication that covers most treatment scenarios and subgroups in mixed patients.

Historically, valproate has been considered an effective treatment in mixed states while lithium was thought to be less effective. In the absence of reliable data for lithium, this may be true for acute treatment, although on low level evidence (RG 4 for valproate). Direct comparison, however, also supports the use of lithium in preventing mixed episodes. Finally, and similar to mania and depression, clozapine and especially ECT are options to consider when other treatments have failed. 


\section{Tables and Figures}

\section{Table 1}

Diagnostic criteria for a mixed episode according to DSM-IV and ICD-10, and for a mixed manic and mixed depressive features specifier according to DSM-5

\begin{tabular}{|c|c|c|c|c|}
\hline & DSM-IV (296.6x) & $\begin{array}{l}\text { ICD-10 (F38 } \\
\text { (single episode) } \\
\text { and F31.6) }\end{array}$ & $\begin{array}{l}\text { DSM-5 mixed } \\
\text { manic features } \\
\text { specifier }\end{array}$ & $\begin{array}{l}\text { DM5 mixed } \\
\text { depressive } \\
\text { features } \\
\text { specifier }\end{array}$ \\
\hline Symptoms & $\begin{array}{l}\text { The symptomatic } \\
\text { criteria are met both } \\
\text { for a Manic Episode } \\
\text { and for a Major } \\
\text { Depressive Episode }\end{array}$ & $\begin{array}{l}\text { The current } \\
\text { episode is } \\
\text { characterized by } \\
\text { either a mixture or } \\
\text { a rapid alternation } \\
\text { (i.e. within a few } \\
\text { hours) of } \\
\text { hypomanic, manic } \\
\text { and depressive } \\
\text { symptoms. }\end{array}$ & $\begin{array}{l}\text { Full criteria are } \\
\text { met for a current } \\
\text { or most-recent } \\
\text { manic episode or } \\
\text { hypomanic } \\
\text { episode and at } \\
\text { least three out of } \\
\text { six specified core } \\
\text { symptoms of } \\
\text { depression are } \\
\text { also present } \\
\text { during the } \\
\text { majority of days } \\
\text { within this } \\
\text { episode. }\end{array}$ & $\begin{array}{l}\text { Full criteria are } \\
\text { met for a major } \\
\text { depressive } \\
\text { episode, and at } \\
\text { least three out } \\
\text { of seven } \\
\text { specific core } \\
\text { manic/ } \\
\text { hypomanic } \\
\text { symptoms are } \\
\text { present during } \\
\text { the majority of } \\
\text { days during the } \\
\text { current or most } \\
\text { recent episode } \\
\text { of depression. }\end{array}$ \\
\hline Time criterion & $\begin{array}{l}\text { Nearly every day } \\
\text { during at least a 1- } \\
\text { week period (time } \\
\text { criterion for a DSM- } \\
\text { IV manic episode) }\end{array}$ & $\begin{array}{l}\text { Both manic and } \\
\text { depressive } \\
\text { symptoms must be } \\
\text { prominent most of } \\
\text { the time during a } \\
\text { period of at least } \\
\text { two weeks. }\end{array}$ & $\begin{array}{l}\text { One week if the } \\
\text { episode is manic, } \\
4 \text { days if the } \\
\text { episode is } \\
\text { hypomanic }\end{array}$ & $\begin{array}{l}\text { Two weeks } \\
\text { (major } \\
\text { depression time } \\
\text { criterion) }\end{array}$ \\
\hline Severity & $\begin{array}{l}\text { The mood } \\
\text { disturbance is } \\
\text { sufficiently severe to }\end{array}$ & No specification & $\begin{array}{l}\text { No specification } \\
\text { except: For } \\
\text { individuals }\end{array}$ & $\begin{array}{l}\text { No } \\
\text { specification } \\
\text { except: For }\end{array}$ \\
\hline
\end{tabular}




\begin{tabular}{|c|c|c|c|c|}
\hline & $\begin{array}{l}\text { cause marked } \\
\text { impairment in } \\
\text { occupational } \\
\text { functioning or in } \\
\text { usual social activities } \\
\text { or relationships with } \\
\text { others, or to } \\
\text { necessitate } \\
\text { hospitalization to } \\
\text { prevent harm to self } \\
\text { or others, or there are } \\
\text { psychotic features. } \\
\text { The fifth digit codes } \\
\text { for severity (0-4) }\end{array}$ & & $\begin{array}{l}\text { whose symptoms } \\
\text { meet full episode } \\
\text { criteria for both } \\
\text { mania and } \\
\text { depression } \\
\text { simultaneously, } \\
\text { the diagnosis } \\
\text { should be manic } \\
\text { episode, with } \\
\text { mixed features, } \\
\text { due to the } \\
\text { marked } \\
\text { impairment and } \\
\text { clinical severity } \\
\text { of full mania. }\end{array}$ & $\begin{array}{l}\text { individuals } \\
\text { whose } \\
\text { symptoms meet } \\
\text { full episode } \\
\text { criteria for both } \\
\text { mania and } \\
\text { depression } \\
\text { simultaneously, } \\
\text { the diagnosis } \\
\text { should be } \\
\text { manic episode, } \\
\text { with mixed } \\
\text { features, }\end{array}$ \\
\hline $\begin{array}{l}\text { Inclusion/ } \\
\text { Exclusion } \\
\text { criteria }\end{array}$ & $\begin{array}{l}\text { The symptoms are } \\
\text { not due to the direct } \\
\text { physiological effects } \\
\text { of a substance (e.g., a } \\
\text { drug of abuse, a } \\
\text { medication, or other } \\
\text { treatment) or a } \\
\text { general medical } \\
\text { condition (e.g., } \\
\text { hyperthyroidism). }\end{array}$ & $\begin{array}{l}\text { The episode is not } \\
\text { attributable to } \\
\text { psychoactive } \\
\text { substance use (F1) } \\
\text { or } \\
\text { any organic mental } \\
\text { disorder, in the } \\
\text { sense of F0. } \\
\text { For F31.6: There } \\
\text { has been at least } \\
\text { one well } \\
\text { authenticated } \\
\text { hypomanic or } \\
\text { manic episode } \\
\text { (F30.-), depressive } \\
\text { (F32.-) or mixed } \\
\text { affective episode } \\
\text { (F38.00) in the } \\
\text { past. }\end{array}$ & $\begin{array}{l}\text { The mixed } \\
\text { symptoms are } \\
\text { not attributable } \\
\text { to the } \\
\text { physiological } \\
\text { effects of a } \\
\text { substance (e.g., a } \\
\text { drug of abuse, } \\
\text { medication, or } \\
\text { other treatment). }\end{array}$ & $\begin{array}{l}\text { The mixed } \\
\text { symptoms are } \\
\text { not attributable } \\
\text { to the } \\
\text { physiological } \\
\text { effects of a } \\
\text { substance (e.g., } \\
\text { a drug of } \\
\text { abuse, } \\
\text { medication, or } \\
\text { other } \\
\text { treatment). }\end{array}$ \\
\hline
\end{tabular}




\section{Table 2}

Categories of Evidence (CE) and recommendation grades (RG)

\begin{tabular}{|c|c|}
\hline $\begin{array}{l}\text { Category of } \\
\text { Evidence }\end{array}$ & Description \\
\hline \multirow[t]{2}{*}{ A } & Full Evidence from Controlled Studies \\
\hline & $\begin{array}{l}\text { is based on: } \\
2 \text { or more double-blind, parallel-group, randomized controlled studies } \\
\text { (RCTs) showing superiority to placebo (or in the case of psychotherapy } \\
\text { studies, superiority to a „psychological placebo“ in a study with adequate } \\
\text { blinding) } \\
\text { and } \\
1 \text { or more positive RCT showing superiority to or equivalent efficacy } \\
\text { compared with established comparator treatment in a three-arm study with } \\
\text { placebo control or in a well-powered non-inferiority trial (only required if } \\
\text { such a standard treatment exists) } \\
\text { In the case of existing negative studies (studies showing non-superiority to } \\
\text { placebo or inferiority to comparator treatment), these must be outweighed by } \\
\text { at least two more positive studies or a meta-analysis of all available studies } \\
\text { showing superiority to placebo and non-inferiority to an established } \\
\text { comparator treatment. } \\
\text { Studies must fulfil established methodological standards. The decision is } \\
\text { based on the primary efficacy measure. }\end{array}$ \\
\hline \multirow[t]{2}{*}{ B } & Limited Positive Evidence from Controlled Studies \\
\hline & $\begin{array}{l}\text { is based on: } \\
1 \text { or more RCTs showing superiority to placebo (or in the case of } \\
\text { psychotherapy studies, superiority to a „psychological placebo“) } \\
\text { or } \\
\text { a randomized controlled comparison with a standard treatment without }\end{array}$ \\
\hline
\end{tabular}




\begin{tabular}{|l|l|}
\hline placebo control with a sample size sufficient for a non-inferiority trial ${ }^{5}$ \\
or \\
a a priori planned, sufficiently powered subgroup analysis as part of the \\
investigational protocol \\
and \\
$\begin{array}{l}\text { In the case of existing negative studies (studies showing non-superiority to } \\
\text { placebo or inferiority to comparator treatment), these must be outweighed by } \\
\text { at least } 1 \text { more positive study or a meta-analysis of all available studies } \\
\text { showing superiority to placebo or at least one more randomized controlled } \\
\text { comparison showing non-inferiority to an established comparator treatment. }\end{array}$ \\
\hline C & $\begin{array}{l}\text { Evidence from Uncontrolled Studies or Case Reports/Expert Opinion/ post } \\
\text { hoc analyses of a RCT }\end{array}$ \\
\hline
\end{tabular}

\footnotetext{
${ }^{5}$ As a result of a consensus process within the task force, we decided to grant a CE "B" also to large size registry studies with real world endpoints and minimized risk of a selection bias, e.g., with each patient serving as his own control. This is the case for one study selected for this review (Joas et al. 2017).
} 


\begin{tabular}{|c|c|}
\hline & $\begin{array}{l}\text { Uncontrolled Studies } \\
\text { is based on: } \\
1 \text { or more positive naturalistic open studies (with a minimum of } 5 \text { evaluable } \\
\text { patients) } \\
\text { or } \\
\text { a comparison with a reference drug with a sample size insufficient for a non- } \\
\text { inferiority trial } \\
\text { and } \\
\text { no negative controlled studies exist } \\
\text { or } \\
\text { Case Reports: } \\
1 \text { or more positive case reports } \\
\text { and } \\
\text { or } \\
\text { no negative controlled studies exist }\end{array}$ \\
\hline $\mathrm{D}$ & $\begin{array}{l}\text { Inconsistent Results } \\
\text { Positive RCTs are outweighed by an approximately equal number of negative } \\
\text { studies }\end{array}$ \\
\hline $\mathrm{E}$ & $\begin{array}{l}\text { Negative Evidence } \\
\text { The majority of RCTs studies or exploratory studies shows non-superiority to } \\
\text { placebo (or in the case of psychotherapy studies, superiority to a }\end{array}$ \\
\hline
\end{tabular}




\begin{tabular}{|l|l|}
\hline & „psychological placebo“) or inferiority to comparator treatment \\
\hline F & Lack of Evidence \\
\hline $\begin{array}{l}\text { Adequate studies proving efficacy or non-efficacy are lacking. } \\
\text { dation } \\
\text { Grades }\end{array}$ & Based on: \\
\hline 1 & Category A evidence and good risk-benefit ratio \\
\hline 2 & Category A evidence and moderate risk-benefit ratio \\
\hline 3 & Category B evidence \\
\hline 4 & Category C evidence \\
\hline
\end{tabular}




\section{Table 3}

Grading of categories FE and ST

\begin{tabular}{|c|c|c|}
\hline & Further evidence (FE) & $\begin{array}{l}\text { Safety \& Tolerability } \\
\text { (ST) }\end{array}$ \\
\hline++ & $\begin{array}{l}\text { Several supportive FE, e.g. meta-analysis or positive studies } \\
\text { which, however, fall short of criteria to be considered as } \\
\text { evidence for CE „A” or „B” for enriched or non-enriched } \\
\text { samples }\end{array}$ & Very good \\
\hline+ & $\begin{array}{l}\text { Some (or more) supportive FE, e.g. limited evidence from } \\
\text { open studies in samples where enrichment is unclear }\end{array}$ & Good \\
\hline 0 & Conflicting data or unknown & $\begin{array}{l}\text { Equally advantages and } \\
\text { disadvantages, or } \\
\text { unknown }\end{array}$ \\
\hline - & $\begin{array}{l}\text { Some (or more) non-supportive FE, e.g., limited negative } \\
\text { evidence from open studies in samples where enrichment is } \\
\text { unclear }\end{array}$ & Some concerns \\
\hline-- & $\begin{array}{l}\text { Several non-supportive FE, e.g. negative meta- analysis or } \\
\text { negative studies which, however, fall short of criteria to be } \\
\text { considered as evidence for CE „A” or „B” for enriched or } \\
\text { non-enriched sample. }\end{array}$ & Major concerns \\
\hline
\end{tabular}




\section{Table 4}

CE and RG for the acute treatment of mixed states

\begin{tabular}{|c|c|c|c|c|c|c|c|}
\hline Agent & $\begin{array}{l}\text { CE in acute MM } \\
\text { episodes } \\
\text { (monotherapy) }\end{array}$ & $\begin{array}{l}\text { CE in acute MM } \\
\text { episodes } \\
\text { (combination/ } \\
\text { augmentation } \\
\text { therapy) }\end{array}$ & $\begin{array}{l}\text { CE in acute DM } \\
\text { episodes } \\
\text { (monotherapy) }\end{array}$ & $\begin{array}{l}\text { CE in acute DM } \\
\text { episodes } \\
\text { (combination/ } \\
\text { augmentation } \\
\text { therapy) }\end{array}$ & $\begin{array}{l}\text { FE in short } \\
\text { term } \\
\text { treatment }\end{array}$ & $\begin{array}{l}\text { ST in short } \\
\text { term } \\
\text { treatment }\end{array}$ & $\begin{array}{l}\text { RG for the acute } \\
\text { treatment of } \\
\text { mixed states }\end{array}$ \\
\hline Antidepressants & $\mathrm{F}$ & $\mathrm{F}$ & $\mathrm{F}$ & $\mathrm{F}$ & 0 & - & none \\
\hline Asenapine & $\begin{array}{l}\text { E for MS } \\
\text { C for DS }\end{array}$ & $\mathrm{F}$ & $\mathrm{F}$ & $\mathrm{F}$ & + & + & $\begin{array}{l}4 \text { for MM } \\
\text { (monotherapy, for } \\
\text { DS only) }\end{array}$ \\
\hline Cariprazine & C for MS & $\mathrm{F}$ & $\mathrm{F}$ & $\mathrm{F}$ & 0 & + & 4 for $M M$ \\
\hline
\end{tabular}




\begin{tabular}{|c|c|c|c|c|c|c|c|}
\hline & F for DS & & & & & & (monotherapy, for \\
\hline Clozapine & C for MS & C for MS & $\mathrm{F}$ & $\mathrm{F}$ & + & - & $\begin{array}{l}\text { for MM } \\
\text { (monotherapy and } \\
\text { combination } \\
\text { therapy, MS only) }\end{array}$ \\
\hline Lamotrigine & $\mathrm{F}$ & $\mathrm{F}$ & $\mathrm{F}$ & $\mathrm{F}$ & + & + & none \\
\hline Olanzapine & $\begin{array}{l}\text { A for MS } \\
\text { C for DS }\end{array}$ & $\begin{array}{l}\text { B for MS } \\
\text { B for DS }\end{array}$ & $\mathrm{C}$ & $\bar{F}$ & ++ & + & $\begin{array}{l}2 \text { for MM } \\
\text { (monotherapy) } \\
3 \text { for MM } \\
\text { (combination } \\
\text { therapy with } \\
\text { valproate) } \\
4 \text { for DM }\end{array}$ \\
\hline
\end{tabular}




\begin{tabular}{|c|c|c|c|c|c|c|c|}
\hline & & & & & & & (monotherapy) \\
\hline Paliperidone & $\begin{array}{l}\text { B for MS } \\
\text { E for DS }\end{array}$ & $\mathrm{E}$ & $\mathrm{F}$ & $\mathrm{F}$ & 0 & 0 & $\begin{array}{l}3 \text { for MM } \\
\text { (monotherapy) }\end{array}$ \\
\hline Quetiapine & $\mathrm{E}$ & $\begin{array}{l}\text { C for MS } \\
\text { B for DS }\end{array}$ & $\mathrm{F}$ & $\mathrm{F}$ & + & 0 & $\begin{array}{l}3 \text { for MM } \\
\text { (combination, for } \\
\text { DS) } \\
4 \text { for MM } \\
\text { (combination, for } \\
\text { MS) }\end{array}$ \\
\hline Risperidone & $\mathrm{C}$ & $\mathrm{E}$ & $\mathrm{F}$ & $\mathrm{F}$ & + & 0 & $\begin{array}{l}4 \text { for MM } \\
\text { (monotherapy) }\end{array}$ \\
\hline Topiramate & $\mathrm{F}$ & D for MS & F & $\mathrm{F}$ & + & 0 & $\begin{array}{l}5 \text { for MM } \\
\text { (combination } \\
\text { therapy) }\end{array}$ \\
\hline Typical AP & $\mathrm{C}$ & $\mathrm{E}$ & F & $\mathrm{F}$ & 0 & - & 4 for $\mathrm{MM}$ \\
\hline
\end{tabular}




\begin{tabular}{|c|c|c|c|c|c|c|c|}
\hline & & & & & & & (monotherapy) \\
\hline Ziprasidone & $\begin{array}{l}\text { C for MS } \\
\text { C for DS }\end{array}$ & $\mathrm{F}$ & $\mathrm{F}$ & B & + & + & $\begin{array}{l}4 \text { for MM } \\
\text { (monotherapy) } \\
3 \text { for DM } \\
\text { (combination) }\end{array}$ \\
\hline ECT & $\mathrm{F}$ & $\mathrm{C}$ & $\mathrm{F}$ & $\mathrm{C}$ & 0 & - & $\begin{array}{l}4 \text { for MM } \\
\text { (combination) } \\
4 \text { for DM } \\
\text { (combination) }\end{array}$ \\
\hline
\end{tabular}

MM: manic mixed; DM: depressive mixed, MS: Manic symptoms; DS: Depressive symptoms; M: Mania, D: Depression, Mx: Mixed episode, MIE: Manic index episode, DIE: Depressive index episode, MxIE: Mixed index episode, CE: Category of Evidence, FE: Further evidence, ST: Safety \& Tolerability, RG : Recommendation grade

\section{Table 5}

CE and RG for the maintenance treatment after a mixed episode and for the prevention of mixed episodes 


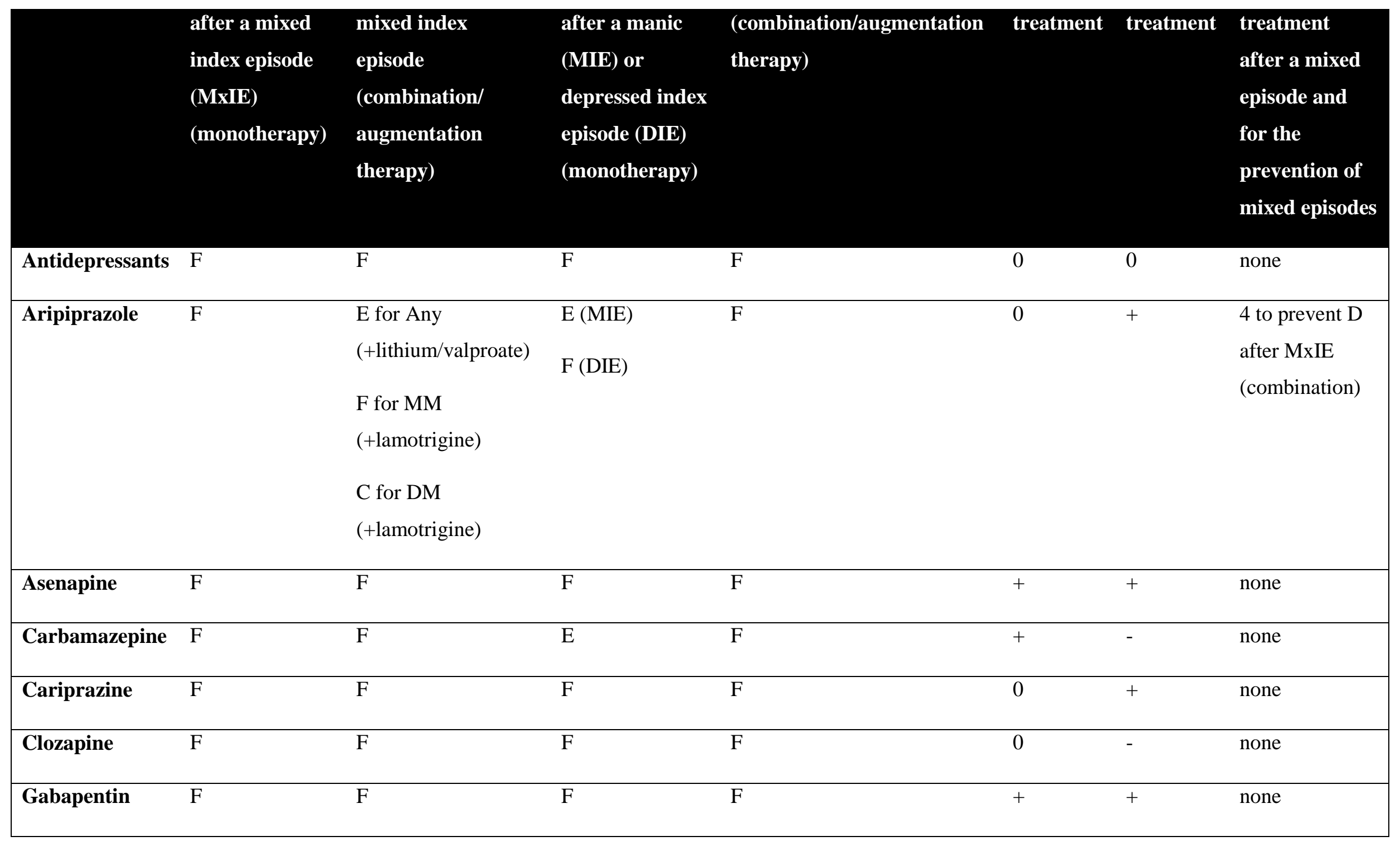




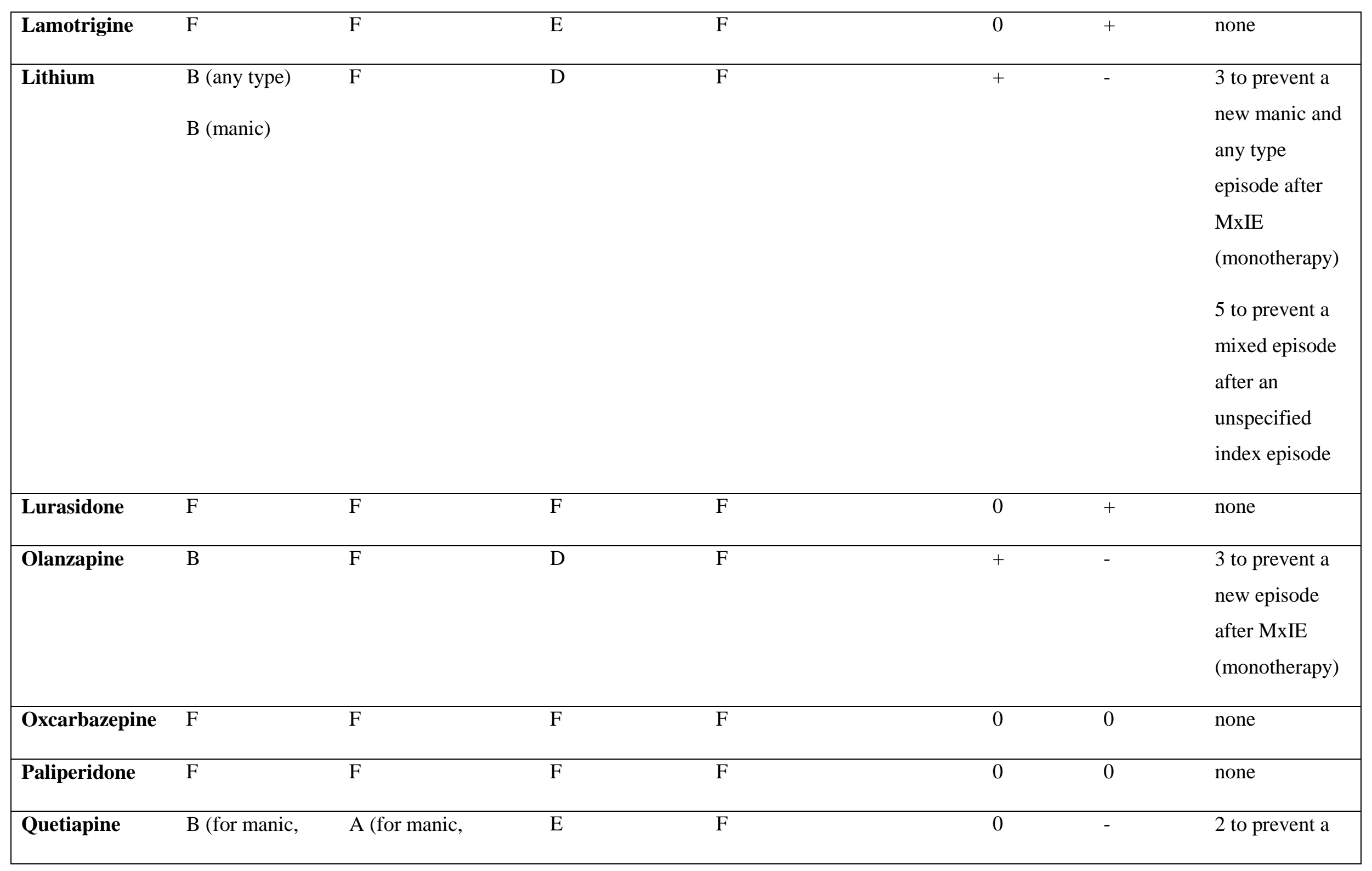




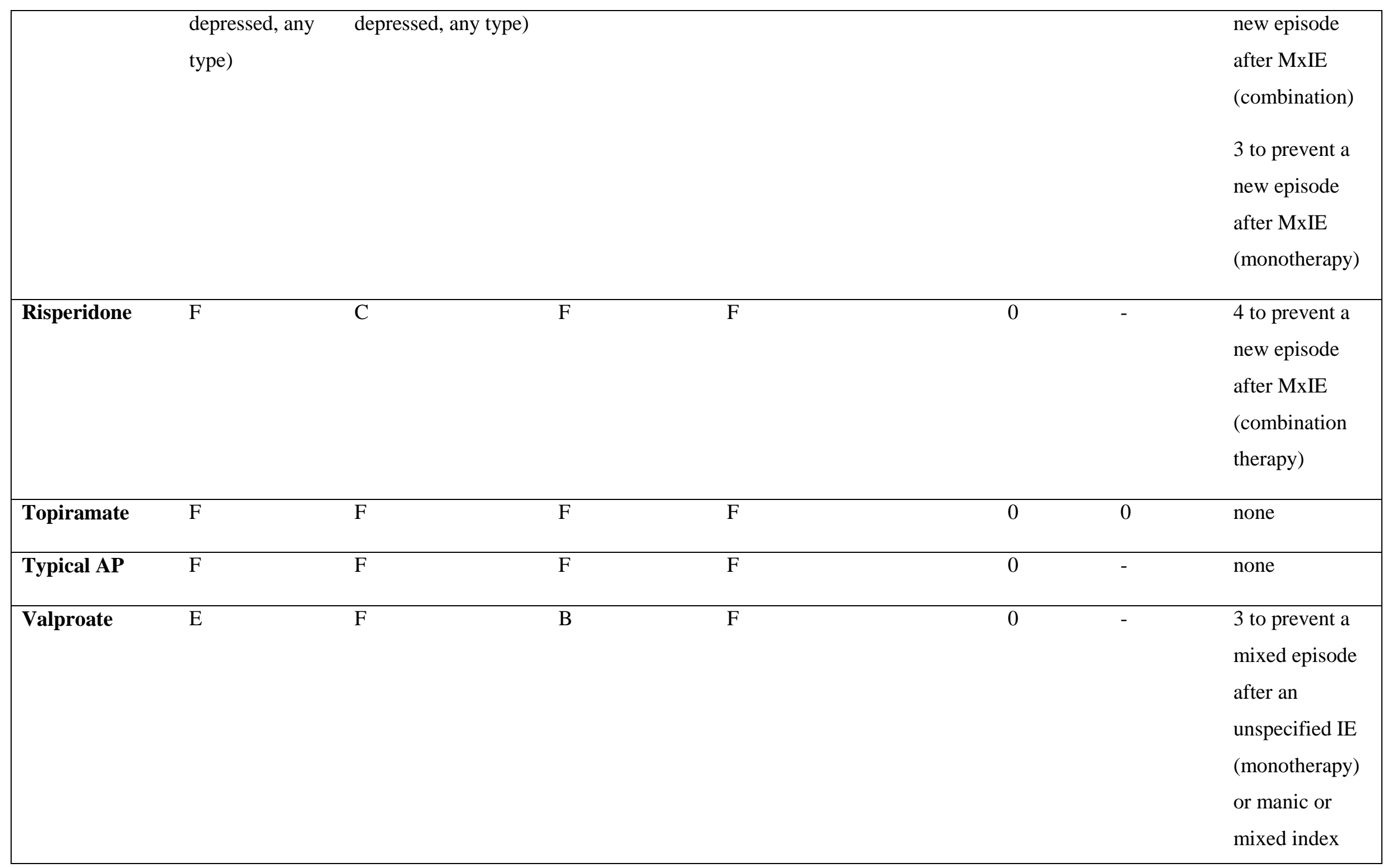




\begin{tabular}{|c|c|c|c|c|c|c|c|}
\hline & & & & & & & episode \\
\hline
\end{tabular}

MM: manic mixed; DM: depressive mixed, M: Mania, D: Depression, Mx: Mixed episode, MIE: Manic index episode, DIE: Depressive index episode, MxIE: Mixed index episode, CE: Category of Evidence, FE: Further evidence, ST: Safety \& Tolerability, RG: Recommendation grade

\section{Figure 1}

Evidence based treatments for mixed episodes, in mono- or combination treatment. For details (e.g., whether a medication is effective only against manic or depressive symptoms, or any symptoms) we refer to the respective chapters and Tables $\underline{4}$ and $\underline{5}$ (MS = mood stabilizer (lithium or valproate), TAU = Treatment as usual) 


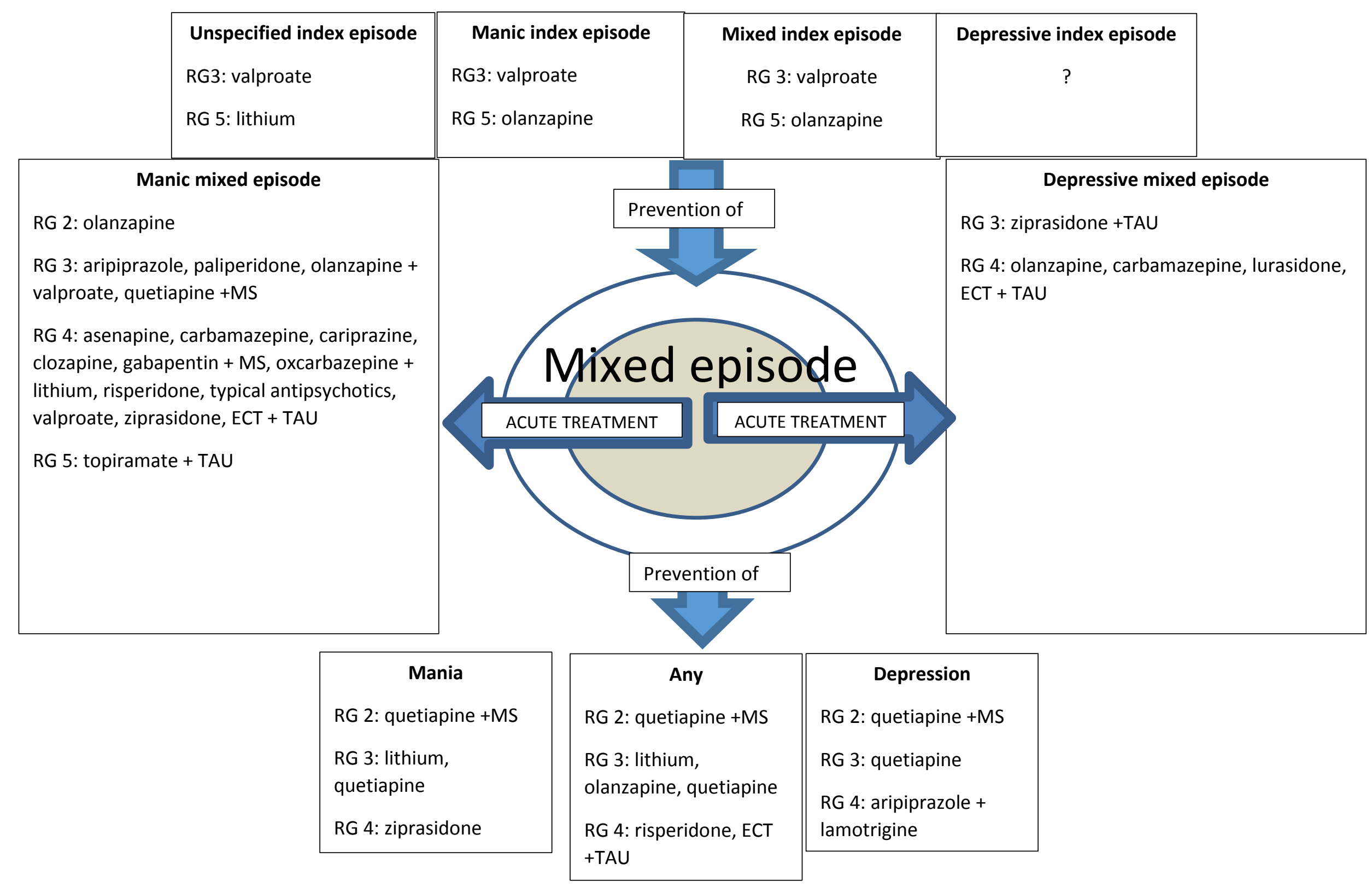




\section{References}

Akiskal HS (1992) The distinctive mixed states of bipolar I, II, and III. Clin Neuropharmacol 15 Suppl 1:632-633.

Akiskal HS (1994) Dysthymic and cyclothymic depressions: therapeutic considerations. J Clin Psychiatry 55 Suppl:46-52.:46-52.

Akiskal HS, Benazzi F (2004) Validating Kraepelin's two types of depressive mixed states: "depression with flight of ideas" and "excited depression". World J Biol Psychiatry 5:107113.

Akiskal HS, Hantouche EG, Bourgeois ML, Azorin J-M, Sechter D, Allilaire J-F, Lancrenon S, Fraud J-P, Châtenet-Duchêne L (1998) Gender, temperament, and the clinical picture in dysphoric mixed mania: Findings from a French national study (EPIMAN). J Affect Disord 50:175-186.

Altshuler LL, Keck PE, Jr., McElroy SL, Suppes T, Brown ES, Denicoff K, Frye M, Gitlin M, Hwang S, Goodman R, et al. (1999) Gabapentin in the acute treatment of refractory bipolar disorder. Bipolar Disord 1:61-65.

American Psychiatric Association. 2013. Diagnostic and statistical manual of mental disorders. 5th ed. APA Press, Washington, DC.

American Psychiatric Association. 1987. Diagnostic and statistical manual of mental disorders. 3rd revised ed. APA Press, Washington DC.

American Psychiatric Association. 1994. Diagnostic and statistical manual of mental disorders. 4th revised ed. APA Press, Washington DC.

Arnold LM, McElroy SL, Keck PE (2000) The role of gender in mixed mania. Compr Psychiatry 41:83-87.

Azorin JM, Aubrun E, Bertsch J, Reed C, Gerard S, Lukasiewicz M (2009) Mixed states vs. pure mania in the French sample of the EMBLEM study: results at baseline and 24 months--European mania in bipolar longitudinal evaluation of medication. BMC Psychiatry 9:33.

Azorin JM, Sapin C, Weiller E (2013) Effect of asenapine on manic and depressive symptoms in bipolar I patients with mixed episodes: results from post hoc analyses. J Affect Disord 145:62-69.

Backlund L, Ehnvall A, Hetta J, Isacsson G, Agren H (2009) Identifying predictors for good lithium response - a retrospective analysis of 100 patients with bipolar disorder using a life-charting method. Eur Psychiatry 24:171-177.

Baillarger J (1854) Folie à double forme.Masson, Paris.

Baker RW, Brown E, Akiskal HS, Calabrese JR, Ketter TA, Schuh LM, Trzepacz PT, Watkin JG, Tohen M (2004) Efficacy of olanzapine combined with valproate or lithium in the treatment of dysphoric mania. Br J Psychiatry 185:472-478. 
Baker RW, Tohen M, Fawcett J, Risser RC, Schuh LM, Brown E, Stauffer VL, Shao L, Tollefson GD (2003) Acute dysphoric mania: treatment response to olanzapine versus placebo. J Clin Psychopharmacol 23:132-137.

Balazs J, Benazzi F, Rihmer Z, Rihmer A, Akiskal KK, Akiskal HS (2006) The close link between suicide attempts and mixed (bipolar) depression: implications for suicide prevention. J Affect Disord 91:133-138.

Baldessarini RJ, Hennen J, Wilson M, Calabrese J, Chengappa R, Keck PE, Jr., McElroy SL, Sachs G, Vieta E, Welge JA, et al. (2003) Olanzapine versus placebo in acute mania: treatment responses in subgroups. J Clin Psychopharmacol 23:370-376.

Baldessarini RJ, Tondo L, Visioli C (2014) First-episode types in bipolar disorder: predictive associations with later illness. Acta Psychiatr Scand 129:383-392.

Bandelow B, Zohar J, Hollander E, Kasper S, Möller HJ, on behalf of the WFSBP Task Force on Treatment Guidelines for Anxiety Obsessive-Compulsive Post-traumatic Stress Disorders (2008a) World Federation of Societies of Biological Psychiatry (WFSBP) guidelines for the pharmacological treatment of anxiety, obsessive-compulsive and posttraumatic stress disorders - first revision. World J Biol Psychiatry 9:248-312.

Bandelow B, Zohar J, Kasper S, Moller HJ (2008b) How to grade categories of evidence. World J Biol Psychiatry 9:242-247.

Barzman DH, DelBello MP, Kowatch RA, Warner J, Rofey D, Stanford K, Rappaport K, Daniels JP, Strakowski SM (2005) Adjunctive topiramate in hospitalized children and adolescents with bipolar disorders. J Child Adolesc Psychopharmacol 15:931-937.

Benabarre A, Vieta E, Colom F, Martinez A, Reinares M, Corbella B (2001) Treatment of mixed mania with risperidone and mood stabilizers. Can J Psychiatry 46:866-867.

Benazzi F (2005) Mixed depression: a clinical marker of bipolar-II disorder. Prog Neuropsychopharmacol Biol Psychiatry 29:267-274.

Benazzi F (2006) Mixed depression, suicidality, and antidepressants. J Clin Psychiatry 67:1650-1651.

Benazzi F, Berk M, Frye MA, Wang W, Barraco A, Tohen M (2009) Olanzapine/fluoxetine combination for the treatment of mixed depression in bipolar I disorder: a post hoc analysis. J Clin Psychiatry 70:1424-1431.

Benazzi F, Koukopoulos A, Akiskal HS (2004) Toward a validation of a new definition of agitated depression as a bipolar mixed state (mixed depression). Eur Psychiatry 19:85-90.

Benedetti A, Lattanzi L, Pini S, Musetti L, Dell'Osso L, Cassano GB (2004) Oxcarbazepine as add-on treatment in patients with bipolar manic, mixed or depressive episode. J Affect Disord 79:273-277.

Berk M, Tiller JW, Zhao J, Yatham LN, Malhi GS, Weiller E (2015) Effects of asenapine in bipolar I patients meeting proxy criteria for moderate-to-severe mixed major depressive episodes: a post hoc analysis. J Clin Psychiatry 76:728-734. 
Berwaerts J, Lane R, Nuamah IF, Lim P, Remmerie B, Hough DW (2011) Paliperidone extended-release as adjunctive therapy to lithium or valproate in the treatment of acute mania: a randomized, placebo-controlled study. J Affect Disord 129:252-260.

Berwaerts J, Melkote R, Nuamah I, Lim P (2012a) A randomized, placebo- and activecontrolled study of paliperidone extended-release as maintenance treatment in patients with bipolar I disorder after an acute manic or mixed episode. J Affect Disord 138:247-258.

Berwaerts J, Xu H, Nuamah I, Lim P, Hough D (2012b) Evaluation of the efficacy and safety of paliperidone extended-release in the treatment of acute mania: a randomized, doubleblind, dose-response study. J Affect Disord 136:e51-e60.

Birmaher B, Axelson D, Strober M, Gill MK, Valeri S, Chiappetta L, Ryan N, Leonard H, Hunt J, Iyengar S, et al. (2006) Clinical course of children and adolescents with bipolar spectrum disorders. Arch Gen Psychiatry 63:175-183.

Bottlender R, Sato T, Kleindienst N, Strauß A, Möller H-J (2002) Mixed depressive features predict maniform switch during treatment of depression in bipolar I disorder. $\mathrm{J}$ Affect.Disord., in press

Bowden CL, Brugger AM, Swann AC, Calabrese JR, Janicak PG, Petty F, Dilsaver SC, Davis JM, Rush AJ, Small JG, et al. (1994) Efficacy of divalproex vs lithium and placebo in the treatment of mania. The Depakote Mania Study Group. JAMA 271:918-924.

Bowden CL, Calabrese JR, McElroy SL, Gyulai L, Wassef A, Petty F, Pope HG, Chou JC, Keck PE, Rhodes LJ, et al. (2000) A randomized, placebo-controlled 12-month trial of divalproex and lithium in treatment of outpatients with bipolar I disorder. Divalproex Maintenance Study Group. Arch Gen Psychiatry 57:481-489.

Bowden CL, Calabrese JR, Sachs G, Yatham LN, Asghar SA, Hompland M, Montgomery P, Earl N, Smoot TM, DeVeaugh-Geiss J (2003) A placebo-controlled 18-month trial of lamotrigine and lithium maintenance treatment in recently manic or hypomanic patients with bipolar I disorder. Arch Gen Psychiatry 60:392-400.

Bowden CL, Collins MA, McElroy SL, Calabrese JR, Swann AC, Weisler RH, Wozniak PJ (2005) Relationship of mania symptomatology to maintenance treatment response with divalproex, lithium, or placebo. Neuropsychopharmacology 30:1932-1939.

Bowden CL, Swann AC, Calabrese JR, Rubenfaer LM, Wozniak PJ, Collins MA, bi-Saab W, Saltarelli M (2006) A randomized, placebo-controlled, multicenter study of divalproex sodium extended release in the treatment of acute mania. J Clin Psychiatry 67:1501-1510.

Bowden CL, Vieta E, Ice KS, Schwartz JH, Wang PP, Versavel M (2010) Ziprasidone plus a mood stabilizer in subjects with bipolar I disorder: a 6-month, randomized, placebocontrolled, double-blind trial. J Clin Psychiatry 71:130-137.

Brieger P, Roettig S, Ehrt U, Wenzel A, Bloink R, Marneros A (2003) TEMPS-a scale in 'mixed' and 'pure' manic episodes: new data and methodological considerations on the relevance of joint anxious-depressive temperament traits. J Affect Disord 73:99-104.

Brown ES, Davila D, Nakamura A, Carmody TJ, Rush AJ, Lo A, Holmes T, Adinoff B, Caetano R, Swann AC, et al. (2014) A randomized, double-blind, placebo-controlled trial 
of quetiapine in patients with bipolar disorder, mixed or depressed phase, and alcohol dependence. Alcohol Clin Exp Res 38:2113-2118.

Burt T, Sachs GS, Demopulos C (1999) Donepezil in treatment-resistant bipolar disorder. Biol Psychiatry 45:959-964.

Calabrese J, Bowden CL, Sachs G, Yatham LN, Behnke K, Mehtonen O-P, Montgomery P, Ascher J, Paska W, Earl NL, et al. (2003) A Placebo-Controlled 18-Month Trial of Lamotrigine and Lithium Maintenance Treatment in Recently Depressed Patients with Bipolar I Disorder. J Clin Psychiatry 64:1024.

Calabrese JR, Keck PE, Jr., Starace A, Lu K, Ruth A, Laszlovszky I, Nemeth G, Durgam S (2015) Efficacy and safety of low- and high-dose cariprazine in acute and mixed mania associated with bipolar I disorder: a double-blind, placebo-controlled study. J Clin Psychiatry 76:284-292.

Calabrese JR, Sanchez R, Jin N, Amatniek J, Cox K, Johnson B, Perry P, Hertel P, Such P, Salzman PM, et al. (2017) Efficacy and Safety of Aripiprazole Once-Monthly in the Maintenance Treatment of Bipolar I Disorder: A Double-Blind, Placebo-Controlled, 52Week Randomized Withdrawal Study. J Clin Psychiatry 78:324-331.

Calabrese JR, Shelton MD, Rapport DJ, Youngstrom EA, Jackson K, Bilali S, Ganocy SJ, Findling RL (2005) A 20-month, double-blind, maintenance trial of lithium versus divalproex in rapid-cycling bipolar disorder. Am J Psychiatry 162:2152-2161.

Camm AJ, Karayal ON, Meltzer H, Kolluri S, O'Gorman C, Miceli J, Tensfeldt T, Kane JM (2012) Ziprasidone and the corrected QT interval: a comprehensive summary of clinical data. CNS Drugs 26:351-365.

Caresano C, Di SG, Fagiolini A, Maina G, Perugi G, Ripellino C, Vampini C (2014) Costeffectiveness of asenapine in the treatment of patients with bipolar I disorder with mixed episodes in an Italian context. Adv Ther 31:873-890.

Carlson BX, Ketter TA, Sun W, Timko K, McQuade RD, Sanchez R, Vester-Blokland E, Marcus R (2012) Aripiprazole in combination with lamotrigine for the long-term treatment of patients with bipolar I disorder (manic or mixed): a randomized, multicenter, doubleblind study (CN138-392). Bipolar Disord 14:41-53.

Carroll BJ (1983) Neurobiological dimensions of mania and depression. In: Angst J (ed) The Origins of Depression: Current Concepts and Approaches.Springer, New York: pp 163186.

Cassidy F, Ahearn E, Carroll BJ (2001a) A prospective study of inter-episode consistency of manic and mixed subtypes of bipolar disorder. J Affect Disord 67:181-185.

Cassidy F, Ahearn EP, Carroll BJ (2001b) Substance abuse in bipolar disorder. Bipolar Disord 3:181-188.

Cassidy F, Carroll BJ (2001) The clinical epidemiology of pure and mixed manic episodes. Bipolar Disord 3:35-40.

Cassidy F, Murry E, Forest K, Carroll BJ (1998) Signs and symptoms of mania in pure and mixed episodes. J Affect Disord 50:187-201. 
Cassidy F, Yatham LN, Berk M, Grof P (2008) Pure and mixed manic subtypes: a review of diagnostic classification and validation. Bipolar Disord 10:131-143.

Catapano-Friedman L (2001) Effectiveness of quetiapine in the management of psychotic depression in an adolescent boy with bipolar disorder, mixed, with psychosis. J Child Adolesc Psychopharmacol 11:205-206.

Chengappa KN, Rathore D, Levine J, Atzert R, Solai L, parepally H, Levin H, moffa N, Delaney J, Brar JS (1999) Topiramate as add-on treatment for patients with bipolar mania. Bipolar disorders 1:42-53.

Ciapparelli A, Dell'Osso L, Tundo A, Pini S, Chiavacci MC, Di S, I, Cassano GB (2001) Electroconvulsive therapy in medication-nonresponsive patients with mixed mania and bipolar depression. J Clin Psychiatry 62:552-555.

Cipriani A, Reid K, Young AH, Macritchie K, Geddes J (2013) Valproic acid, valproate and divalproex in the maintenance treatment of bipolar disorder. Cochrane Database Syst RevCD003196.

Citrome L (2014) Asenapine review, part II: clinical efficacy, safety and tolerability. Expert Opin Drug Saf 13:803-830.

Citrome L (2013) Cariprazine in bipolar disorder: clinical efficacy, tolerability, and place in therapy. Adv Ther 30:102-113.

Citrome L (2011) Drug safety evaluation of ziprasidone. Expert Opin Drug Saf 10:437-448.

Clothier J, Swann AC, Freeman T (1992) Dysphoric mania. J Clin Psychopharmacol 12:13S$16 \mathrm{~S}$.

Colom F, Vieta E, Martinez-Aran A, Reinares M, Goikolea JM, Benabarre A, Torrent C, Comes M, Corbella B, Parramon G, et al. (2003) A randomized trial on the efficacy of group psychoeducation in the prophylaxis of recurrences in bipolar patients whose disease is in remission. Arch Gen Psychiatry 60:402-407.

Craney JL, Geller B (2003) A prepubertal and early adolescent bipolar disorder-I phenotype: review of phenomenology and longitudinal course. Bipolar Disord 5:243-256.

Cutler AJ, Datto C, Nordenhem A, Minkwitz M, Acevedo L, Darko D (2011) Extendedrelease quetiapine as monotherapy for the treatment of adults with acute mania: a randomized, double-blind, 3-week trial. Clin Ther 33:1643-1658.

Dannlowski U, Baune BT, Bockermann I, Domschke K, Evers S, Arolt V, Hetzel G, Rothermundt M (2008) Adjunctive antidepressant treatment with quetiapine in agitated depression: positive effects on symptom reduction, psychopathology and remission rates. Hum psychopharmacol 23:587-593.

Dell'Osso L, Placidi GF, Nassi R, Freer P, Cassano GB, Akiskal HS (1991) The manicdepressive mixed state: familial, temperamental and psychopathologic characteristics in 108 female inpatients. Eur Arch Psychiatry Clin Neurosci 240:234-239.

Devanand DP, Polanco P, Cruz R, Shah S, Paykina N, Singh K, Majors L (2000) The efficacy of ECT in mixed affective states. J ECT 16:32-37. 
Diav-Citrin O, Shechtman S, Ornoy S, Arnon J, Schaefer C, Garbis H, Clementi M, Ornoy A (2005) Safety of haloperidol and penfluridol in pregnancy: a multicenter, prospective, controlled study. J Clin Psychiatry 66:317-322.

Dilsaver SC, Chen YW, Swann AC, Shoaib AM, Krajewski KJ (1994) Suicidality in patients with pure and depressive mania. Am J Psychiatry 151:1312-1315.

Dilsaver SC, Swann AC (1995) Mixed mania: apparent induction by a tricyclic antidepressant in five consecutively treated patients with bipolar depression. Biol Psychiatry 37:60-62.

Durgam S, Earley W, Lipschitz A, Guo H, Laszlovszky I, Nemeth G, Vieta E, Calabrese JR, Yatham LN (2016) An 8-Week Randomized, Double-Blind, Placebo-Controlled Evaluation of the Safety and Efficacy of Cariprazine in Patients With Bipolar I Depression. Am J Psychiatry 173:271-281.

Durgam S, Starace A, Li D, Migliore R, Ruth A, Nemeth G, Laszlovszky I (2015) The efficacy and tolerability of cariprazine in acute mania associated with bipolar I disorder: a phase II trial. Bipolar Disord 17:63-75.

Earley W, Durgam S, Lu K, Debelle M, Laszlovszky I, Vieta E, Yatham LN (2017) Tolerability of cariprazine in the treatment of acute bipolar I mania: A pooled post hoc analysis of 3 phase II/III studies. J Affect Disord 215:205-212.

El-Mallakh RS, Ghaemi SN, Sagduyu K, Thase ME, Wisniewski SR, Nierenberg AA, Zhang HW, Pardo TA, Sachs G (2008) Antidepressant-associated chronic irritable dysphoria (ACID) in STEP-BD patients. J Affect Disord 111:372-377.

El-Mallakh RS, Vieta E, Rollin L, Marcus R, Carson WH, McQuade R (2010) A comparison of two fixed doses of aripiprazole with placebo in acutely relapsed, hospitalized patients with bipolar disorder I (manic or mixed) in subpopulations (CN138-007). Eur Neuropsychopharmacol 20:776-783.

Ernst CL, Goldberg JF (2002) The reproductive safety profile of mood stabilizers, atypical antipsychotics, and broad-spectrum psychotropics. J Clin Psychiatry 63 Suppl 4:42-55.

European Medicines Agency scientific commitee CfMPfHU(14-8-2016) Concept paper on the need for revision of the note for guidance on clinical investigation of medicinal products for the treatment and prevention of bipolar disorder. Electronic Citation http://www.ema.europa.eu/docs/en_GB/document_library/Scientific_guideline/2016/07/W C500211317.pdf

Findling RL, Ginsberg LD (2014) The safety and effectiveness of open-label extended-release carbamazepine in the treatment of children and adolescents with bipolar I disorder suffering from a manic or mixed episode. Neuropsychiatr Dis Treat 10:1589-1597.

Flint J, Cuijpers P, Horder J, Koole SL, Munafo MR (2015) Is there an excess of significant findings in published studies of psychotherapy for depression? Psychol Med 45:439-446.

Fornaro M, Stubbs B, De BD, Perna G, Valchera A, Veronese N, Solmi M, Gananca L (2016) Atypical Antipsychotics in the Treatment of Acute Bipolar Depression with Mixed Features: A Systematic Review and Exploratory Meta-Analysis of Placebo-Controlled Clinical Trials. Int J Mol Sci 17:241. 
Frazier JA, Meyer MC, Biederman J, Wozniak J, Wilens TE, Spencer TJ, Kim GS, Shapiro S (1999) Risperidone treatment for juvenile bipolar disorder: a retrospective chart review. J Am Acad Child Adolesc Psychiatry 38:960-965.

Freeman MP, Freeman SA, McElroy SL (2002) The comorbidity of bipolar and anxiety disorders: prevalence, psychobiology, and treatment issues. J Affect Disord 68:1-23.

Freeman TW, Clothier JL, Pazzaglia P, Lesem MD, Swann AC (1992) A double-blind comparison of valproate and lithium in the treatment of acute mania. Am J Psychiatry 149:108-111.

Garcia-Bonetto GM, Nieto IR, Chapa R, Adrianzen C, Brnabic A, Meyers AL, Granger RE, Dossenbach M, Karagianis J, Ruiz I (2009) Pharmacological treatment outcomes in Latin American patients with bipolar I disorder. Archivos de Neurosciencias 14:215-223.

Garriga M, Pacchiarotti I, Kasper S, Zeller SL, Allen MH, Vazquez G, Baldacara L, San L, lister-Williams RH, Fountoulakis KN, et al. (2016) Assessment and management of agitation in psychiatry: Expert consensus. World J Biol Psychiatry 17:86-128.

Geller B, Tillman R, Craney JL, Bolhofner K (2004) Four-year prospective outcome and natural history of mania in children with a prepubertal and early adolescent bipolar disorder phenotype. Arch Gen Psychiatry 61:459-467.

Ghaemi SN (2008) All mixed up: on the absence of diagnostic guidelines for mixed states in the ISBD Diagnostic Guidelines Task Force Report. Bipolar Disord 10:129-130.

Goldberg JF, Perlis RH, Bowden CL, Thase ME, Miklowitz DJ, Marangell LB, Calabrese JR, Nierenberg AA, Sachs GS (2009) Manic symptoms during depressive episodes in 1,380 patients with bipolar disorder: findings from the STEP-BD. Am J Psychiatry 166:173-181.

Gonzalez-Pinto A, Aldama A, Mosquera F, Gonzalez GC (2007) Epidemiology, diagnosis and management of mixed mania. CNS Drugs 21:611-626.

Gonzalez-Pinto A, Barbeito S, Alonso M, Alberich S, Haidar MK, Vieta E, TabaresSeisdedos R, Zorrilla I, Gonzalez-Pinto MA, Lopez P (2011) Poor long-term prognosis in mixed bipolar patients: 10-year outcomes in the Vitoria prospective naturalistic study in Spain. J Clin Psychiatry 72:671-676.

Gonzalez-Pinto A, Tohen M, Lalaguna B, Perez-Heredia JL, Fernandez-Corres B, Gutierrez M, Mico JA (2002) Treatment of bipolar I rapid cycling patients during dysphoric mania with olanzapine. J Clin Psychopharmacol 22:450-454.

Goodwin GM, Haddad PM, Ferrier IN, Aronson JK, Barnes T, Cipriani A, Coghill DR, Fazel S, Geddes JR, Grunze H, et al. (2016) Evidence-based guidelines for treating bipolar disorder: Revised third edition recommendations from the British Association for Psychopharmacology. J Psychopharmacol 30:495-553.

Grande I, Berk M, Birmaher B, Vieta E (2016) Bipolar disorder. Lancet 387:1561-1572.

Grande I, Hidalgo-Mazzei D, Nieto E, Mur M, Saez C, Forcada I, Vieta E (2015) Asenapine prescribing patterns in the treatment of manic in- and outpatients: Results from the MANACOR study. Eur Psychiatry 30:528-534. 
Gruber NP, Dilsaver SC, Shoaib AM, Swann AC (2000) ECT in mixed affective states: a case series. J ECT 16:183-188.

Grunze H, Azorin JM (2014) Clinical decision making in the treatment of mixed states. World J Biol Psychiatry 15:355-368.

Grunze H, Erfurth A, Amann B, Giupponi G, Kammerer C, Walden J (1999) Intravenous valproate loading in acutely manic and depressed bipolar I patients. J Clin Psychopharmacol 19:303-309.

Grunze H, Vieta E, Goodwin GM, Bowden C, Licht RW, Moller HJ, Kasper S (2013) The World Federation of Societies of Biological Psychiatry (WFSBP) Guidelines for the Biological Treatment of Bipolar Disorders: Update 2012 on the long-term treatment of bipolar disorder. World J Biol Psychiatry 14:154-219.

Grunze H, Vieta E, Goodwin GM, Bowden C, Licht RW, Moller HJ, Kasper S (2009) The World Federation of Societies of Biological Psychiatry (WFSBP) Guidelines for the Biological Treatment of Bipolar Disorders: Update 2009 on the Treatment of Acute Mania. World J Biol Psychiatry 10:85-116.

Grunze H, Vieta E, Goodwin GM, Bowden C, Licht RW, Moller HJ, Kasper S (2010) The World Federation of Societies of Biological Psychiatry (WFSBP) Guidelines for the Biological Treatment of Bipolar Disorders: Update 2010 on the treatment of acute bipolar depression. World J Biol Psychiatry 11:81-109.

Grunze H, Walden J (2005) Biological aspects of rapid cycling and mixed states. In: Marneros A, Goodwin F (eds) Bipolar Disorders. Mixed States, Rapid Cycling and Atypical Forms.Cambridge University Press, Cambridge: pp 311-323.

Hantouche EG, Akiskal HS, Azorin JM, Chatenet-Duchene L, Lancrenon S (2006) Clinical and psychometric characterization of depression in mixed mania: a report from the French National Cohort of 1090 manic patients. J Affect Disord 96:225-232.

Harrington CA, English C (2010) Tolerability of paliperidone: a meta-analysis of randomized, controlled trials. Int Clin Psychopharmacol 25:334-341.

Himmelhoch JM, Garfinkel ME (1986) Sources of lithium resistance in mixed mania. Psychopharmacol Bull 22:613-620.

Houston JP, Tohen M, Degenhardt EK, Jamal HH, Liu LL, Ketter TA (2009) Olanzapinedivalproex combination versus divalproex monotherapy in the treatment of bipolar mixed episodes: a double-blind, placebo-controlled study. J Clin Psychiatry 70:1540-1547.

Huf W, Kalcher K, Pail G, Friedrich ME, Filzmoser P, Kasper S (2011) Meta-analysis: fact or fiction? How to interpret meta-analyses. World J Biol Psychiatry 12:188-200.

Jefferson JW (2010) A clinician's guide to monitoring kidney function in lithium-treated patients. J Clin Psychiatry 71:1153-1157.

Joas E, Karanti A, Song J, Goodwin GM, Lichtenstein P, Landen M (2017) Pharmacological treatment and risk of psychiatric hospital admission in bipolar disorder. Br J Psychiatry 210:197-202. 
Johnston AM, Eagles JM (1999) Lithium-associated clinical hypothyroidism. Prevalence and risk factors. Br J Psychiatry 175:336-339.

Jones MW (1998) Topiramate--safety and tolerability. Can J Neurol Sci 25:S13-S15.

Kanba S, Kawasaki H, Ishigooka J, Sakamoto K, Kinoshita T, Kuroki T (2014) A placebocontrolled, double-blind study of the efficacy and safety of aripiprazole for the treatment of acute manic or mixed episodes in Asian patients with bipolar I disorder (the AMAZE study). World J Biol Psychiatry 15:113-121.

Katagiri H, Takita Y, Tohen M, Higuchi T, Kanba S, Takahashi M (2012) Efficacy and safety of olanzapine in the treatment of Japanese patients with bipolar I disorder in a current manic or mixed episode: a randomized, double-blind, placebo- and haloperidol-controlled study. J Affect Disord 136:476-484.

Keck PE, Calabrese JR, McIntyre RS, McQuade RD, Carson WH, Eudicone JM, Carlson BX, Marcus RN, Sanchez R (2007) Aripiprazole monotherapy for maintenance therapy in bipolar I disorder: a 100-week, double-blind study versus placebo. J Clin Psychiatry 68:1480-1491.

Keck PE, Calabrese JR, McQuade RD, Carson WH, Carlson BX, Rollin LM, Marcus RN, Sanchez R (2006) A Randomized, Double-Blind, Placebo-Controlled 26-Week Trial of Aripiprazole in Recently Manic Patients With Bipolar I Disorder. J Clin Psychiatry 67:626-637.

Keck PE, Marcus R, Tourkodimitris S, Ali M, Liebeskind A, Saha A, Ingenito G (2003a) A placebo-controlled, double-blind study of the efficacy and safety of aripiprazole in patients with acute bipolar mania. Am J Psychiatry 160:1651-1658.

Keck PE, Orsulak PJ, Cutler AJ, Sanchez R, Torbeyns A, Marcus RN, McQuade RD, Carson WH (2009a) Aripiprazole monotherapy in the treatment of acute bipolar I mania: a randomized, double-blind, placebo- and lithium-controlled study. J Affect Disord 112:3649.

Keck PE, Versiani M, Potkin S, West SA, Giller E, Ice K (2003b) Ziprasidone in the treatment of acute bipolar mania: a three-week, placebo-controlled, double-blind, randomized trial. Am J Psychiatry 160:741-748.

Keck PE, Versiani M, Warrington L, Loebel AD, Horne RL (2009b) Long-term safety and efficacy of ziprasidone in subpopulations of patients with bipolar mania. J Clin Psychiatry 70:844-851.

Keller MB, Lavori PW, Coryell W, Endicott J, Mueller TI (1993) Bipolar I: a five-year prospective follow-up. J Nerv Ment Dis 181:238-245.

Kemp DE, Karayal ON, Calabrese JR, Sachs GS, Pappadopulos E, Ice KS, Siu CO, Vieta E (2012) Ziprasidone with adjunctive mood stabilizer in the maintenance treatment of bipolar I disorder: long-term changes in weight and metabolic profiles. Eur Neuropsychopharmacol 22:123-131.

Kessing LV, Gerds TA, Feldt-Rasmussen B, Andersen PK, Licht RW (2015) Use of Lithium and Anticonvulsants and the Rate of Chronic Kidney Disease: A Nationwide PopulationBased Study. JAMA Psychiatry 72:1182-1191. 
Kessing LV, Hellmund G, Andersen PK (2011a) An observational nationwide register based cohort study on lamotrigine versus lithium in bipolar disorder. J Psychopharmacol.

Kessing LV, Hellmund G, Geddes JR, Goodwin GM, Andersen PK (2011b) Valproate v. lithium in the treatment of bipolar disorder in clinical practice: observational nationwide register-based cohort study. Br J Psychiatry 199:57-63;Corrections in: Br.J Psychiatry 2011, 199 (4) 343.

Ketter TA, Kalali AH, Weisler RH (2004) A 6-month, multicenter, open-label evaluation of beaded, extended-release carbamazepine capsule monotherapy in bipolar disorder patients with manic or mixed episodes. J Clin Psychiatry 65:668-673.

Ketter TA, Sarma K, Silva R, Kroger H, Cucchiaro J, Loebel A (2016) Lurasidone in the long-term treatment of patients with Bipolar Disorder: A 24-week open-label extension study. Depress Anxiety 33:424-434.

Ketter TA, Winsberg ME, DeGolia SG, Dunai M, Tate DL, Strong CM (1998) Rapid efficacy of olanzapine augmentation in nonpsychotic bipolar mixed states. J Clin Psychiatry 59:8385.

Khanna S, Vieta E, Lyons B, Grossman F, Eerdekens M, Kramer M (2005) Risperidone in the treatment of acute mania: double-blind, placebo-controlled study. Br J Psychiatry 187:229234.

Koukopoulos A, Koukopoulos A (1999) Agitated depression as a mixed state and the problem of melancholia. Psychiatr Clin North Am 22:547-564.

Koukopoulos A, Sani G, Koukopoulos AE, Manfredi G, Pacchiarotti I, Girardi P (2007) Melancholia agitata and mixed depression. Acta Psychiatr Scand Suppl50-57.

Kraepelin E. 1899. Psychiatrie. Ein Lehrbuch für Studierende und Ärzte. 6 ed. Barth, Leipzig.

Lauer MS, Collins FS (2010) Using science to improve the nation's health system: NIH's commitment to comparative effectiveness research. JAMA 303:2182-2183.

Lee HJ, Son GH, Geum D (2013) Circadian rhythm hypotheses of mixed features, antidepressant treatment resistance, and manic switching in bipolar disorder. Psychiatry Investig 10:225-232.

Licht RW (2011) Lithium: Still a Major Option in the Management of Bipolar Disorder. CNS Neurosci Ther.

Loebel A, Cucchiaro J, Silva R, Kroger H, Hsu J, Sarma K, Sachs G (2014a) Lurasidone monotherapy in the treatment of bipolar I depression: a randomized, double-blind, placebocontrolled study. Am J Psychiatry 171:160-168.

Loebel A, Cucchiaro J, Silva R, Kroger H, Sarma K, Xu J, Calabrese JR (2014b) Lurasidone as adjunctive therapy with lithium or valproate for the treatment of bipolar I depression: a randomized, double-blind, placebo-controlled study. Am J Psychiatry 171:169-177.

Lundh A, Krogsboll LT, Gotzsche PC (2012) Sponsors' participation in conduct and reporting of industry trials: a descriptive study. Trials 13:146. doi: 10.1186/1745-6215-13-146.:14613. 
Malhi GS, Lampe L, Coulston CM, Tanious M, Bargh DM, Curran G, Kuiper S, Morgan H, Fritz K (2014) Mixed state discrimination: a DSM problem that wont go away? J Affect Disord 158:8-10.

Marcotte D (1998) Use of topiramate, a new anti-epileptic as a mood stabilizer. J Affect Disord 50:245-251.

Marcus R, Khan A, Rollin L, Morris B, Timko K, Carson W, Sanchez R (2011) Efficacy of aripiprazole adjunctive to lithium or valproate in the long-term treatment of patients with bipolar I disorder with an inadequate response to lithium or valproate monotherapy: a multicenter, double-blind, randomized study. Bipolar Disord 13:133-144.

Marneros A (2001a) Expanding the group of bipolar disorders. J Affect Disord 62:39-44.

Marneros A (2001b) Origin and development of concepts of bipolar mixed states. J Affect Disord 67:229-240.

Masi G, Mucci M, Millepiedi S (2002) Clozapine in adolescent inpatients with acute mania. J Child Adolesc Psychopharmacol 12:93-99.

McElroy SL, Keck PE, Pope HG, Hudson JI, Faedda GL, Swann AC (1992) Clinical and research implications of the diagnosis of dysphoric or mixed mania or hypomania. Am J Psychiatry 149:1633-1644.

McElroy SL, Soutullo CA, Keck PE, Jr., Kmetz GF (1997) A pilot trial of adjunctive gabapentin in the treatment of bipolar disorder. Ann Clin Psychiatry 9:99-103.

McIntyre R, Hirschfeld R, Alphs L, Cohen M, Macek T, Panagides J (2008) Randomized, placebo-controlled studies of asenapine in the treatment of acute mania in bipolar I disorder (Ares 1004/1005). J.Affect.Disord. 107 Suppl 1:56.

McIntyre RS, Cohen M, Zhao J, Alphs L, Macek TA, Panagides J (2010a) Asenapine for long-term treatment of bipolar disorder: a double-blind 40-week extension study. J Affect Disord 126:358-365.

McIntyre RS, Cohen M, Zhao J, Alphs L, Macek TA, Panagides J (2009) A 3-week, randomized, placebo-controlled trial of asenapine in the treatment of acute mania in bipolar mania and mixed states. Bipolar Disord 11:673-686.

McIntyre RS, Cohen M, Zhao J, Alphs L, Macek TA, Panagides J (2010b) Asenapine in the treatment of acute mania in bipolar I disorder: A randomized, double-blind, placebocontrolled trial. J Affect Disord 122:27-38.

McIntyre RS, Cucchiaro J, Pikalov A, Kroger H, Loebel A (2015) Lurasidone in the treatment of bipolar depression with mixed (subsyndromal hypomanic) features: post hoc analysis of a randomized placebo-controlled trial. J Clin Psychiatry 76:398-405.

McIntyre RS, McElroy SL, Eudicone JM, Forbes RA, Carlson BX, Baker RA (2011) A 52week, double-blind evaluation of the metabolic effects of aripiprazole and lithium in bipolar I disorder. Prim Care Companion CNS Disord 13:CC. 
McIntyre RS, Tohen M, Berk M, Zhao J, Weiller E (2013) DSM-5 mixed specifier for manic episodes: evaluating the effect of depressive features on severity and treatment outcome using asenapine clinical trial data. J Affect Disord 150:378-383.

Meador KJ, Baker GA, Browning N, Clayton-Smith J, Combs-Cantrell DT, Cohen M, Kalayjian LA, Kanner A, Liporace JD, Pennell PB, et al. (2009) Cognitive function at 3 years of age after fetal exposure to antiepileptic drugs. N Engl J Med 360:1597-1605.

Mech AW (2008) High-dose ziprasidone monotherapy in bipolar I disorder patients with depressed or mixed episodes. J Clin Psychopharmacol 28:240-241.

Medda P, Mauri M, Fratta S, Ciaponi B, Miniati M, Toni C, Dell'Osso L, Perugi G (2013) Long-term naturalistic follow-up of patients with bipolar depression and mixed state treated with electroconvulsive therapy. J ECT 29:179-188.

Medda P, Perugi G, Zanello S, Ciuffa M, Rizzato S, Cassano GB (2010) Comparative response to electroconvulsive therapy in medication-resistant bipolar I patients with depression and mixed state. J ECT 26:82-86.

Medda P, Toni C, Mariani MG, De Simone L, Mauri M, Perugi G (2015) Electroconvulsive therapy in 197 patients with a severe, drug-resistant bipolar mixed state: treatment outcome and predictors of response. J Clin Psychiatry 76:1168-1173.

Miura T, Noma H, Furukawa TA, Mitsuyasu H, Tanaka S, Stockton S, Salanti G, Motomura K, Shimano-Katsuki S, Leucht S, et al. (2014) Comparative efficacy and tolerability of pharmacological treatments in the maintenance treatment of bipolar disorder: a systematic review and network meta-analysis. Lancet Psychiatry 1:351-359.

Mokhber N, Lane CJ, Azarpazhooh MR, Salari E, Fayazi R, Shakeri MT, Young AH (2008) Anticonvulsant treatments of dysphoric mania: a trial of gabapentin, lamotrigine and carbamazepine in Iran. Neuropsychiatr Dis Treat 4:227-234.

Morrow J, Russell A, Guthrie E, Parsons L, Robertson I, Waddell R, Irwin B, McGivern RC, Morrison PJ, Craig J (2006) Malformation risks of antiepileptic drugs in pregnancy: a prospective study from the UK Epilepsy and Pregnancy Register. J Neurol Neurosurg Psychiatry 77:193-198.

Mortensen GL, Vinberg M, Lee MS, Balslev JM, Eberhard J (2015) Bipolar patients' quality of life in mixed states: a preliminary qualitative study. Psychopathology 48:192-201.

Muralidharan K, Ali M, Silveira LE, Bond DJ, Fountoulakis KN, Lam RW, Yatham LN (2013) Efficacy of second generation antipsychotics in treating acute mixed episodes in bipolar disorder: a meta-analysis of placebo-controlled trials. J Affect Disord 150:408-414.

Murru A, Manchia M, Tusconi M, Carpiniello B, Pacchiarotti I, Colom F, Vieta E (2016) Diagnostic reliability in schizoaffective disorder. Bipolar Disord 18:78-80.

Nasrallah HA, Newcomer JW (2004) Atypical antipsychotics and metabolic dysregulation: evaluating the risk/benefit equation and improving the standard of care. J Clin Psychopharmacol 24:S7-14.

Nguyen HT, Sharma V, McIntyre RS (2009) Teratogenesis associated with antibipolar agents. Adv Ther 26:281-294. 
Niedermier JA, Nasrallah HA (1998) Clinical correlates of response to valproate in geriatric inpatients. Ann Clin Psychiatry 10:165-168.

Nolen WA, Weisler RH (2013) The association of the effect of lithium in the maintenance treatment of bipolar disorder with lithium plasma levels: a post hoc analysis of a doubleblind study comparing switching to lithium or placebo in patients who responded to quetiapine (Trial 144). Bipolar Disord 15:100-109.

Ostergaard SD, Rothschild AJ, Bertelsen A, Mors O (2012) Rethinking the classification of mixed affective episodes in ICD-11. J Affect Disord 138:170-172.

Oxman AD, Guyatt GH (1992) A consumer's guide to subgroup analyses. Ann Intern Med 116:78-84.

Pacchiarotti I, Bond DJ, Baldessarini RJ, Nolen WA, Grunze H, Licht RW, Post RM, Berk M, Goodwin GM, Sachs GS, et al. (2013) The International Society for Bipolar Disorders (ISBD) task force report on antidepressant use in bipolar disorders. Am J Psychiatry 170:1249-1262.

Pacchiarotti I, Mazzarini L, Kotzalidis GD, Valenti M, Nivoli AM, Sani G, Torrent C, Murru A, Sanchez-Moreno J, Patrizi B, et al. (2011) Mania and depression. Mixed, not stirred. J Affect Disord 133:105-113.

Pae CU, Patkar AA, Gilmer W, Holtzman N, Thommi SB, Ghaemi SN (2012) Predictors of response to ziprasidone: results from a 6-week randomized double-blind, placebocontrolled trial for acute depressive mixed state. Pharmacopsychiatry 45:152-155.

Palma M, Ferreira B, Borja-Santos N, Trancas B, Monteiro C, Cardoso G (2016) Efficacy of Electroconvulsive Therapy in Bipolar Disorder with Mixed Features. Depress Res Treat 2016:8306071. doi: 10.1155/2016/8306071. Epub@2016 Jan 5.:8306071.

Patkar A, Gilmer W, Pae CU, Vohringer PA, Ziffra M, Pirok E, Mulligan M, Filkowski MM, Whitham EA, Holtzman NS, et al. (2012) A 6 week randomized double-blind placebocontrolled trial of ziprasidone for the acute depressive mixed state. PLoS One 7:e34757.

Perlis RH, Baker RW, Zarate CA, Jr., Brown EB, Schuh LM, Jamal HH, Tohen M (2006) Olanzapine versus risperidone in the treatment of manic or mixed States in bipolar I disorder: a randomized, double-blind trial. J Clin Psychiatry 67:1747-1753.

Perugi G, Akiskal HS, Micheli C, Toni C, Madaro D (2001) Clinical characterization of depressive mixed state in bipolar-I patients: Pisa-San Diego collaboration. J Affect Disord 67:105-114.

Perugi G, Angst J, Azorin JM, Bowden CL, Caciagli A, Mosolov S, Vieta E, Young AH (2015) Relationships between mixed features and borderline personality disorder in 2811 patients with major depressive episode. Acta Psychiatr Scand10.

Perugi G, Medda P, Reis J, Rizzato S, Giorgi MM, Mauri M (2013) Clinical subtypes of severe bipolar mixed states. J Affect Disord 151:1076-1082.

Perugi G, Micheli C, Akiskal HS, Madaro D, Socci C, Quilici C, Musetti L (2000) Polarity of the first episode, clinical characteristics, and course of manic depressive illness: a 
systematic retrospective investigation of 320 bipolar I patients. Compr Psychiatry 41:1318.

Perugi G, Toni C, Ruffolo G, Sartini S, Simonini E, Akiskal H (1999) Clinical experience using adjunctive gabapentin in treatment-resistant bipolar mixed states. Pharmacopsychiatry 32:136-141.

Pigott K, Galizia I, Vasudev K, Watson S, Geddes J, Young AH (2016) Topiramate for acute affective episodes in bipolar disorder in adults. Cochrane Database Syst Rev 9:CD003384.:CD003384.

Pikalov A, Tsai J, Mao Y, Silva R, Cucchiaro J, Loebel A (2017) Long-term use of lurasidone in patients with bipolar disorder: safety and effectiveness over 2 years of treatment. Int $\mathrm{J}$ Bipolar Disord 5:9-0075.

Post RM, Rubinow DR, Uhde TW, Roy-Byrne PP, Linnoila M, Rosoff A, Cowdry R (1989) Dysphoric mania. Clinical and biological correlates. Arch Gen Psychiatry 46:353-358.

Potkin SG, Keck PE, Jr., Segal S, Ice K, English P (2005) Ziprasidone in Acute Bipolar Mania: A 21-Day Randomized, Double-Blind, Placebo-Controlled Replication Trial. J Clin Psychopharmacol 25:301-310.

Preti A, Vrublevska J, Veroniki AA, Huedo-Medina TB, Fountoulakis KN (2016) Prevalence, impact and treatment of generalised anxiety disorder in bipolar disorder: a systematic review and meta-analysis. Evid Based Ment Health 19:73-81.

Prien RF, Himmelhoch JM, Kupfer DJ (1988) Treatment of mixed mania. J Affect Disord 15:9-15.

Radua J, Grunze H, Amann BL (2017) Meta-Analysis of the Risk of Subsequent Mood Episodes in Bipolar Disorder. Psychother Psychosom 86:90-98.

Reinares M, Bonnin CM, Hidalgo-Mazzei D, Undurraga J, Mur M, Nieto E, Saez C, Vieta E (2015) Making sense of DSM-5 mania with depressive features. Aust N Z J Psychiatry 49:540-549.

Sachs G, Sanchez R, Marcus R, Stock E, McQuade R, Carson W, bou-Gharbia N, Impellizzeri C, Kaplita S, Rollin L, et al. (2006) Aripiprazole in the treatment of acute manic or mixed episodes in patients with bipolar I disorder: a 3-week placebo-controlled study. J Psychopharmacol 20:536-546.

Sachs GS, Greenberg WM, Starace A, Lu K, Ruth A, Laszlovszky I, Nemeth G, Durgam S (2015) Cariprazine in the treatment of acute mania in bipolar I disorder: a double-blind, placebo-controlled, phase III trial. J Affect Disord 174:296-302.

Sachs GS, Grossman F, Ghaemi SN, Okamoto A, Bowden CL (2002) Combination of a mood stabilizer with risperidone or haloperidol for treatment of acute mania: a double-blind, placebo-controlled comparison of efficacy and safety. Am J Psychiatry 159:1146-1154.

Sajatovic M, Al JR, Gildengers A, Greenberg RL, Tenhave T, Bruce ML, Mulsant B, Young RC (2011) Depression symptom ratings in geriatric patients with bipolar mania. Int J Geriatr Psychiatry 26:1201-1208. 
Salvatore P, Baldessarini RJ, Centorrino F, Egli S, Albert M, Gerhard A, Maggini C (2002) Weygandt's On the Mixed States of Manic-Depressive Insanity: a translation and commentary on its significance in the evolution of the concept of bipolar disorder. Harv Rev Psychiatry 10:255-275.

Sato T, Bottlender R, Sievas M, Schroter A, Hecht S, Moller HJ (2003) Long-term interepisode stability of syndromes underlying mania. Acta Psychiatr Scand 108:310-313.

Sawyer L, Azorin JM, Chang S, Rinciog C, Guiraud-Diawara A, Marre C, Hansen K (2014) Cost-effectiveness of asenapine in the treatment of bipolar I disorder patients with mixed episodes. J Med Econ 17:508-519.

Schaffer A, Isometsa ET, Tondo L, Moreno DH, Sinyor M, Kessing LV, Turecki G, Weizman A, Azorin JM, Ha K, et al. (2015) Epidemiology, neurobiology and pharmacological interventions related to suicide deaths and suicide attempts in bipolar disorder: Part I of a report of the International Society for Bipolar Disorders Task Force on Suicide in Bipolar Disorder. Aust N Z J Psychiatry 49:785-802.

Schaffer CB, Schaffer LC, Nordahl TE, Stark NM, Gohring CE (2016) An Open Trial of Lurasidone as an Acute and Maintenance Adjunctive Treatment for Outpatients With Treatment-Resistant Bipolar Disorder. J Clin Psychopharmacol 36:88-89.

Secunda SK, Swann A, Katz MM, Koslow SH, Croughan J, Chang S (1987) Diagnosis and treatment of mixed mania. Am J Psychiatry 144:96-98.

Sedler MJ (1983) Falret's discovery: the origin of the concept of bipolar affective illness. Translated by M. J. Sedler and Eric C. Dessain. Am J Psychiatry 140:1127-1133.

Seemüller F, Forsthoff A, Dittmann S, Born C, Bernhard B, Severus WE, Grunze H (2005) The safety and tolerability of atypical antipsychotics in bipolar disorder. Expert Opin Drug Saf 4:849-868.

Sharma V, Pistor L (1999) Treatment of bipolar mixed state with olanzapine. J Psychiatry Neurosci 24:40-44.

Shim IH, Woo YS, Bahk WM (2015) Prevalence rates and clinical implications of bipolar disorder "with mixed features" as defined by DSM-5. J Affect Disord 173:120-5. doi: 10.1016/j.jad.2014.10.061. Epub@2014 Nov 10.:120-125.

Shorter D, Healy D. 2007. Shock Therapy: A History of Electroconvulsive Therapy in Mental Illness. London, UK: Rutgers University Press; 2007. Rutgers University Press, London.

Sokolski KN, Green C, Maris DE, DeMet EM (1999) Gabapentin as an adjunct to standard mood stabilizers in outpatients with mixed bipolar symptomatology. Ann Clin Psychiatry 11:217-222.

Spina E, Pisani F, Perucca E (1996) Clinically significant pharmacokinetic drug interactions with carbamazepine. An update. Clin Pharmacokinet 31:198-214.

Spitzer RL, Endicott J, Robbins E. 1975. Research Diagnostic criteria (RDC) for a selected group of functional disorders. 2 ed. New York Psychiatric Institute, New York. 
Stahl S, Lombardo I, Loebel A, Mandel FS (2010) Efficacy of ziprasidone in dysphoric mania: pooled analysis of two double-blind studies. J Affect Disord 122:39-45.

Stahl SM, Morrissette DA, Faedda G, Fava M, Goldberg JF, Keck PE, Lee Y, Malhi G, Marangoni C, McElroy SL, et al. (2017) Guidelines for the recognition and management of mixed depression. CNS Spectrums 22:203-219.

Strakowski SM, McElroy SL, Keck PE, West SA (1996) Suicidality among patients with mixed and manic bipolar disorder. Am J Psychiatry 153:674-676.

Strejilevich SA, Martino DJ, Marengo E, Igoa A, Fassi G, Whitham EA, Ghaemi SN (2011) Long-term worsening of bipolar disorder related with frequency of antidepressant exposure. Ann Clin Psychiatry 23:186-192.

Strömgren LS (1988) Electroconvulsive Therapy in Aarhus, Denmark, in 1984: Its Application in Nondepressive Disorders. Convuls Ther 4:306-313.

Suppes T, Eudicone J, McQuade R, Pikalov A, III, Carlson B (2008) Efficacy and safety of aripiprazole in subpopulations with acute manic or mixed episodes of bipolar I disorder. $\mathrm{J}$ Affect Disord 107:145-154.

Suppes T, Ketter TA, Gwizdowski IS, Dennehy EB, Hill SJ, Fischer EG, Snow DE, Gonzalez R, Sureddi S, Shivakumar G, et al. (2013) First controlled treatment trial of bipolar II hypomania with mixed symptoms: quetiapine versus placebo. J Affect Disord 150:37-43.

Suppes T, Kroger H, Pikalov A, Loebel A (2016a) Lurasidone adjunctive with lithium or valproate for bipolar depression: A placebo-controlled trial utilizing prospective and retrospective enrolment cohorts. J Psychiatr Res 78:86-93.

Suppes T, McElroy SL, Gilbert J, Dessain EC, Cole JO (1992) Clozapine in the treatment of dysphoric mania. Biol Psychiatry 32:270-280.

Suppes T, Mintz J, McElroy SL, Altshuler LL, Kupka RW, Frye MA, Keck PE, Jr., Nolen WA, Leverich GS, Grunze H, et al. (2005) Mixed hypomania in 908 patients with bipolar disorder evaluated prospectively in the Stanley Foundation Bipolar Treatment Network: a sex-specific phenomenon. Arch Gen Psychiatry 62:1089-1096.

Suppes T, Silva R, Cucchiaro J, Mao Y, Targum S, Streicher C, Pikalov A, Loebel A (2016b) Lurasidone for the Treatment of Major Depressive Disorder With Mixed Features: A Randomized, Double-Blind, Placebo-Controlled Study. Am J Psychiatry 173:400-407.

Suppes T, Vieta E, Liu S, Brecher M, Paulsson B (2009) Maintenance treatment for patients with bipolar I disorder: results from a north american study of quetiapine in combination with lithium or divalproex (trial 127). Am J Psychiatry 166:476-488.

Swann AC (2000) Depression, mania, and feeling bad: the role of dysphoria in mixed states. Bipolar Disord 2:325-327.

Swann AC, Bowden CL, Calabrese J, Dilsaver SC, Morris DD (2002) Pattern of response to divalproex, lithium, or placebo in four naturalistic subtypes of mania. Neuropsychopharmacology 26:530-536. 
Swann AC, Bowden CL, Morris D, Calabrese JR, Petty F, Small J, Dilsaver SC, Davis JM (1997) Depression during mania. Treatment response to lithium or divalproex. Arch Gen Psychiatry 54:37-42.

Swann AC, Secunda SK, Katz MM, Koslow SH, Maas JW, Chang S, Robins E (1986) Lithium treatment of mania: clinical characteristics, specificity of symptom change, and outcome. Psychiatry Res 18:127-141.

Szegedi A, Calabrese JR, Stet L, Mackle M, Zhao J, Panagides J (2012) Asenapine as adjunctive treatment for acute mania associated with bipolar disorder: results of a 12-week core study and 40-week extension. J Clin Psychopharmacol 32:46-55.

Szegedi A, Zhao J, McIntyre RS (2013) Early improvement as a predictor of acute treatment outcome in manic or mixed episodes in bipolar-1 disorder: a pooled, post hoc analysis from the asenapine development program. J Affect Disord 150:745-752.

Szegedi A, Zhao J, van WA, Nations KR, Mackle M, Panagides J (2011) Effects of asenapine on depressive symptoms in patients with bipolar I disorder experiencing acute manic or mixed episodes: a post hoc analysis of two 3-week clinical trials. BMC Psychiatry 11:101.

Tang CS, Yeh CB, Huang YS, Wang LJ, Chou WJ, Chou MC, Chen CK (2010) Long-term effectiveness of aripiprazole in adolescents and young adults with bipolar disorder: A naturalistic study. Int J Psychiatry Clin Pract 14:252-256.

Targum SD, Suppes T, Pendergrass JC, Lee S, Silva R, Cucchiaro J, Loebel A (2016) Major depressive disorder with subthreshold hypomania (mixed features): Clinical characteristics of patients entered in a multiregional, placebo-controlled study. Prog Neuropsychopharmacol Biol Psychiatry 68:9-14.

Taylor DM, Cornelius V, Smith L, Young AH (2014) Comparative efficacy and acceptability of drug treatments for bipolar depression: a multiple-treatments meta-analysis. Acta Psychiatr Scand 130:452-469.

Tohen M, Calabrese JR, Sachs GS, Banov MD, Detke HC, Risser R, Baker RW, Chou JC, Bowden CL (2006) Randomized, placebo-controlled trial of olanzapine as maintenance therapy in patients with bipolar I disorder responding to acute treatment with olanzapine. Am J Psychiatry 163:247-256.

Tohen M, Chengappa KN, Suppes T, Baker RW, Zarate CA, Bowden CL, Sachs GS, Kupfer DJ, Ghaemi SN, Feldman PD, et al. (2004) Relapse prevention in bipolar I disorder: 18month comparison of olanzapine plus mood stabiliser v. mood stabiliser alone. Br J Psychiatry 184:337-345.

Tohen M, Chengappa KN, Suppes T, Zarate CA, Calabrese JR, Bowden CL, Sachs GS, Kupfer DJ, Baker RW, Risser RC, et al. (2002) Efficacy of olanzapine in combination with valproate or lithium in the treatment of mania in patients partially nonresponsive to valproate or lithium monotherapy. Arch Gen Psychiatry 59:62-69.

Tohen M, Goldberg JF, Gonzalez-Pinto Arrillaga AM, Azorin JM, Vieta E, Hardy-Bayle MC, Lawson WB, Emsley RA, Zhang F, Baker RW, et al. (2003a) A 12-week, double-blind comparison of olanzapine vs haloperidol in the treatment of acute mania. Arch Gen Psychiatry 60:1218-1226. 
Tohen M, Greenfield SF, Weiss RD, Zarate CA, Jr., Vagge LM (1998) The effect of comorbid substance use disorders on the course of bipolar disorder: a review. Harv Rev Psychiatry 6:133-141.

Tohen M, Greil W, Calabrese JR, Sachs GS, Yatham LN, Oerlinghausen BM, Koukopoulos A, Cassano GB, Grunze H, Licht RW, et al. (2005) Olanzapine versus lithium in the maintenance treatment of bipolar disorder: a 12-month, randomized, double-blind, controlled clinical trial. Am J Psychiatry 162:1281-1290.

Tohen M, Jacobs TG, Grundy SL, McElroy SL, Banov MC, Janicak PG, Sanger T, Risser R, Zhang F, Toma V, et al. (2000) Efficacy of olanzapine in acute bipolar mania: A doubleblind, placebo-controlled study. Arch Gen Psychiatry 57:841-849.

Tohen M, Kanba S, McIntyre RS, Fujikoshi S, Katagiri H (2014a) Efficacy of olanzapine monotherapy in the treatment of bipolar depression with mixed features. J Affect Disord 164:57-62.

Tohen M, Kryzhanovskaya L, Carlson G, DelBello M, Wozniak J, Kowatch R, Wagner K, Findling R, Lin D, Robertson-Plouch C, et al. (2007) Olanzapine versus placebo in the treatment of adolescents with bipolar mania. Am J Psychiatry 164:1547-1556.

Tohen M, McDonnell DP, Case M, Kanba S, Ha K, Fang YR, Katagiri H, Gomez JC (2012) Randomised, double-blind, placebo-controlled study of olanzapine in patients with bipolar I depression. Br J Psychiatry 201:376-382.

Tohen M, McIntyre RS, Kanba S, Fujikoshi S, Katagiri H (2014b) Efficacy of olanzapine in the treatment of bipolar mania with mixed features defined by DSM-5. J Affect Disord 168:136-141.

Tohen M, Mintz J, Bowden CL (2016) Analysis of bipolar maintenance treatment with lithium versus olanzapine utilizing Multi-state Outcome Analysis of Treatments (MOAT). Bipolar Disord 18:282-287.

Tohen M, Sanger TM, McElroy SL, Tollefson GD, Chengappa KN, Daniel DG, Petty F, Centorrino F, Wang R, Grundy SL, et al. (1999) Olanzapine versus placebo in the treatment of acute mania. Olanzapine HGEH Study Group. Am J Psychiatry 156:702-709.

Tohen M, Sutton VK, Calabrese JR, Sachs GS, Bowden CL (2009) Maintenance of response following stabilization of mixed index episodes with olanzapine monotherapy in a randomized, double-blind, placebo-controlled study of bipolar 1 disorder. J Affect Disord 116:43-50.

Tohen M, Vieta E, Calabrese J, Ketter TA, Sachs G, Bowden C, Mitchell PB, Centorrino F, Risser R, Baker RW, et al. (2003b) Efficacy of olanzapine and olanzapine-fluoxetine combination in the treatment of bipolar I depression. Arch Gen Psychiatry 60:1079-1088.

Tohen M, Vieta E, Goodwin GM, Sun B, Amsterdam JD, Banov M, Shekhar A, Aaronson ST, Bardenstein L, Grecu-Gabos I, et al. (2008) Olanzapine versus divalproex versus placebo in the treatment of mild to moderate mania: a randomized, 12-week, double-blind study. J Clin Psychiatry 69:1776-1789.

Tredget J, Kirov A, Kirov G (2010) Effects of chronic lithium treatment on renal function. J Affect Disord 126:436-440. 
Tundo A, Musetti L, Benedetti A, Berti B, Massimetti G, Dell'Osso L (2015) Onset polarity and illness course in bipolar I and II disorders: The predictive role of broadly defined mixed states. Compr Psychiatry 63:15-21.

Tural U, Onder E (2010) Clinical and pharmacologic risk factors for neuroleptic malignant syndrome and their association with death. Psychiatry Clin Neurosci 64:79-87.

Turner EH, Matthews AM, Linardatos E, Tell RA, Rosenthal R (2008) Selective publication of antidepressant trials and its influence on apparent efficacy. N Engl J Med 358:252-260.

Valenti M, Benabarre A, Garcia-Amador M, Molina O, Bernardo M, Vieta E (2008) Electroconvulsive therapy in the treatment of mixed states in bipolar disorder. Eur Psychiatry 23:53-56.

Valenti M, Pacchiarotti I, Rosa AR, Bonnin CM, Popovic D, Nivoli AM, Murru A, Grande I, Colom F, Vieta E (2011) Bipolar mixed episodes and antidepressants: a cohort study of bipolar I disorder patients. Bipolar Disord 13:145-154.

Van Amelsvoort T, Bakshi R, Devaux CB, Schwabe S (1994) Hyponatremia associated with carbamazepine and oxcarbazepine therapy: a review. Epilepsia 35:181-188.

Vanelle JM, Loo H, Galinowski A, de Carvalho W, Bourdel MC, Brochier P, Bouvet O, Brochier T, Olie JP (1994) Maintenance ECT in intractable manic-depressive disorders. Convuls Ther 10:195-205.

Vieta E (2016) DSM-5.1. Acta Psychiatr Scand 134:187-188.

Vieta E, Bourin M, Sanchez R, Marcus R, Stock E, McQuade R, Carson W, Abou-Gharbia N, Swanink R, Iwamoto T (2005) Effectiveness of aripiprazole v. haloperidol in acute bipolar mania: double-blind, randomised, comparative 12-week trial. Br J Psychiatry 187:235-242.

Vieta E, Durgam S, Lu K, Laszlovszky I, Patel M, Earley W (2017) Efficacy of cariprazine in subgroups of bipolar patients with manic episodes, mixed episodes, and with or without psychotic symptoms . Eur Neuropsychopharmacol.

Vieta E, Durgam S, Lu K, Ruth A, Debelle M, Zukin S (2015) Effect of cariprazine across the symptoms of mania in bipolar I disorder: Analyses of pooled data from phase II/III trials. Eur Neuropsychopharmacol 25:1882-1891.

Vieta E, Gasto C, Escobar R (1995) Treatment of dysphoric mania with risperidone. Int J Neuropsychopharmacol 10:491-492.

Vieta E, Grunze H, Azorin JM, Fagiolini A (2014) Phenomenology of manic episodes according to the presence or absence of depressive features as defined in DSM-5: Results from the IMPACT self-reported online survey. J Affect Disord 156:206-213.

Vieta E, Martinez-Aran A, Nieto E, Colom F, Reinares M, Benabarre A, Gasto C (2000) Adjunctive gabapentin treatment of bipolar disorder. Eur Psychiatry 15:433-437.

Vieta E, Morralla C (2010) Prevalence of mixed mania using 3 definitions. J Affect Disord 125:61-73. 
Vieta E, Nuamah IF, Lim P, Yuen EC, Palumbo JM, Hough DW, Berwaerts J (2010) A randomized, placebo- and active-controlled study of paliperidone extended release for the treatment of acute manic and mixed episodes of bipolar I disorder. Bipolar Disord 12:230243.

Vieta E, Suppes T, Eggens I, Persson I, Paulsson B, Brecher M (2008a) Efficacy and safety of quetiapine in combination with lithium or divalproex for maintenance of patients with bipolar I disorder (international trial 126). J Affect Disord 109:251-263.

Vieta E, Suppes T, Ekholm B, Udd M, Gustafsson U (2012) Long-term efficacy of quetiapine in combination with lithium or divalproex on mixed symptoms in bipolar I disorder. $\mathrm{J}$ Affect Disord 142:36-44.

Vieta E, T'joen C, McQuade RD, Carson WH, Jr., Marcus RN, Sanchez R, Owen R, Nameche L (2008b) Efficacy of adjunctive aripiprazole to either valproate or lithium in bipolar mania patients partially nonresponsive to valproate/lithium monotherapy: a placebocontrolled study. Am J Psychiatry 165:1316-1325.

Vieta E, Torrent C, Garcia-Ribas G, Gilabert A, Garcia-Pares G, Rodriguez A, Cadevall J, Garcia-Castrillon J, Lusilla P, Arrufat F (2002) Use of topiramate in treatment-resistant bipolar spectrum disorders. J Clin Psychopharmacol 22:431-435.

Vieta E, Valenti M (2013) Mixed states in DSM-5: implications for clinical care, education, and research. J Affect Disord 148:28-36.

Vita A, De Peri L, Siracusano A, Sacchetti E (2013) Efficacy and tolerability of asenapine for acute mania in bipolar I disorder: meta-analyses of randomized-controlled trials. Int Clin Psychopharmacol 28:219-227.

Wehr TA, Goodwin FK (1987) Can antidepressants cause mania and worsen the course of affective illness? Am J Psychiatry 144:1403-1411.

Weibel S, Bertschy G (2016) Depression mixte et DSM-5 : mise au point critique. Encephale 42:90-98.

Weisler RH, Hirschfeld R, Cutler AJ, Gazda T, Ketter TA, Keck PE, Swann A, Kalali A (2006) Extended-release carbamazepine capsules as monotherapy in bipolar disorder : pooled results from two randomised, double-blind, placebo-controlled trials. CNS Drugs 20:219-231.

Weisler RH, Kalali AH, Ketter TA (2004) A multicenter, randomized, double-blind, placebocontrolled trial of extended-release carbamazepine capsules as monotherapy for bipolar disorder patients with manic or mixed episodes. J Clin Psychiatry 65:478-484.

Weisler RH, Keck PE, Jr., Swann AC, Cutler AJ, Ketter TA, Kalali AH (2005) Extendedrelease carbamazepine capsules as monotherapy for acute mania in bipolar disorder: a multicenter, randomized, double-blind, placebo-controlled trial. J Clin Psychiatry 66:323330.

Weisler RH, Nolen WA, Neijber A, Hellqvist A, Paulsson B (2011) Continuation of quetiapine versus switching to placebo or lithium for maintenance treatment of bipolar I disorder (Trial 144: a randomized controlled study). J Clin Psychiatry 72:1452-1464. 
Weygandt W. 1899. Über die Mischzustände des manisch-depressiven Irreseins. Habilitationsschrift. JF Lehmann, München.

Whittier MC, West SA, Galli VB, Raute NJ (1995) Valproic acid for dysphoric mania in a mentally retarded adolescent. J Clin Psychiatry 56:590-591.

Winokur G, Clayton PJ, Reich T. 1969. Manic Depressive illness. C.V.Mosby, St.Louis.

Wisner KL, Peindl KS, Perel JM, Hanusa BH, Piontek CM, Baab S (2002) Verapamil treatment for women with bipolar disorder. Biol Psychiatry 51:745-752.

Woo YS, Bahk WM, Chung MY, Kim DH, Yoon BH, Lee JH, Ahn YM, Chung SK, Kim JG, Lee KH, et al. (2011) Aripiprazole plus divalproex for recently manic or mixed patients with bipolar I disorder: a 6-month, randomized, placebo-controlled, double-blind maintenance trial. Hum psychopharmacol 26:543-553.

Woo YS, Bahk WM, Jon DI, Chung SK, Lee SY, Ahn YM, Pae CU, Cho HS, Kim JG, Hwang TY, et al. (2010) Risperidone in the treatment of mixed state bipolar patients: results from a 24-week, multicenter, open-label study in Korea. Psychiatry Clin Neurosci 64:28-37.

World Health Organization. 1992. The ICD-10 Classification of Mental and behavioural Disorders. Clinical Descriptions and Diagnostic Guidelines. WHO, Geneva.

Wu HE, Okusaga OO (2015) Antipsychotic medication-induced dysphoria: its meaning, association with typical vs. atypical medications and impact on adherence. Psychiatr Q 86:199-205.

Yatham LN, Fountoulakis KN, Rahman Z, Ammerman D, Fyans P, Marler SV, Baker RA, Carlson BX (2013) Efficacy of aripiprazole versus placebo as adjuncts to lithium or valproate in relapse prevention of manic or mixed episodes in bipolar I patients stratified by index manic or mixed episode. J Affect Disord 147:365-372.

Yatham LN, Grossman F, Augustyns I, Vieta E, Ravindran A (2003) Mood stabilisers plus risperidone or placebo in the treatment of acute mania. International, double-blind, randomised controlled trial. Br J Psychiatry 182:141-147.

Yatham LN, Vieta E, Goodwin GM, Bourin M, de BC, Laredo J, Calabrese J (2016) Agomelatine or placebo as adjunctive therapy to a mood stabiliser in bipolar I depression: randomised double-blind placebo-controlled trial. Br J Psychiatry 208:78-86.

Yildiz A, Nikodem M, Vieta E, Correll CU, Baldessarini RJ (2015) A network meta-analysis on comparative efficacy and all-cause discontinuation of antimanic treatments in acute bipolar mania. Psychol Med 45:299-317.

Young AH, Altamura AC, Gonzalez-Pinto AM, Millet B, Wiedemann K (2013) Use of asenapine in clinical practice for the management of bipolar mania. J Psychopharmacol 27:3-13.

Young AH, Eberhard J (2015) Evaluating depressive symptoms in mania: a naturalistic study of patients with bipolar disorder. Neuropsychiatr Dis Treat 11:1137-1143. 
Young AH, Oren DA, Lowy A, McQuade RD, Marcus RN, Carson WH, Spiller NH, Torbeyns AF, Sanchez R (2009) Aripiprazole monotherapy in acute mania: 12-week randomised placebo- and haloperidol-controlled study. Br J Psychiatry 194:40-48.

Zarate CA, Rothschild A, Fletcher KE, Madrid A, Zapatel J (2000) Clinical predictors of acute response with quetiapine in psychotic mood disorders. J Clin Psychiatry 61:185-189.

Zeeuws D, De RK, De RR, De BM, Baeken C, Vanderbruggen N (2011) Intensive highfrequency repetitive transcranial magnetic stimulation treatment in an electroconvulsive shock therapy-resistant bipolar I patient with mixed episode. Brain Stimul 4:46-49.

Zeeuws D, Santermans L, Baeken C, Vanderbruggen N (2010a) Intensive rTMS applications in difficult to treat psychiatric patients: some cases. Psychiatr Danub 22 Suppl 1:S1356.:S135-S136.

Zeeuws D, Santermans L, Baeken C, Vanderbruggen N (2010b) Combative HF-rTMS treatment, for a bipolar I patient, following unsuccessful ECT. Psychiatr Danub 22 Suppl 1:S164.:S164.

Zimbroff DL, Marcus RN, Manos G, Stock E, McQuade RD, Auby P, Oren DA (2007) Management of acute agitation in patients with bipolar disorder: efficacy and safety of intramuscular aripiprazole. J Clin Psychopharmacol 27:171-176.

Zullino D, Baumann P (1999) Olanzapine for mixed episodes of bipolar disorder. J Psychopharmacol 13:198. 